\title{
Development of new protocols to evaluate the transverse loading of mine ventilation stoppings
}

Thomas M. Barczak

West Virginia University

Follow this and additional works at: https://researchrepository.wvu.edu/etd

\section{Recommended Citation}

Barczak, Thomas M., "Development of new protocols to evaluate the transverse loading of mine ventilation stoppings" (2004). Graduate Theses, Dissertations, and Problem Reports. 1528.

https://researchrepository.wvu.edu/etd/1528

This Thesis is protected by copyright and/or related rights. It has been brought to you by the The Research Repository @ WVU with permission from the rights-holder(s). You are free to use this Thesis in any way that is permitted by the copyright and related rights legislation that applies to your use. For other uses you must obtain permission from the rights-holder(s) directly, unless additional rights are indicated by a Creative Commons license in the record and/ or on the work itself. This Thesis has been accepted for inclusion in WVU Graduate Theses, Dissertations, and Problem Reports collection by an authorized administrator of The Research Repository @ WVU. For more information, please contact researchrepository@mail.wvu.edu. 


\title{
DEVELOPMENT OF NEW PROTOCOLS TO EVALUATE THE TRANSVERSE LOADING OF MINE VENTILATION STOPPINGS
}

\author{
by \\ Thomas M. Barczak \\ Thesis submitted to the College of Engineering and Mineral Resources \\ West Virginia University \\ In partial fulfillment of the requirements for the degree of \\ Master of Science \\ in \\ Mining Engineering \\ Syd S. Peng, Ph.D., Chair \\ Keith A. Heasley, Ph.D. \\ Yi Luo, Ph.D. \\ Department of Mining Engineering \\ Morgantown, West Virginia \\ 2004
}

Keywords: stoppings, transverse load, ventilation

Copyright 2004 Thomas M. Barczak 


\title{
Development of New Protocols to Evaluate the Transverse Loading of Mine Ventilation Stoppings
}

by

Thomas M. Barczak

\begin{abstract}
The transverse loading requirement for stoppings as specified in the current Code of Federal Regulations is 39 psf. This measure is based on physical testing of a freestanding wall in accordance with ASTM E 72 specifications, where the dominant parameter is the tensile strength of the sealant. A new protocol based on rigid arching is proposed to determine the true transverse load capacity of stoppings. Arching is achieved by the restraint of the stopping against the mine roof and floor, whereby compressive forces are developed within the wall. A laboratory procedure using the NIOSH Mine Roof Simulator (MRS) to simulate rigid-arching of stoppings was developed and verified through full-scale in-mine tests. The rigid arching tests have demonstrated transverse load capacities more than an order of magnitude higher than the current 39-psf requirement. More importantly, the load capability is dependent on the physical properties of the block and not the sealant.
\end{abstract}




\section{DEDICATION}

I dedicate this thesis to my God Father, John Waddella, who realized the value of an education and encouraged me during my high school years to go on to college. He was and always will be my "best buddy". 


\section{ACKNOWLEDGEMENTS}

The author wishes to thank those who have contributed to the preparation and completion of this thesis. A debt of gratitude is owed to Dr. Syd S. Peng for encouraging me to continue my education and guidance in my studies at West Virginia University. Special appreciation is also given to the members of my advisory and examination committee: Dr. Keith Heasley and Dr. Yi Luo for their guidance and supervision.

The author also wishes to extend his deepest gratitude to Mr. David F. Gearhart and Mr. Timothy J. Batchler. Dave has been a colleague for the past 20 years and we have worked together on numerous projects during that time. Dave has been instrumental in conducting tests in both the Mine Roof Simulator and Experimental Coal Mine in support of this thesis. I also thank Dave for imparting his overall experience in testing of largescale structures and insights into the procedures developed for this study. Tim also contributed to this effort by operating the Mine Roof Simulator and processing raw data for many of the experiments that were done in support of this thesis. I would also like to acknowledge Lisa Burke, a colleague at NIOSH who recently completed her Masters Thesis at Virginia Tech on numerical modeling of stopping walls for axial loading induced by roof-to-floor closure of the mine opening. Lisa and I shared many discussions regarding the mechanics of the loading behavior of a stopping wall, which helped me tremendously in pursuing my goals in this thesis.

I must also express my gratitude to Dr. Gerald L. Finfinger and Dr. Stephen C. Tadolini, two old friends and long time colleagues, who after twenty years or prodding finally convinced me to do this.

I would also like to thank my fellow students, who accepted me as one of them even though I was nearly 30 years older than most of them. I must also give my sincerest thanks to Karen Centofanti, who was a tremendous help to this "non traditional" student,

who was lost most of the time in knowing where to go, who to see, and what to do in the administrative requirements of my schooling. 
Finally, I express my deepest appreciation to my family, my wife Peggy and daughters Sarah and Nora, for putting up with me while I was studying at home in the evenings and on weekends and for their overall support in allowing me to achieve this goal at their expense. 
Page

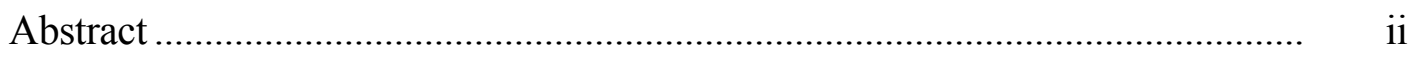

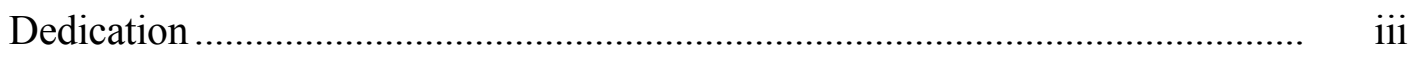

Acknowledgements ......................................................................................... iv

Table of contents..................................................................................... vi

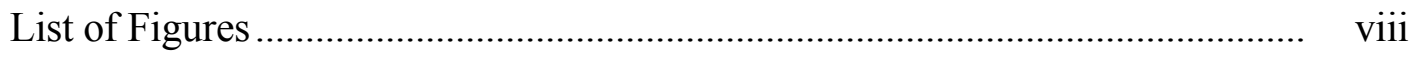

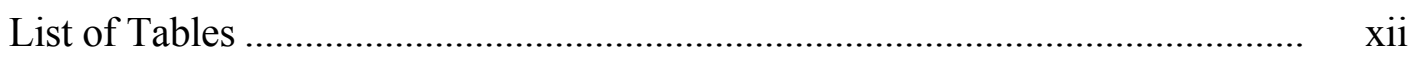

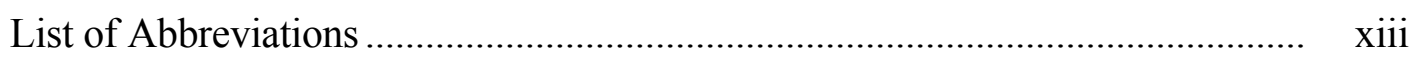

CHAPTER 1 - INTRODUCTION ………………………………………….....

CHAPTER 2 - REVIEW OF LITERATURE ................................................ 3

CHAPTER 3 - CURRENT CFR CRITERIA FOR STOPPINGS ..................... 7

3.1 ASTM E 72 Test Specifications ……………............................... 7

3.2 Inadequacies of Current CFR Specifications ................................ 10

3.3 Evaluation of Sealant Tensile Strength Characteristics ................. 11

3.4 Comparisons to Conventional Masonry Design Practices .............. 18

CHAPTER 4 - RIGID ARCH LOADING MECHANISM …………….......... 21

4.1 Physical Description of Arching .................................................. 21

4.2 Theoretical Assessment of Rigid Arching..................................... 23

$4.3 \quad$ Transverse Load Design Equation ............................................... 26

4.4 Implications of Rigid Arching to Stopping Design........................ 27

CHAPTER 5 - SIMULATING RIGID ARCHING THROUGH BIAXIAL

LOADING IN THE MINE ROOF SIMULATOR.................. 29

5.1 Description of the Mine Roof Simulator ......................................... 29

5.2 Test Protocol for Simulating Rigid Arching ……......................... 31

5.3 Transverse Load Determinations from MRS Half-Wall Testing.. 35

5.4 Comparison to Rigid Arching Theoretical Design ...................... 41

CHAPTER 6 - FULL-SCALE LOAD VERIFICATION TESTING................ 46

6.1 NIOSH PRL Experimental Coal Mine Tests ................................ 46 
6.1.1 Air Pressure Chamber ...................................................... 46

6.1.2 Test Results and Comparisons to Half-Wall MRS Tests.... 49

6.2 NIOSH Lake Lynn Laboratory Tests .............................................. 56

6.2.1 Explosive Charge Loading ………………...................... 57

6.2.2 Test Results and Comparisons to Half-Wall MRS Tests .. 58

SUMMARY AND CONCLUSIONS ........................................................... 61

FUTURE RESEARCH RECOMMENDATIONS ........................................ 62

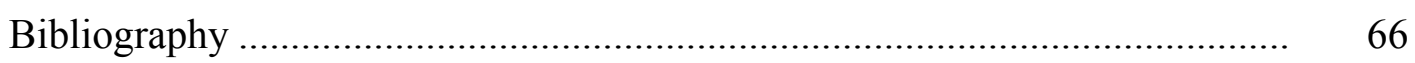

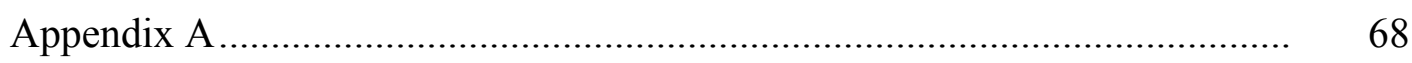

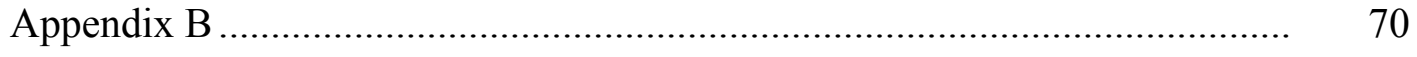

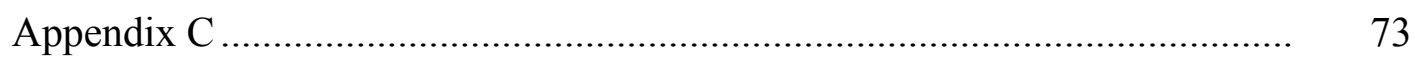

Appendix D ...................................................................................... 85 


\section{LIST OF FIGURES}

Page

Figure 3-1 Diagram of test apparatus for transverse load testing of stoppings in accordance with ASTM E 72 specifications.

Figure 3-2 A 4 × $8 \mathrm{ft}$ section of wall being placed into reaction frame for ASTM E 72 testing

Figure 3-3a A 4 × $8 \mathrm{ft}$ section of wall positioned in the reaction frame for ASTM E 72 transverse load testing

Figure 3-3b Close up view of sections of pipe used as reaction roller to avoid rotational restraint as the wall deflects from the application of transverse load.

Figure 3-4 The wall is not restrained vertically in this free-standing test condition. There is no restraint to the vertical extension of the tension side of the wall 10

Figure 3-5 Apparatus for testing sealant strength on stopping block........... 12

Figure 3-6 Example of sealant test data ................................................. 12

Figure 3-7 Static analysis of two-block sealant strength evaluation............. 14

Figure 3-8 Average computed tensile strength of sealants computed from two-block transverse loading study

Figure 3-9 Sealant seeped into the block joint increasing the apparent thickness of the sealant......

Figure 3-10 Computed transverse load for a 4 x $8 \mathrm{ft}$ section of wall based on extrapolations of the two-block laboratory study....

Figure 3-11 Failure of sealant on stopping wall during full-scale ASTM E 72 test

Figure 4-1 Illustration of rigid-arching of a wall structure 22

Figure 4-2 Analysis for rigid-arching of a wall structure

Figure 4-3 Examination of the rigid-arching loading conditions and expressing the transverse loading as a function of the block compressive strength 
Figure 4-4 Graphical illustration of rigid-arching design equation with an example consisting of a 6-in-thick wall, 72 inches in height, with a material compressive strength of $1,000 \mathrm{psi}$

Figure 4-5. Impact of block orientation and wall height on transverse loading capacity for conventional concrete block used in stopping construction $(1,330 \mathrm{psi}$ compressive strength with $6 \times 8 \times 16$ in nominal dimensions) ......................................... 28

Figure 5-1 NIOSH Mine Roof Simulator load frame ……………………... 30

Figure 5-2 Full-scale mine ventilation stopping wall being tested in the NIOSH Mine Roof Simulator.

Figure 5-3 Diagram illustrating the simulation of rigid arching on a half-wall section of a stopping by biaxial testing in the NIOSH Mine Roof Simulator.

Figure 5-4 Apparatus used to conduct half-wall rigid-arching tests of stopping walls in the NIOSH Mine Roof Simulator .....

Figure 5-5 Block column rests on rolling platform to allow load cells to measure lateral load.................................................................. 34

Figure 5-6 Load cells used to accurately measure lateral load ..................... 34

Figure 5-7 Illustration of how block dimensional tolerances can cause localized loading on a three-block-wide wall (not to scale)........ $\quad 35$

Figure 5-8a Test of a half-wall made from lightweight block (546-psi compressive strength)

Figure 5-8b Test of a half-wall made from conventional concrete block (1,330-psi compressive strength)

Figure 5-9 Close up view of Kingsway, autoclaved, concrete block shows air pockets in the block structure

Figure 5-10 Compressive strength test data for Kingsway block

Figure 5-11 Compressive strength test data for conventional concrete block 
Figure 5-12 Half-wall rigid-arching tests conducted in the Mine Roof Simulator on the Kingsway lightweight block

Figure 5-13 Half-wall rigid-arching tests conducted in the Mine Roof Simulator on conventional concrete block

Figure 5-14 Comparison of MRS half-wall rigid-arch testing to the theoretical prediction of transverse loading for Kingsway block stoppings

Figure 5-15 Comparison or MRS half-wall rigid-arch testing to the theoretical predictions of transverse loading for Klondike block stoppings

Figure 6-1 Diagram of the pressure chamber in the NIOSH Experimental Coal Mine used for conducting full-scale transverse loading tests

Figure 6-2 Data acquisition system to measure air pressure and wall displacement for the full-scale stopping wall testing in the NIOSH Experimental Coal Mine

Figure 6-3 Comparison of half-wall rigid-arch test in the MRS to the full-scale stopping wall test in the NIOSH Experimental Coal Mine for the Kingsway block

Figure 6-4 Comparison of full-scale mine test of Kingsway block with the MRS half-wall regression trend line and suite of MRS tests ......

Figure 6-5 Photo after the wall was destroyed from the transverse loading. Researcher is standing next to the displacement transducers used to measure the wall deflection

Figure 6-6 Arch height shown to occur between top and bottom layer of block that were grouted (cemented) in place in this particular test 
Figure 6-7 Comparison of half-wall rigid-arch test in the Mine Roof Simulator to the full-scale stopping wall test in the NIOSH Experimental Coal Mine for the Klondike block stopping .....

Figure 6-8 Comparison of full-scale mine test of Klondike conventional block with the MRS half-wall regression trend line and suite of MRS tests

Figure 6-9 Photo showing conventional (Klondike) wall after full-scale test in the Experimental Coal Mine noting the arch length between the top and bottom course of block.

Figure 6-10 Photo showing bottom course of the conventional block wall after the test illustrating the crush zone and shearing action developed on the bottom course of block.

Figure 6-11 Close up view of the base of the conventional (Klondike) block wall showing the rotation of the wall and formation of the bottom hinge of the arch

Figure 6-12 Diagram of the longwall gallery at the Lake Lynn limestone mine showing location of stopping constructions in the \#4 and \#5 crosscut......

Figure 6-13a Transverse load testing of a hollow-core stopping at the Lake Lynn Laboratory

Figure 6-13b Transverse load testing of a solid block stopping at the Lake Lynn Laboratory

Figure 6-14 Comparison of Lake Lynn full-scale stopping tests with the MRS rigid-arching tests.

Figure A-1. Transverse load capacity due to weight of the block 68

Figure B-1 Diagram of the Mine Roof Simulator. 70 


\section{LIST OF TABLES}

Page

Table 3-1 Applied load data from two-block sealant testing ........................ 13

Table 3-2 Calculated tensile strength of sealants from two-block study ........ 15

Table 5-1 Specifications for MRS Biaxial Testing on Kingsway block

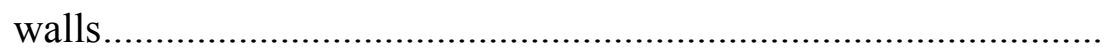

Table 5-2 Specifications for MRS Biaxial Testing on Klondike block

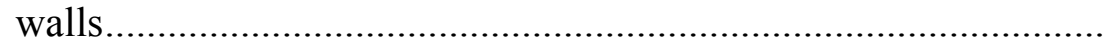




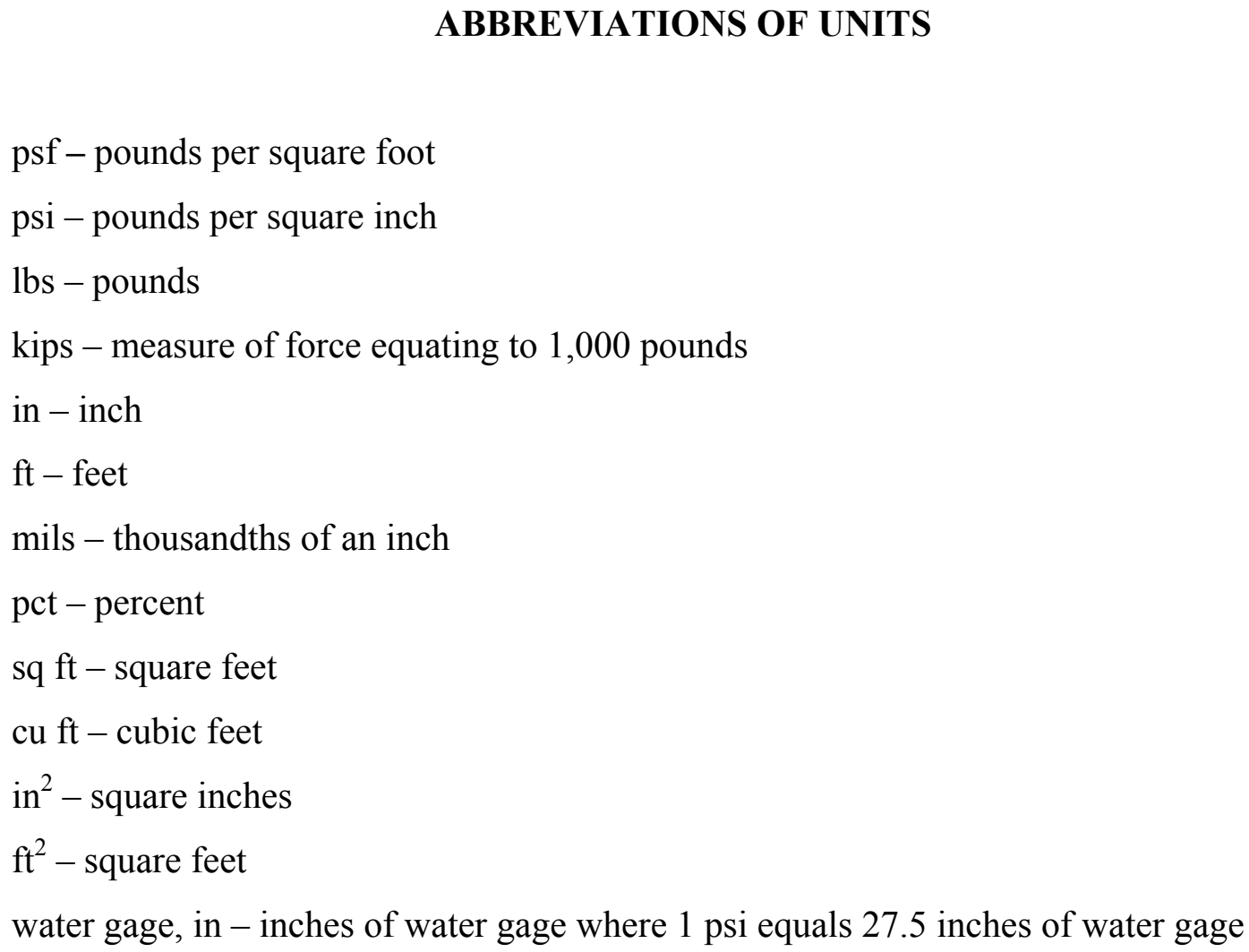




\section{CHAPTER 1: INTRODUCTION}

An effective ventilation system requires a ventilation plan that is not only sound in design, but also well implemented during both mine development and subsequent production stages. Ventilation stoppings control critical ventilation throughout an underground mine and are an integral part of the ventilation system. Operating longwalls in the United States (U.S.) alone require an estimated 21,600 new stoppings each year, and mines using room-and-pillar mining methods will require another 66,000 stoppings (Tien, 1996). With an average cost of $\$ 600$ - $\$ 800$ per stopping, total costs could easily exceed $\$ 90$ million per year for the coal industry (Tien, 1996).

Stoppings are designed primarily to withstand air pressure differentials generated by the mine fan that exert transverse loading against the high-pressure side or face of the stopping. These pressures, typically measured in inches of water gage, are generally less than 7 inches of water in the working sections of the mine, equating to approximately $0.25 \mathrm{psi}$. The pressure increases as the proximity to the mine fan increases. Near the mouth of a bleeder fan, the pressure can exceed 1 psi, which exerts considerable force against the stopping. Air blasts from roof falls can generate localized areas of higher pressure that can destroy stoppings. Seals, with a minimum transverse load capacity of $20 \mathrm{psi}$, are designed to contain explosions, but stoppings also play a role in maintaining ventilation during an explosion. Australia, for example, requires a 5 psi transverse load capacity for permanent stoppings used in main roadways and near sealed areas (Gillies et al., 2001). This is done, in part, to prevent widespread damage to the ventilation system in the event that an explosion does occur.

Unlike seals, which are required to pass full-scale testing to ensure their transverse load capacity, there are no full-scale tests required for stoppings to determine their load capacity. The current Code of Federal Regulations (CFR) requirement is to test $4 \times 8$ - $\mathrm{ft}$ sections of freestanding walls (CFR Part 75.333 Ventilation Controls, 1996). This test inadequately determines the transverse load capacity of stopping constructions since in the mine the walls are restrained by the mine roof and floor, and pillar ribs. This restraint allows for significantly greater transverse loading capability by taking advantage of the 
compressive forces that are generated as the wall arches between the mine roof and floor. As a result, the true transverse load capacities of mine ventilation stoppings are not known.

Recently, a new generation of lightweight blocks has been developed for mine ventilation stopping constructions. While stoppings utilizing these blocks have all passed the current CFR criteria, it is believed that their true transverse load capacity varies considerably. This is because the material strength of the block types vary by as much of an order of magnitude, and the material strength of the blocks correlates to the arching capability of the restrained wall in the mine during transverse loading. Without such knowledge, the design of mine ventilation systems using these lighter-weight, but lowercapacity alternative constructions can be misleading, potentially exposing the mine to inadequate ventilation control under some circumstances.

The objective of this thesis is to develop a new protocol to determine the true transverse load capacity of block stopping constructions. Using the unique biaxial loading capabilities of the National Institute for Occupational Safety and Health's (NIOSH) Mine Roof Simulator (MRS), rigid-arching conditions for stoppings are simulated in the laboratory, using a single column of block to predict the transverse load capacity of an entire stopping. Verification of the procedure has been done through fullscale testing of stoppings in a pressure chamber in the NIOSH Experimental Coal Mine and in the underground Longwall Gallery at the NIOSH Lake Lynn facility. The MRS laboratory testing will allow a full research program to be undertaken to evaluate the proper design parameters for determining the transverse load capacity of stoppings. Ultimately, this will lead to more appropriate design criteria and allow regulatory statutes to be developed that will ensure their safe application for specific conditions in underground mines. 


\section{CHAPTER 2: REVIEW OF LITERATURE}

Masonry structures have been utilized dating back to building of pyramids and other structures 10,000 years ago. Modern masonry type structures using cementitious materials date back over a 100 years. As a result of this long history, considerable research has been conducted on masonry structures. Masonry as a general term involves clay brick or concrete block structures where the unit blocks are mortared together at the joints to from a more continuous structure. Most mine ventilation stoppings are walls constructed from concrete blocks and therefore fit this classification, except that stoppings in recent times are dry-stacked as opposed to having mortared joints. In this regard, the research to support this thesis is considered an extension of what has previously been done in this general area.

A comprehensive assessment of masonry design is found in a book authored by Drysdale, et al. (1994) entitled "Masonry Structures: Behavior and Design". The flexural behavior of masonry walls is thoroughly addressed in chapter 7. Transverse loading due to wind pressure is analyzed for walls spanning vertically between lateral supports along the top and bottom edges of the wall face. In two dimensions, this condition equates to a simply supported beam. The flexural strength of such walls is determined by the tensile strength of the mortared joints, generally at the mid span of the wall. The current U.S. CFR requirements for transverse loading of stoppings (CFR Part 75.333 Ventilation Controls, 1996) are an extension of this flexural analysis, except the CFR assumes drystacked block construction for mine ventilation stoppings, which therefore have no tensile strength. The CFR requires testing of freestanding walls with sealant applied to the lowpressure face of the wall to provide the tensile strength necessary to resist the moment induced by the transverse pressure.

Drysdale also addresses the impact of axial loading on the wall. For a dry-stacked wall, axial loading can significantly increase the transverse load capacity by resisting the bending moment induced by the transverse load. In this sense, the axial load can take the

place of the lack of tensile strength in these dry-stacked configurations. This is why ground pressures acting on a stopping wall will greatly increase their transverse load capacity. Drysdale also describes a unity equation for combined axial and transverse 
loading of walls. The unity equation 2.1 is present in some masonry codes and requires that the combined compressive stresses from axial loading and bending must be limited to the material strength to achieve proper design under these conditions.

$$
\frac{f_{a}}{F_{a}}+\frac{f_{b}}{F_{b}}=1
$$

Where: $f_{\mathrm{a}}, f_{\mathrm{b}}=$ compressive stresses due to applied axial load and bending, respectively, and

$\mathrm{F}_{\mathrm{a}}, \mathrm{F}_{\mathrm{b}}=$ allowable axial and bending compressive stresses, respectively.

The loading mechanism being addressed in this thesis involves arching of the wall structure. Drysdale also devotes a section of the book to the arching of walls. Generally, arching can be described as bridging between two rigid abutments as opposed to unrestrained end conditions. Studies into the arching behavior of masonry date back to 1951. The Armour Research Foundation, in conjunction with work sponsored by the U.S. Air Force and technically monitored by the Special Studies Office of the Installation Division, Air Material Command, first reported on an investigation of the arching theory. At this time, arching was a radical departure from conventional beam deflection theory that was typically used to evaluate the resistance of masonry walls to wind-generated or some other form of lateral loading.

McDowell has reported on this work in a paper published in the Proceedings of the American Society for Civil Engineers (McDowell et al., 1956). This was the first comprehensive paper published in a trade journal on this subject. McDowell showed that arching can be used to explain the significantly higher lateral loads that brick beams are capable of withstanding than conventional bending analysis would allow. In conventional bending analysis, beams strengths relative to lateral loading are controlled by the tensile properties of the material. This works well for steel beams since steel has a high tensile strength. Conversely, the tensile strength of concrete is generally about one tenth of its compressive strength, so masonry structures cannot depend solely on the tensile strength of the construction material to resist bending or provide for large lateral loading of wall structures. 
McDowell proposed that a three-hinge arch is formed and that the resistance of the wall to lateral loading is due entirely to the tendency of the masonry to crush at the mid span and end supports due to the arching action. The masonry material is assumed to be unable to withstand tensile stress. McDowell describes the transverse loading of a wall as follows. Immediately upon loading, cracks develop on the tension side at the ends and center of the span. Initially, these cracks extend to the centerline of the beam (wall). During subsequent motion, it is assumed that each half of the wall remains rigid and rotates about an end and where the two half walls meet at the center of the wall. The resistance to this motion comes about through a force couple set up at the ends and center due to crushing of the masonry at these positions. The rotation continues until the resisting couple vanishes (i.e., the material fails) or the load is removed.

McDowell also reported on a series of tests conducted at the Massachusetts Institute of Technology where 17 brick beams were tested under fixed-end conditions (Massachusetts Institute of Technology, 1954). These tests were consistent with the arching theory. The ultimate lateral strength of the beams was shown to correlate to the compressive strength of the material. The transverse load capacity was six times greater than what a simply supported beam analysis predicts.

Anderson (1984) examined the theory of arching in more detail by comparing the behavior of masonry walls during the initial loading prior to cracking of the wall and post-cracking behavior of the wall. He concluded that the load required to cause cracking of a wall with rigid abutments can be three times greater than a wall without arching restraint. He also concluded that the ultimate (post-cracking) transverse load capacity of a wall with abutments was three to nine times more than the pre-cracking load. Anderson showed the significance of the stiffness of the abutments in a theoretical analysis of arching and concluded that reducing the stiffness of the abutments will allow greater wall deflections to occur; and a theoretical limit of stability exists where the deflection is too large to generate an arching thrust. Anderson developed an equation relating the arching thrust to the transverse load. This relationship is used in this thesis with modified coefficients to account for the physical characteristics of mine ventilation stoppings.

$$
q_{\text {lat }}=\frac{f_{k}}{\gamma_{m}} \times\left(\frac{t}{L}\right)^{2}
$$




$$
\text { Where } \begin{aligned}
\mathrm{q}_{\mathrm{lat}} & =\text { design lateral strength per unit area of wall, psi, } \\
f_{\mathrm{k}} & =\text { characteristic compressive strength of the masonry, psi, } \\
\gamma_{\mathrm{m}} & =\text { material safety factor, } \\
\mathrm{t} & =\text { wall thickness, in, and } \\
\mathrm{L} & =\text { span of the wall, in. }
\end{aligned}
$$

Through these and related efforts, arching has been recognized as a valid loading mechanism and design consideration for walls bridging rigid abutments. The British Codes of Practice (British Standards Institution, 1978) first recognized arching as a design mechanism in 1978. Curiously, arching is not recognized in the U.S. Masonry Designers Guide (Masonry Designer's Guide: Based on Building Code Requirements for Masonry Structures (ACI 530-92/ASCE, 5-92/TMS 402-92) and Specifications for Masonry Structures (ACI 530.1-92/ASCE 6-93/TMS 602-92)). The design formula specified for arching in the British Codes of Practice is of the same form as that developed by Anderson (equation 2.2). Close contact between the wall and the end abutments must be maintained for these criteria to be applicable in the British code. For vertical spanning walls, such as a wall spanning between a floor and roof, the design code requires that the dead weight vertical load be sufficient to sustain the arching. This work forms the basis for the rigid-arching assessment of stopping walls pursued in this thesis.

The U.S. Bureau of Mines also conducted research on stopping behavior dating back to the 1960's (Kawenski and Mitchell, 1966). The emphasis of this work was primarily on the leakage of stoppings as a result of structural damage from either transverse loading or by ground movements. Fundamental construction techniques were examined and although full-scale tests of transverse loading were conducted, a study into the loading mechanics was not done during this period.

Recently, the concept of arching has also been applied to seal behavior (Sapko, et al., 2003). Tests conducted on seals in a hydrostatic chamber indicated that arching is occurring across the width of the seal, in this case the restraint provided by the pillar ribs. Initial tests showed good agreement of the ultimate transverse loading pressure of the seal to the arching mechanics described in equation 2.2. Research continues in this area to develop scaling factors for various materials and seal thicknesses. 


\section{CHAPTER 3: CURRENT CFR CRITERIA FOR STOPPINGS}

Part 75.333 Ventilation Controls of the CFR requires that permanent ventilation control structures and mine stoppings for underground coal mines be constructed in a traditionally accepted method. Materials that have been tested and shown to have greater or equal strength than traditionally accepted in-mine control structures must be used. While this is somewhat vague, the statute goes on to specify that alternative stopping technologies be tested in accordance with ASTM E 72-80, "Standard Methods of Conducting Strength Tests of Panels for Building Construction”, Section 12 - Transverse Load - Specimen Vertical (ASTM Designation E 72-80, 1981).

\subsection{ASTM E 72 TEST SPECIFICATIONS}

The procedure requires testing of a nominal 4-ft-wide section of wall of a height equal to the mining height where the stopping will be used. Hence, for an 8 - $\mathrm{ft}$ mining height, a $4 \times 8$-ft section of wall would be tested. The wall is to be constructed in the manner it will be used in the mine, including the application of sealant when specified. The test apparatus is shown in the diagram illustrated in figure 3-1. Figure 3-2 shows a wall section being placed into the reaction frame for ASTM E 72 testing at a commercial laboratory (Professional Services Industries) in Pittsburgh, PA. As seen from these figures, the freestanding wall is tested in a vertical orientation. The wall is placed on a steel channel which rests on a cylindrical roller (figure 3-3a) to prevent restrained end conditions. The axis of the roller is parallel to the face of the wall, allowing rotation to occur without restraint, as the wall is deformed from the application of transverse load. Two reaction rollers and contact plates positioned at the top and bottom of the wall allow the wall to deflect under the application of transverse load from the opposite face (figure 3-3b). Again, rollers are utilized to prevent longitudinal restraint as the wall deflects. Transverse loading is applied across the width of the wall through a steel contact plate at quarter-height points of the wall. Rollers in the form of a steel pipe are again used to transfer load from a central I-beam through the contact plates, again to prevent any rotational restraint from occurring. As the load is applied, it is required that the load be recorded as a function of the displacement at the mid-span of the wall height. 
The maximum load normalized to the square foot area of the wall is then defined as the transverse load capacity for the wall. It is also required that three separate walls be tested. The average transverse load capacity from these three tests must exceed 39 psf to comply with the CFR statute.
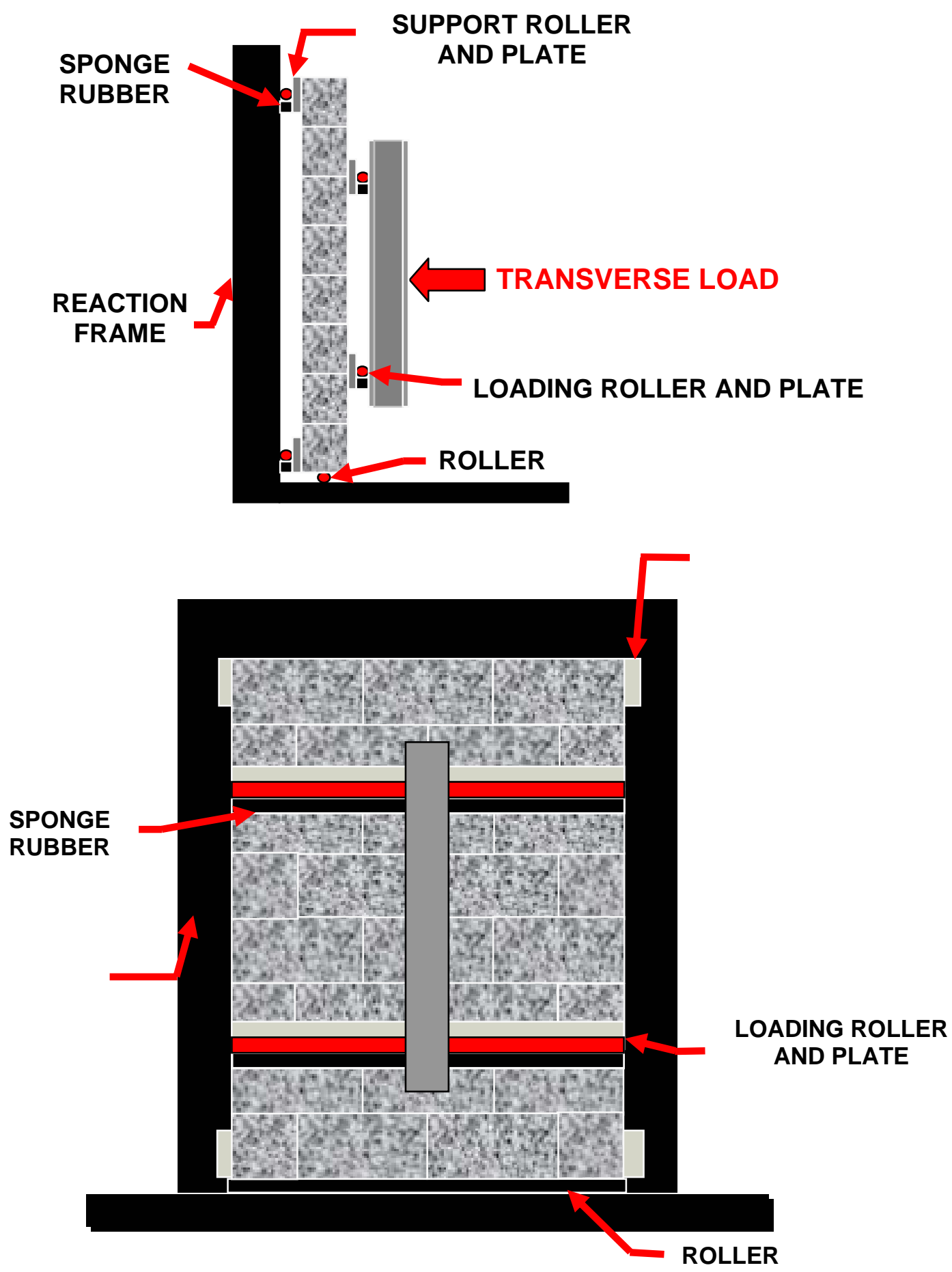

Figure 3-1. Diagram of test apparatus for transverse load testing of stoppings in accordance with ASTM - E 72 specifications. 


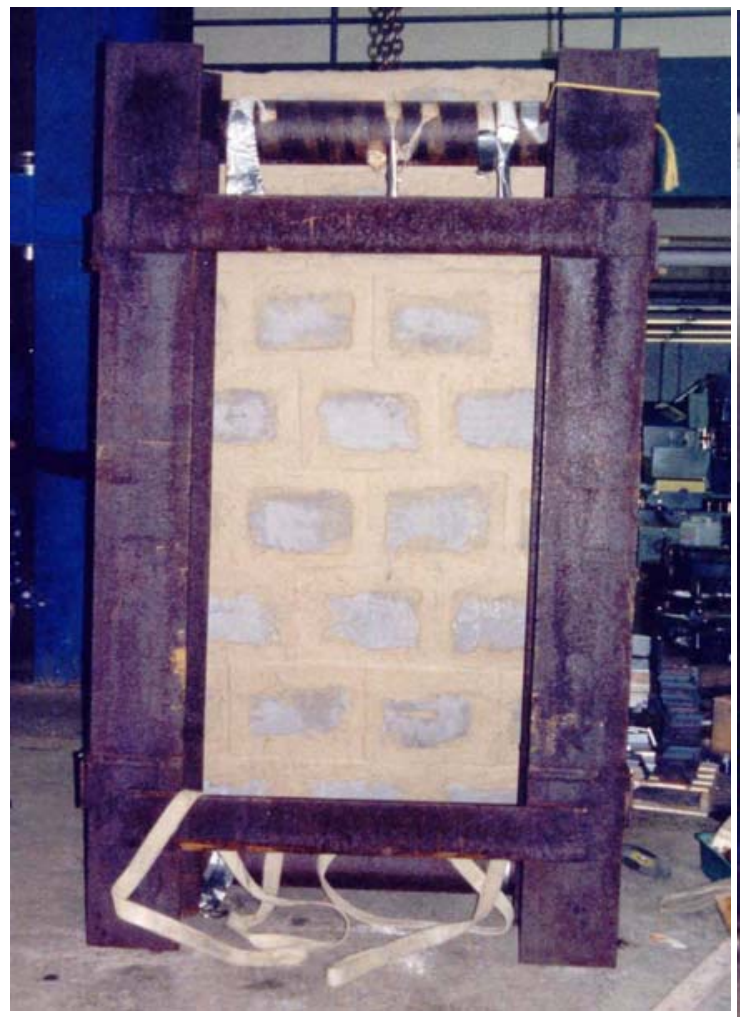

Figure 3-2. A $4 \times 8 \mathrm{ft}$ section of wall being placed into reaction frame for ASTM E 72 testing.

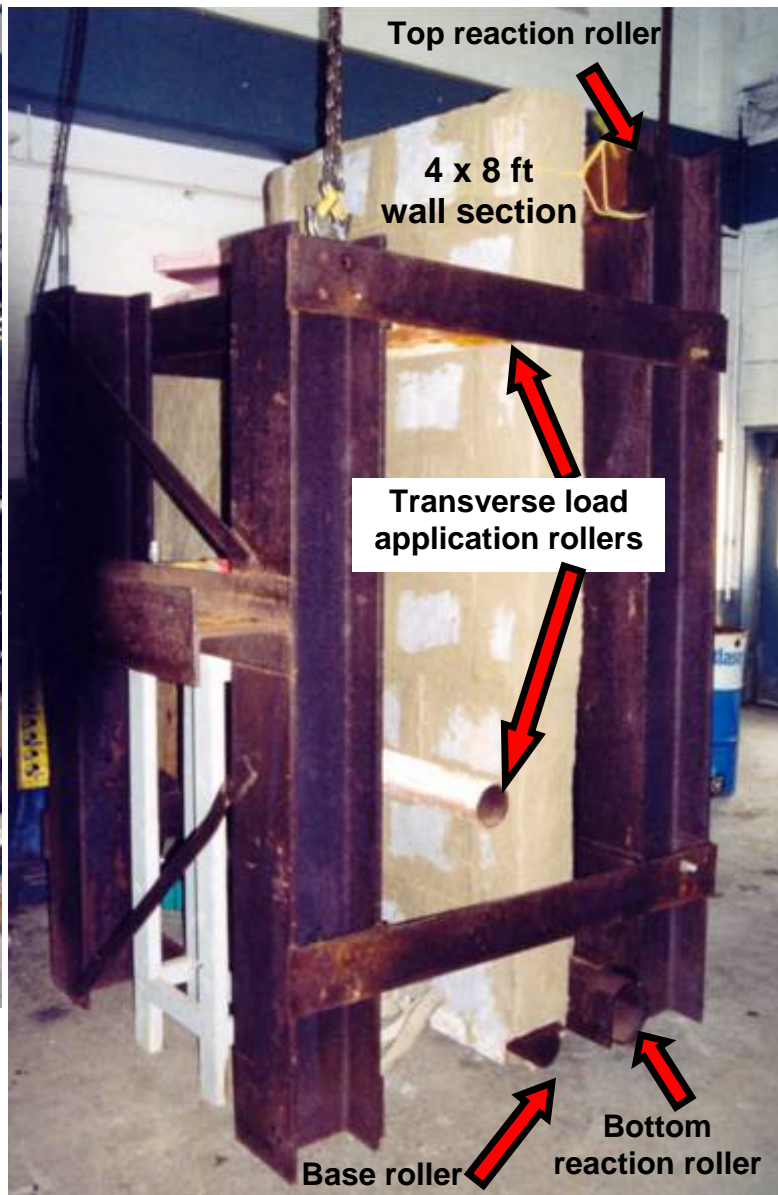

Figure 3-3a. A 4 × $8 \mathrm{ft}$ section of wall positioned in the reaction frame for ASTM E 72 transverse load testing.

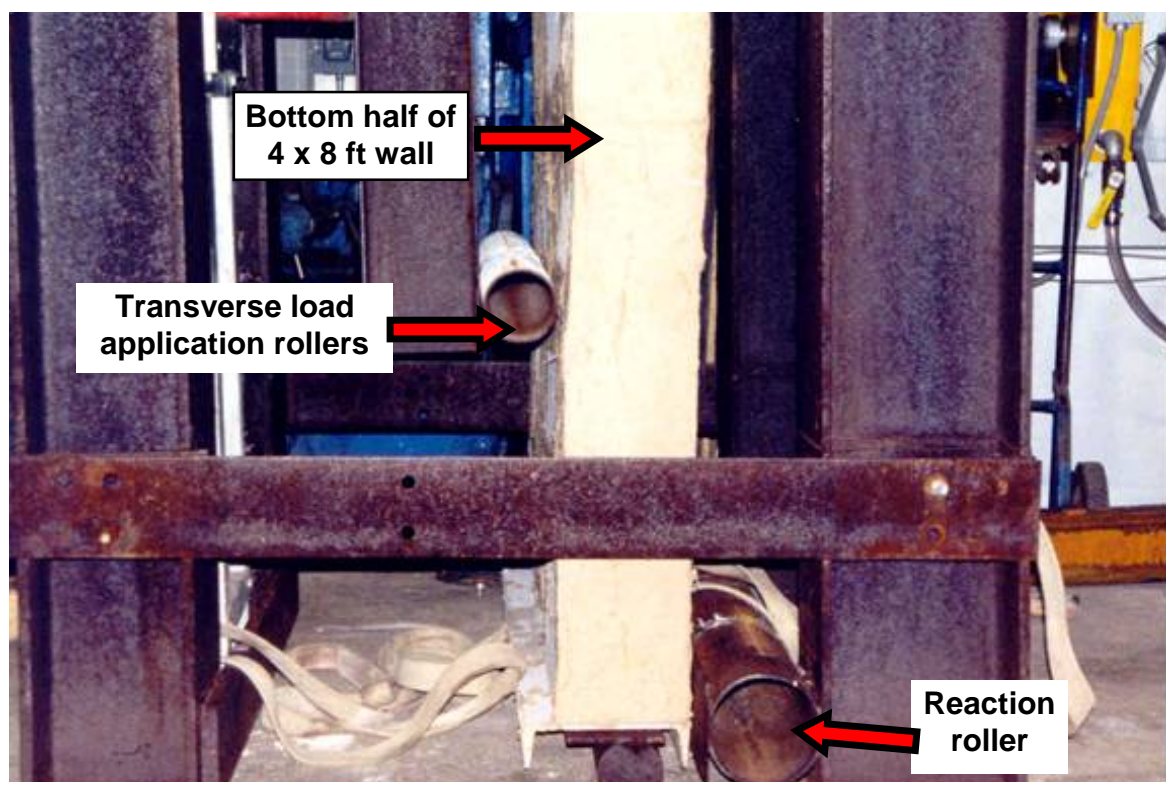

Figure 3-3b. Close up view of sections of pipe used as reaction roller to avoid rotational restraint as the wall deflects from the application of transverse load. 


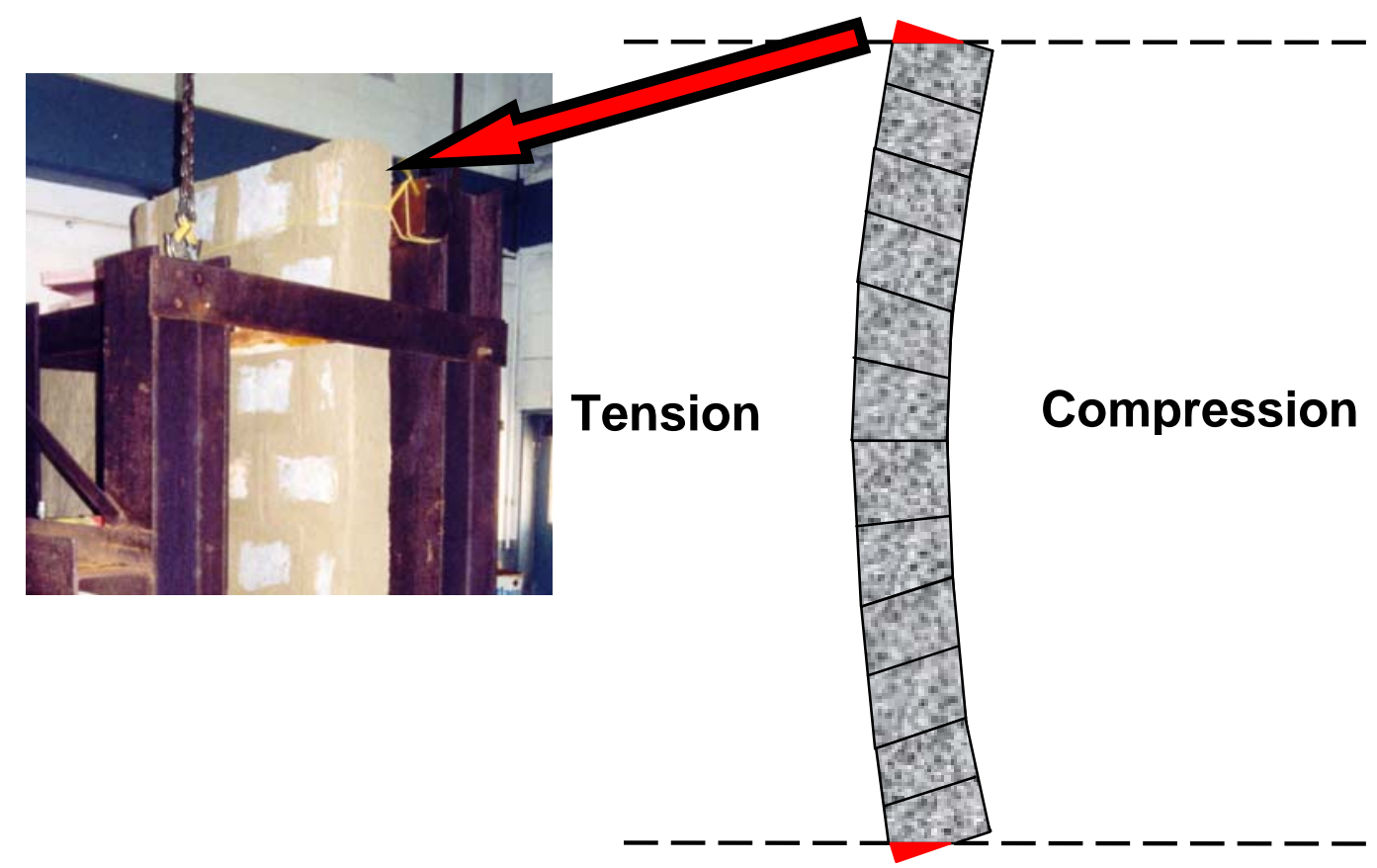

Figure 3-4. The wall is not restrained vertically in this free-standing test condition. There is no restraint to the vertical extension of the tension side of the wall.

\subsection{INADEQUACIES OF CURRENT CFR SPECIFICATIONS}

Examination of the mechanics of the wall response to transverse loading reveals the inadequacies of the CFR test procedure. First, it is seen that great care is taken to ensure that there is no longitudinal restraint provided to the wall as the load is applied. Essentially, the wall is considered freestanding and unrestrained from vertical movement as it bends from the application of transverse load (figure 3-4). The objective of the test is to evaluate the flexural strength of the wall. Any structure that is subject to bending produces tensile stresses on one side of the structure and compressive stresses on the opposite side of the structure (figure 3-4). Typically, the tensile strength of the material, being weaker than the compressive strength, controls the capability of the structure to withstand loads that produce bending. Concrete has relatively little tensile strength, but a dry-stacked block stopping has no effective tensile strength since the joints are not bonded. Theoretically, the transverse load capacity of a freestanding, dry-stacked stopping would be provided only from the weight of the block, which acts to provide a superimposed vertical load on the structure. Even the heaviest blocks would not provide enough axial loading to meet the 39-psf criteria in the CFR. Appendix A documents the 
theoretical assessment of the contribution of the block weight to the transverse load capacity of a dry-stacked stopping.

The tensile strength is actually provided by the application of sealant to the face of the wall. This brings up a few more points of discussion. First, this is obviously not the primary function of the sealant. As such, there was little information available about the tensile strengths of sealants prior to this research, and in fact, evaluating or knowing the strength of the sealant is not part of CFR test requirement. Since the sealant under these conditions is providing the major contribution to the transverse load capacity of the drystacked block stopping, the placement of the sealant is also critical to the test results. In order for the sealant to be effective in controlling the transverse loading, it must be applied to the face opposite the load application, i.e., the low-pressure side of the stopping in the mine environment. If the ventilation could be reversed either intentionally or unintentionally, then the sealant should be applied to both sides of the stopping under these criteria. Since several sealants are available each with different material properties, then the stopping should only be certified with a specific sealant as used in the test. Furthermore, for a given sealant, the thickness of the sealant contributes significantly to the effective tensile strength and resulting transverse load capacity of the wall. How thick the sealant is applied in the test program compared to the thickness normally applied to such stoppings in the mine is another issue of concern. The test program should exclude abnormally thick sealant applications.

\subsection{EVALUATION OF SEALANT TENSILE STRENGTH CHARACTERISTICS}

Since the sealant plays a major role in the transverse load capacity of a freestanding wall, the tensile strength of several commonly used sealants were determined as part of this study. The apparatus for conducting these tests is shown in figure 3-5. A sixinch-wide section of sealant was placed across the joint of two full-size, hollow-core concrete blocks. A small hydraulic ram was used to apply loading at the joint on the face opposite the sealant. A displacement transducer was used to measure the deflection at the joint. The control parameters in the test were the thickness of the sealant and the curing time. Two thicknesses were evaluated: $1 / 8$ and $1 / 4$ in. Three curing times were evaluated: 7 days, 14 days, and 28 days. Figure 3-6 shows an example of the test data. 
The resulting data for eight different sealants are shown in table 3-1. Using this data, the tensile strength of the sealant was computed using the static analysis illustrated in figure 3-7. The force $(\mathrm{T})$ acting across the sealant joint is calculated from the applied load $\left(\mathrm{F}_{\mathrm{r}}\right)$ and the block dimensions by summing moments. The tensile strength is computed by dividing the force $(\mathrm{T})$ by the area of the sealant along the joint (i.e., sealant thickness times the block length). These results are documented in table 3-2. The applied loading and average computed tensile strength for each sealant is shown in the chart in figure 3-8.
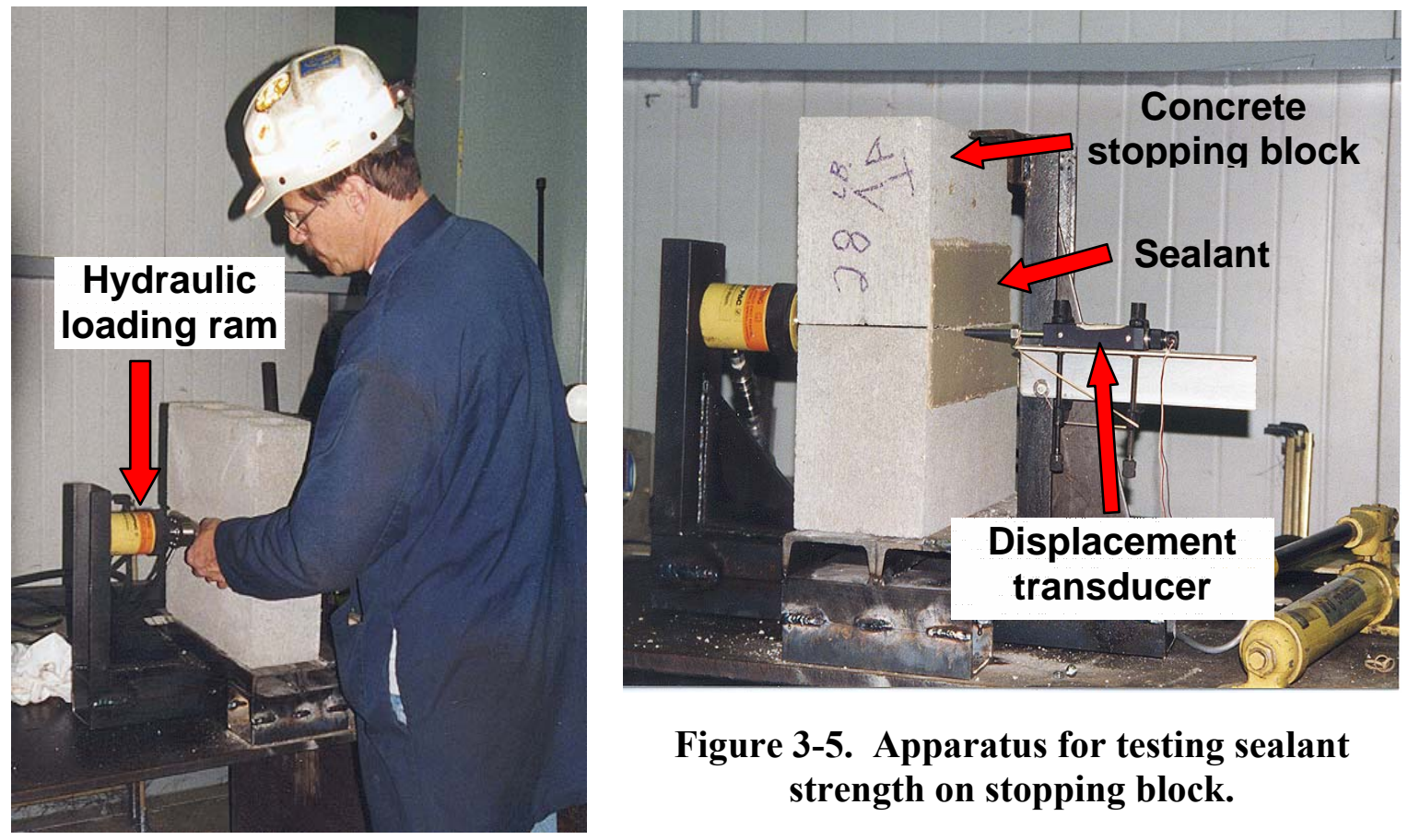

Figure 3-5. Apparatus for testing sealant strength on stopping block.

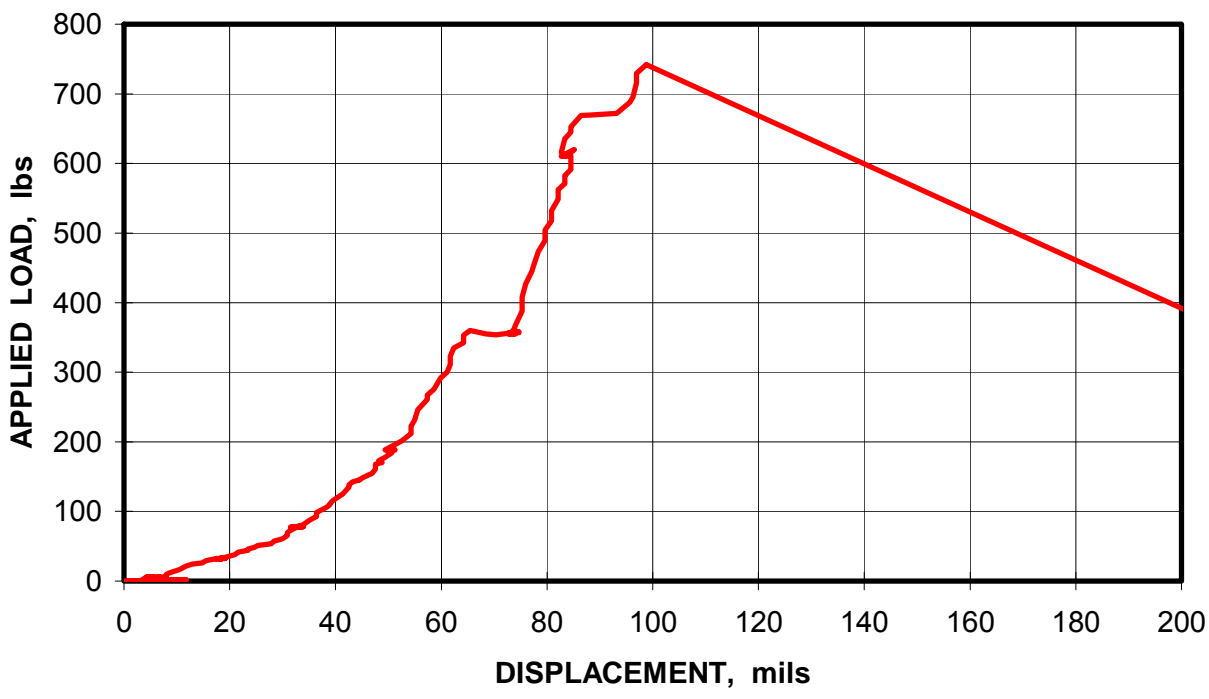

Figure 3-6. Example of sealant test data. 
Table 3-1. Applied load data from two-block sealant testing.

\begin{tabular}{|c|c|c|c|c|c|c|}
\hline \multirow{2}{*}{ Sealant Type } & \multirow{2}{*}{$\begin{array}{c}\text { Sealant Thickness - } \\
\text { Cure Time }\end{array}$} & \multicolumn{5}{|c|}{ Applied Load, lbs } \\
\hline & & Test \#1 & Test \#2 & Average & Standard Deviation & Maximum \\
\hline DUPONT YTF & $1 / 8$ in -7 days & 808.30 & 533.58 & 670.94 & 194.26 & 808.30 \\
\hline DUPONT YTF & $1 / 8$ in -14 days & 741.76 & 357.14 & 549.45 & 271.96 & 741.76 \\
\hline DUPONT YTF & $1 / 8$ in -28 days & 948.72 & 934.68 & 941.70 & 9.93 & 948.72 \\
\hline DUPONT YTF & $1 / 4$ in -7 days & 459.10 & 421.86 & 440.48 & 26.33 & 459.10 \\
\hline DUPONT YTF & $1 / 4$ in -14 days & 422.47 & 719.17 & 570.82 & 209.80 & 422.47 \\
\hline DUPONT YTF & $1 / 4$ in - 28 days & 968.86 & 766.79 & 867.83 & 142.89 & 968.86 \\
\hline DUPONT YTF & Averages & & & 673.53 & 142.53 & 724.87 \\
\hline OMEGA & $1 / 8$ in -7 davs & 212.45 & 286.32 & 249.39 & 52.23 & 286.32 \\
\hline OMEGA & $1 / 8$ in -14 days & 175.21 & 385.23 & 280.22 & 148.50 & 385.23 \\
\hline OMEGA & $1 / 8$ in -28 days & 463.37 & 548.23 & 505.80 & 60.00 & 548.23 \\
\hline OMEGA & $1 / 4$ in -7 days & 326.01 & 406.59 & 366.30 & 56.98 & 406.59 \\
\hline OMEGA & $1 / 4$ in -14 days & 494.51 & 415.14 & 454.82 & 56.12 & 494.51 \\
\hline OMEGA & $1 / 4$ in -28 days & 542.12 & 589.13 & 565.63 & 33.24 & 589.13 \\
\hline OMEGA & Averages & & & 403.69 & 67.85 & 451.67 \\
\hline EAGLE O & $1 / 8$ in -7 days & 410.87 & 558.61 & 484.74 & 104.47 & 558.61 \\
\hline EAGLE O & $1 / 8$ in -14 days & 532.97 & 693.53 & 613.25 & 113.53 & 693.53 \\
\hline EAGLE O & $1 / 8$ in -28 days & 106.84 & 311.97 & 209.40 & 145.05 & 311.97 \\
\hline EAGLE O & $1 / 4$ in -7 days & 441.39 & 337.61 & 389.50 & 73.39 & 441.39 \\
\hline EAGLE O & $1 / 4$ in -14 days & 683.15 & 342.49 & 512.82 & 240.88 & 683.15 \\
\hline EAGLE O & $1 / 4$ in -28 days & 457.27 & 158.12 & 307.69 & 211.53 & 457.27 \\
\hline EAGLE O & Averages & & & 419.57 & 148.14 & 524.32 \\
\hline PYRO TNT & $1 / 8$ in -7 davs & 630.65 & 101.95 & 366.30 & 373.84 & 630.65 \\
\hline PYRO TNT & $1 / 8$ in -14 days & 53.42 & 148.35 & 100.89 & 67.13 & 148.35 \\
\hline PYRO TNT & $1 / 8$ in -28 days & 97.07 & 145.91 & 121.49 & 34.54 & 145.91 \\
\hline PYRO TNT & $1 / 4$ in -7 days & 524.42 & 423.08 & 473.75 & 71.66 & 524.42 \\
\hline PYRO TNT & $1 / 4$ in -14 days & 564.10 & 308.91 & 436.51 & 180.45 & 564.10 \\
\hline PYRO TNT & $1 / 4$ in - 28 days & 321.73 & 124.54 & 223.14 & 139.44 & 321.73 \\
\hline PYRO TNT & Averages & & & 287.01 & 144.51 & 389.19 \\
\hline PYRO SNT & $1 / 8$ in -7 days & 469.48 & 781.44 & 625.46 & 220.59 & 781.44 \\
\hline PYRO SNT & $1 / 8$ in -14 days & 425.82 & 239.93 & 332.88 & 131.45 & 425.82 \\
\hline PYRO SNT & $1 / 8$ in -28 days & 108.67 & 1199.02 & 653.85 & 771.00 & 1199.02 \\
\hline PYRO SNT & $1 / 4$ in -7 days & 580.59 & 402.01 & 491.30 & 126.27 & 580.59 \\
\hline PYRO SNT & $1 / 4$ in -14 days & 973.44 & 529.61 & 751.53 & 313.84 & 973.44 \\
\hline PYRO SNT & $1 / 4$ in - 28 days & 1257.02 & 344.32 & 800.67 & 645.38 & 1257.02 \\
\hline PYRO SNT & Averages & & & 609.28 & 368.09 & 869.56 \\
\hline EAGLE AIR & $1 / 8$ in -7 days & 788.77 & 939.56 & 864.16 & 106.63 & 939.56 \\
\hline EAGLE AIR & $1 / 8$ in -14 days & 700.85 & 1115.69 & 908.27 & 293.33 & 1115.69 \\
\hline EAGLE AIR & $1 / 8$ in -28 days & & 987.18 & 987.18 & & 987.18 \\
\hline EAGLE AIR & $1 / 4$ in -7 days & 706.04 & 849.51 & 777.78 & 101.45 & 849.51 \\
\hline EAGLE AIR & $1 / 4$ in -14 days & 545.79 & 867.52 & 706.65 & 227.50 & 867.52 \\
\hline EAGLE AIR & $1 / 4$ in -28 days & 949.94 & 491.45 & 720.70 & 324.20 & 949.94 \\
\hline EAGLE AIR & Averages & & & 812.94 & 210.62 & 951.57 \\
\hline RITE-CRETE & $1 / 8$ in -7 days & 36.63 & 60.74 & 48.69 & 17.05 & 60.74 \\
\hline RITE-CRETE & $1 / 8$ in -14 days & 54.3346 & & 54.33 & & 54.33 \\
\hline RITE-CRETE & $1 / 8$ in -28 days & 40.90 & 28.08 & 34.49 & 9.07 & 40.90 \\
\hline RITE-CRETE & $1 / 4$ in -7 days & 72.34 & 89.74 & 81.04 & 12.30 & 89.74 \\
\hline RITE-CRETE & $1 / 4$ in -14 days & No Sam & es -- Broke & moving & & \\
\hline RITE-CRETE & $1 / 4$ in -28 days & 68.38 & 56.17 & 62.27 & 8.63 & 68.38 \\
\hline RITE-CRETE & Averages & & & 56.37 & 11.76 & 62.82 \\
\hline B-BOND & $1 / 8$ in -7 days & 358.97 & BROKEN & 358.97 & & 358.97 \\
\hline B-BOND & $1 / 8$ in -14 days & 244.81 & 157.51 & 201.16 & 61.73 & 244.81 \\
\hline B-BOND & $1 / 8$ in -28 days & 126.98 & 121.18 & 124.08 & 4.10 & 126.98 \\
\hline B-BOND & $1 / 4$ in -7 days & 333.03 & 339.44 & 336.23 & 4.53 & 339.44 \\
\hline B-BOND & $1 / 4$ in -14 days & 538.46 & 429.79 & 484.13 & 76.84 & 538.46 \\
\hline B-BOND & $1 / 4$ in - 28 days & 199.63 & 543.35 & 371.49 & 243.04 & 543.35 \\
\hline B-BOND & Averages & & & 308.47 & 78.05 & 358.67 \\
\hline
\end{tabular}




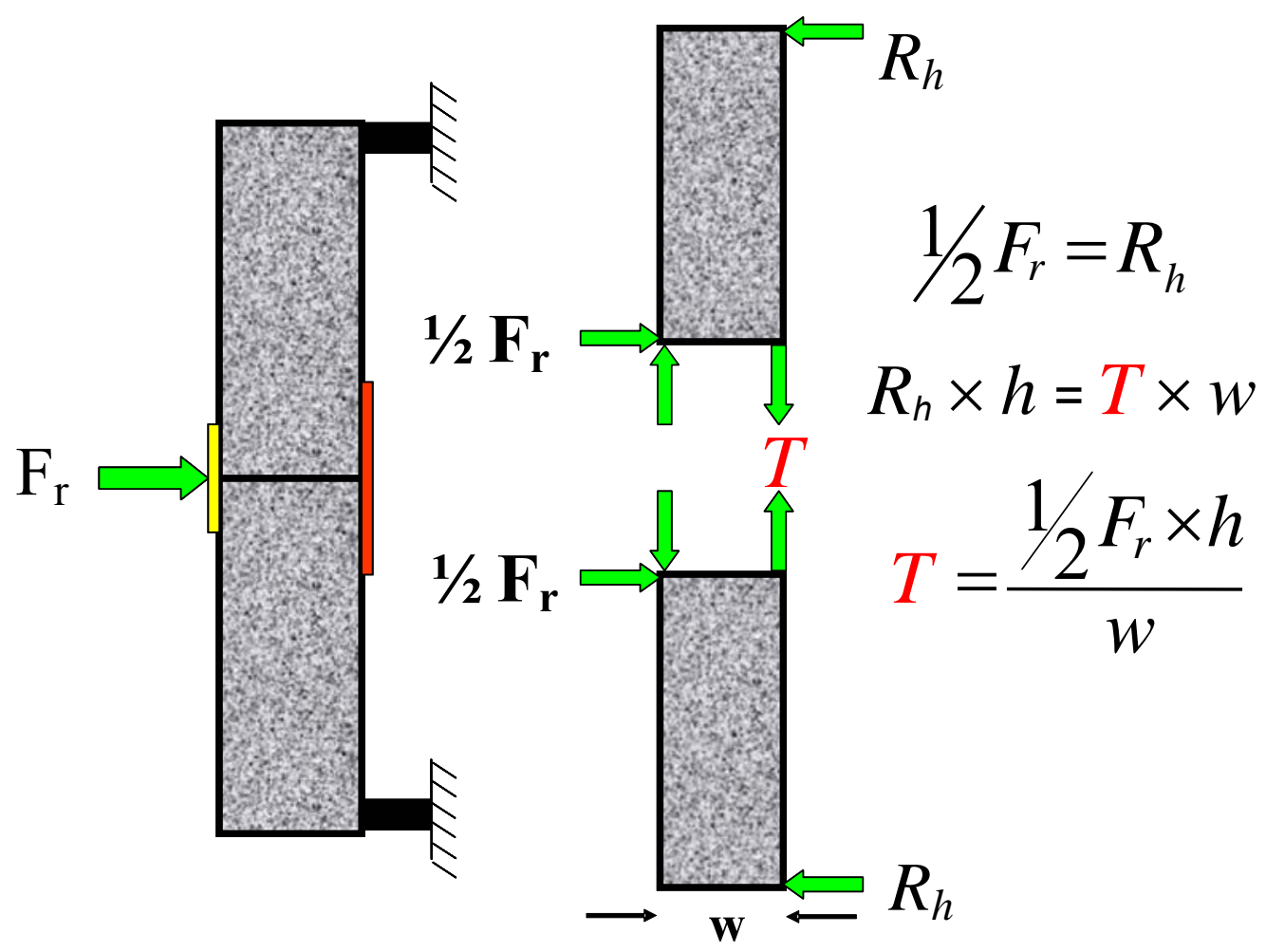

Figure 3-7. Static analysis of two-block sealant strength evaluation.

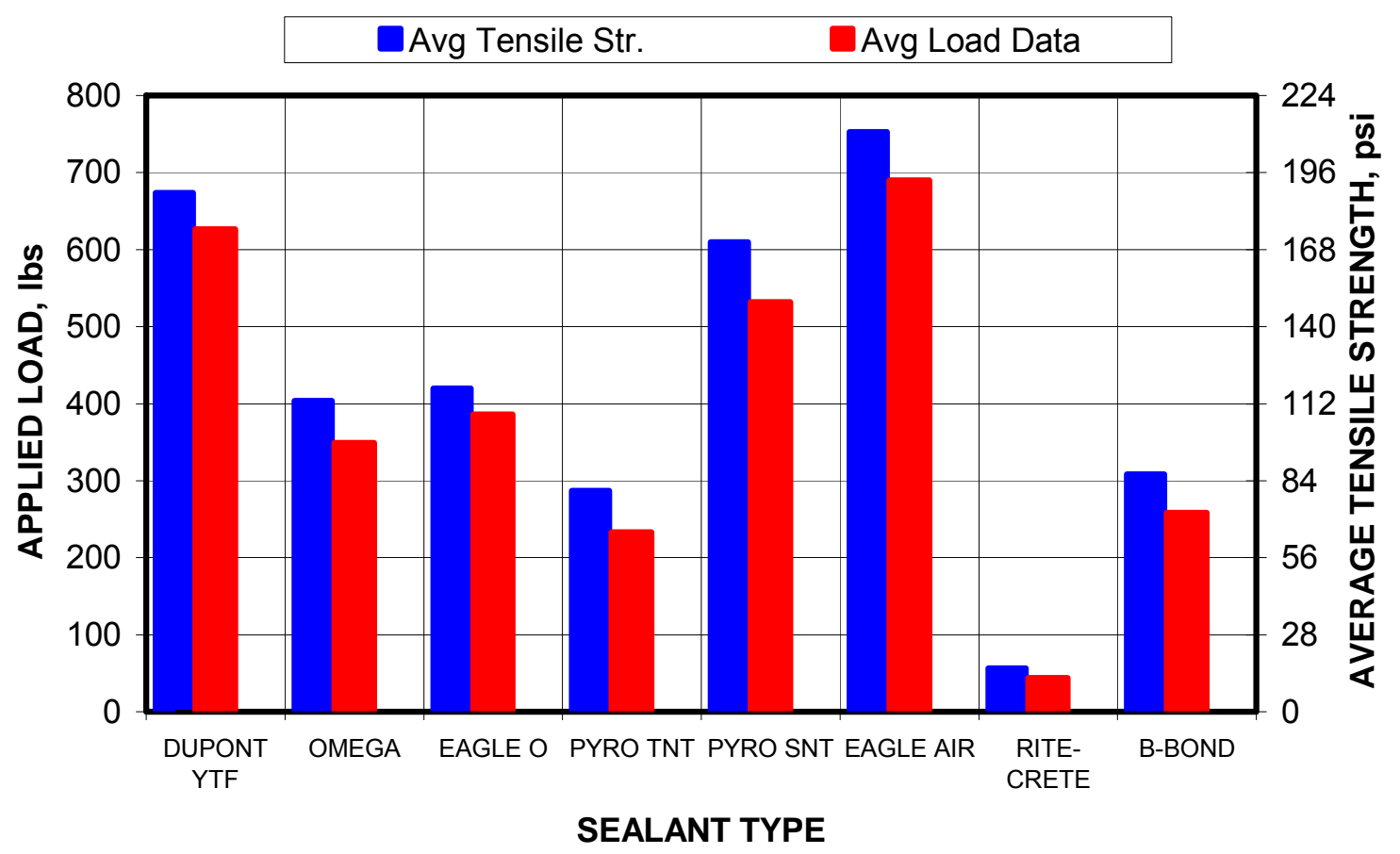

Figure 3-8. Average computed tensile strength of sealants computed from two-block transverse loading study. 
Table 3-2. Calculated tensile strength of sealants from two-block study.

\begin{tabular}{|c|c|c|c|c|c|c|}
\hline \multirow{2}{*}{ Sealant Type } & \multirow{2}{*}{$\begin{array}{c}\text { Sealant Thickness - } \\
\text { Cure Time }\end{array}$} & \multicolumn{5}{|c|}{ Calculated Tensile Strength, psi } \\
\hline & & Test \#1 & Test \#2 & Average & Standard Deviation & Maximum \\
\hline DUPONT YTF & $1 / 8$ in -7 days & 274.28 & 181.06 & 227.67 & 65.92 & 274.28 \\
\hline DUPONT YTF & $1 / 8$ in -14 days & 251.70 & 121.19 & 186.45 & 92.29 & 251.70 \\
\hline DUPONT YTF & $1 / 8$ in -28 days & 321.93 & 317.17 & 319.55 & 3.37 & 321.93 \\
\hline DUPONT YTF & $1 / 4$ in -7 days & 77.89 & 71.57 & 74.73 & 4.47 & 77.89 \\
\hline DUPONT YTF & $1 / 4$ in -14 days & 71.68 & 122.02 & 96.85 & 35.60 & 122.02 \\
\hline DUPONT YTF & $1 / 4$ in - 28 days & 164.38 & 130.10 & 147.24 & 24.24 & 164.38 \\
\hline DUPONT YTF & Averages & & & 175.42 & 37.65 & 202.04 \\
\hline OMEGA & $1 / 8$ in -7 days & 72.09 & 97.16 & 84.63 & 17.72 & 97.16 \\
\hline OMEGA & $1 / 8$ in -14 days & 59.46 & 130.72 & 95.09 & 50.39 & 130.72 \\
\hline OMEGA & $1 / 8$ in -28 days & 157.24 & 186.03 & 171.63 & 20.36 & 186.03 \\
\hline OMEGA & $1 / 4$ in -7 days & 55.31 & 68.99 & 62.15 & 9.67 & 68.99 \\
\hline OMEGA & $1 / 4$ in -14 days & 83.90 & 70.44 & 77.17 & 9.52 & 83.90 \\
\hline OMEGA & $1 / 4$ in - 28 days & 91.98 & 99.96 & 95.97 & 5.64 & 99.96 \\
\hline OMEGA & Averages & & & 97.77 & 18.88 & 111.13 \\
\hline EAGLE O & $1 / 8$ in -7 days & 139.42 & 189.55 & 164.49 & 35.45 & 189.55 \\
\hline EAGLE O & $1 / 8$ in -14 days & 180.85 & 235.34 & 208.10 & 38.53 & 235.34 \\
\hline EAGLE O & $1 / 8$ in -28 days & 36.25 & 105.86 & 71.06 & 49.22 & 105.86 \\
\hline EAGLE O & $1 / 4$ in - 7 days & 74.89 & 57.28 & 66.09 & 12.45 & 74.89 \\
\hline EAGLE O & $1 / 4$ in -14 days & 115.91 & 58.11 & 87.01 & 40.87 & 115.91 \\
\hline EAGLE O & $1 / 4$ in - 28 days & 77.58 & 26.83 & 52.21 & 35.89 & 77.58 \\
\hline EAGLE O & Averages & & & 108.16 & 35.40 & 133.19 \\
\hline PYRO TNT & $1 / 8$ in -7 days & 214.00 & 34.60 & 124.30 & 126.86 & 214.00 \\
\hline PYRO TNT & $1 / 8$ in -14 days & 18.13 & 50.34 & 34.23 & 22.78 & 50.34 \\
\hline PYRO TNT & $1 / 8$ in -28 days & 32.94 & 49.51 & 41.23 & 11.72 & 49.51 \\
\hline PYRO TNT & $1 / 4$ in - 7 days & 88.98 & 71.78 & 80.38 & 12.16 & 88.98 \\
\hline PYRO TNT & $1 / 4$ in -14 days & 95.71 & 52.41 & 74.06 & 30.62 & 95.71 \\
\hline PYRO TNT & $1 / 4$ in -28 days & 54.59 & 21.13 & 37.86 & 23.66 & 54.59 \\
\hline PYRO TNT & Averages & & & 65.34 & 37.96 & 92.19 \\
\hline PYRO SNT & $1 / 8$ in -7 days & 159.31 & 265.17 & 212.24 & 74.85 & 265.17 \\
\hline PYRO SNT & $1 / 8$ in -14 days & 144.50 & 81.42 & 112.96 & 44.61 & 144.50 \\
\hline PYRO SNT & $1 / 8$ in -28 days & 36.88 & 406.87 & 221.87 & 261.62 & 406.87 \\
\hline PYRO SNT & $1 / 4$ in -7 days & 98.51 & 68.21 & 83.36 & 21.42 & 98.51 \\
\hline PYRO SNT & $1 / 4$ in -14 days & 165.16 & 89.86 & 127.51 & 53.25 & 165.16 \\
\hline PYRO SNT & $1 / 4$ in - 28 days & 213.27 & 58.42 & 135.85 & 109.50 & 213.27 \\
\hline PYRO SNT & Averages & & & 148.96 & 94.21 & 215.58 \\
\hline EAGLE AIR & $1 / 8$ in -7 days & 267.65 & 318.82 & 293.24 & 36.18 & 318.82 \\
\hline EAGLE AIR & $1 / 8$ in -14 days & 237.82 & 378.59 & 308.21 & 99.54 & 378.59 \\
\hline EAGLE AIR & $1 / 8$ in -28 days & 31.28 & 334.98 & 183.13 & 214.75 & 334.98 \\
\hline EAGLE AIR & $1 / 4$ in - 7 days & 119.79 & 144.13 & 131.96 & 17.21 & 144.13 \\
\hline EAGLE AIR & $1 / 4$ in -14 days & Broken & 147.19 & 147.19 & & 147.19 \\
\hline EAGLE AIR & $1 / 4$ in -28 days & 161.17 & 83.38 & 122.28 & 55.01 & 161.17 \\
\hline EAGLE AIR & Averages & & & 197.67 & 84.54 & 247.48 \\
\hline RITE-CRETE & $1 / 8$ in -7 days & 12.43 & 20.61 & 16.52 & 5.79 & 20.61 \\
\hline RITE-CRETE & $1 / 8$ in -14 days & 18.44 & 0.00 & 9.22 & 13.04 & 18.44 \\
\hline RITE-CRETE & $1 / 8$ in -28 days & 13.88 & 9.53 & 11.70 & 3.08 & 13.88 \\
\hline RITE-CRETE & $1 / 4$ in - 7 days & 12.27 & 15.23 & 13.75 & 2.09 & 15.23 \\
\hline RITE-CRETE & $1 / 4$ in -14 days & No Sa & les -- Broke & moving & & \\
\hline RITE-CRETE & $1 / 4$ in - 28 days & 11.60 & 9.53 & 10.57 & 1.46 & 11.60 \\
\hline RITE-CRETE & Averages & & & 12.35 & 5.09 & 15.95 \\
\hline B-BOND & $1 / 8$ in -7 days & 121.81 & BROKEN & 121.81 & & 121.81 \\
\hline B-BOND & $1 / 8$ in -14 days & 83.07 & 53.45 & 68.26 & 20.95 & 83.07 \\
\hline B-BOND & $1 / 8$ in -28 days & 43.09 & 41.12 & 42.11 & 1.39 & 43.09 \\
\hline B-BOND & $1 / 4$ in - 7 days & 56.50 & 57.59 & 57.05 & 0.77 & 57.59 \\
\hline B-BOND & $1 / 4$ in -14 days & 91.36 & 72.92 & 82.14 & 13.04 & 91.36 \\
\hline B-BOND & $1 / 4$ in - 28 days & 33.87 & 92.19 & 63.03 & 41.24 & 92.19 \\
\hline B-BOND & Averages & & & 72.40 & 15.48 & 81.52 \\
\hline
\end{tabular}


The results and observations are summarized as follows.

1. The calculated average tensile strength of the eight sealants evaluated in this study ranged from 12 to 198 psi.

2. The results were less consistent than expected. It was expected that the load would correlate well with the thickness of the sealant, one of the control parameters in the tests. However, this was not the case. A contributing factor to the variability in the data was that the sealant seeped into the joint (figure 3-9) to differing degrees during the application. Although the thickness of the sealant relative to the face of the block was controlled, the effective thickness of the sealant at the joint was sometimes larger than the control measure. Since the sealant infiltration into the joint was inconsistent, this contributed to the inconsistency in the test results.
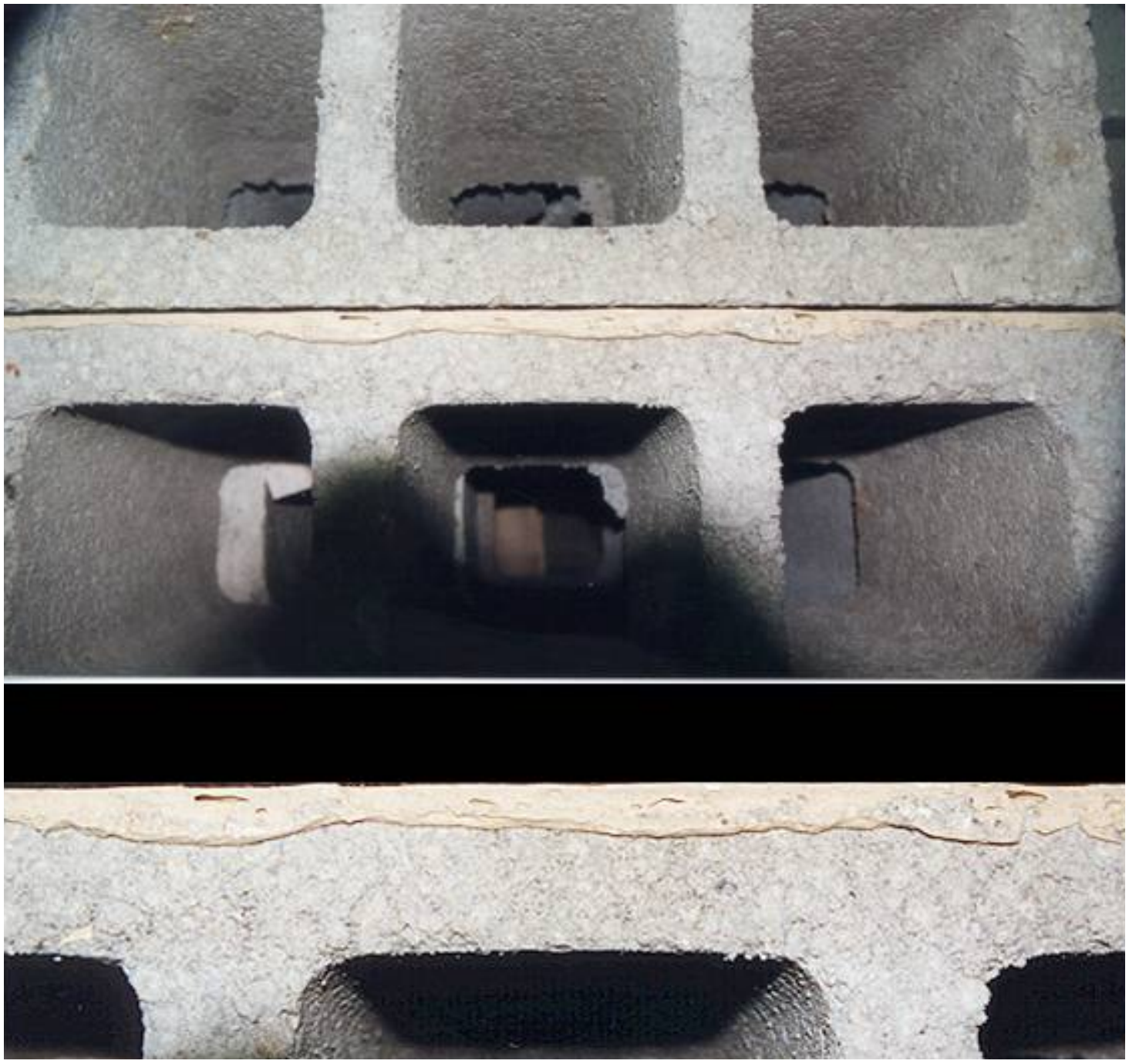

Figure 3-9. Sealant seeped into the block joint increasing the apparent thickness of the sealant. 
3. Although the loads were generally greater with the increasing sealant thickness, the increase in loading was disproportionate to the additional cross-sectional area of the sealant application. This is due, in large part, to the curing process where the thicker sealant was not fully cured throughout the sealant thickness in many specimens. Another possibility is that the outer fibers of the sealant are under higher stress as the joint opening develops, causing the outer fibers to fail first and then progress inward. Such analysis was beyond the scope of this study.

4. Inconsistency was also seen relative to the cure time. It was expected that all the sealants would increase in strength with cure time. Again, this was not the case. Several sealants actually decreased in strength as the cure time increased. In these cases, it appeared that the outer coating of the sealant cured, but the inner area was not hardened, let alone completely cured. This led to an apparent decrease in strength as the cured material, in these cases, was actually less, despite a thicker sealant application. One sealant was not fully cured even after 28 days. These inconsistencies in curing also contributed significantly to the inconsistency in the test results.

The applied loading can also be normalized to the area of the wall. To provide a measure commensurate with the CFR ASTM E 72 standard, the results of the two-block tests are extrapolated to a $4 \times 8-\mathrm{ft}$ section of wall. This is accomplished using the static analysis shown in figure 3-7, solving for the applied transverse force while using the same tensile strength computed from the two-block study. In other words, the assumption is made that the tensile strength of the sealant is a constant, and the applied loading for a normalized width of wall required to fail the sealed joint will be governed by the thickness and height of the wall. The transverse load is inversely proportional to the wall height and directly proportional to the wall thickness. Figure 3-10 shows the computed transverse load of a 6 - and 8 -in-thick, $4 \times 8$-ft wall using the average tensile strength computed from the two-block study. As shown in figure 3-10, the average extrapolated transverse load for a 6-in-thick wall ranged from 1 to $26 \mathrm{psf}$ and 2 to $42 \mathrm{psf}$ for the 8-in-thick walls. ASTM E 72 test data of $4 \times 8$ ft-sections of walls from 33 tests that were approved by MSHA using these same sealants, produced transverse loads for 8inch thick walls ranging from 42 to 129 psf with an average of 68 psf. Although the 
extrapolations from the two-block laboratory study were lower, this difference may be explained in the assumptions made regarding the thickness of the sealant. As previously noted, a sealant thickness of only $1 / 8$ in was assumed in this analysis, and the actual thickness of the sealant was likely larger than this, especially considering the fact that the sealant seeps into the joints of the block layers. The weight of the block is also ignored in this analysis, which as shown in Appendix A can contribute up to $7 \mathrm{psf}$ of transverse load capability to a dry-stacked stopping.

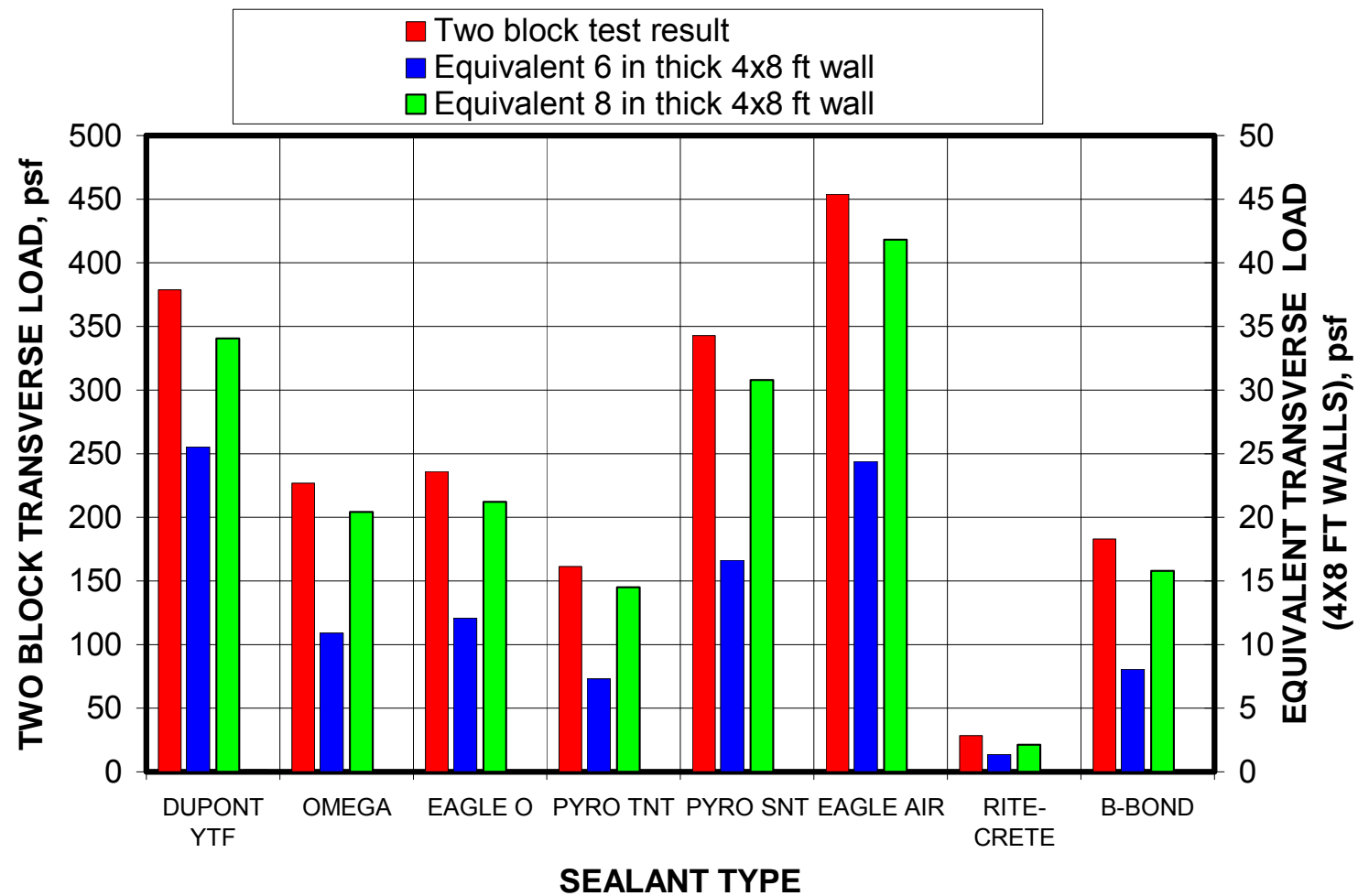

Figure 3-10. Computed transverse load for a $4 \times 8 \mathrm{ft}$ section of wall based on extrapolations of the two-block laboratory study.

\subsection{COMPARISONS TO CONVENTIONAL MASONRY DESIGN PRACTICES}

If the joints were mortared, a conventional flexural analysis could be used to analyze the wall behavior for transverse loading. The transverse load capacity of the wall would be governed by the tensile stress across the joint at the mid-span of the wall. The static analysis of this condition is expressed in equation 3.1. Using equation 3.1 and solving for the tensile strength $\left(f_{\text {tn }}\right)$ with a transverse load $(\mathrm{P})$ equal to $39 \mathrm{psf}$, the calculated tensile stress required to meet the minimum CFR transverse load requirement 
is approximately 50 psi for a 6-in-thick wall and 25 psi for an 8-in-thick wall. Comparing this to the average ASTM E 72 test data from MHSA, which yielded 68 psf for an 8-in-thick wall, the tensile strength of the mortared joint, would have to be approximately $47 \mathrm{psi}$. This shows that a mortared wall construction would be more effective than relying on the sealant for providing the tension to resist the moment induced by the transverse load in a free-standing wall application. However, dry stacked construction because of reduced labor costs is the most common form of stopping design employed in the United States.

$$
P=S \times\left(f_{t n}+\frac{f_{a}}{2}\right) \times \frac{8}{h^{2}}
$$

$$
\text { Where } \quad \begin{aligned}
P & =\text { transverse load, psf, } \\
\mathrm{S} & =\text { section modulus per unit width, in }{ }^{3} / \mathrm{in}, \\
f_{\mathrm{tn}} & =\text { tensile strength of block material, psi, } \\
f_{\mathrm{a}} & =\text { stress at base of wall caused by weight of block, psi, and } \\
\mathrm{h} & =\text { height of wall, ft. }
\end{aligned}
$$

In conclusion, the current CFR requirements using ASTM E 72 specifications for evaluating stopping walls is nothing more than a test of the sealant tensile strength (figure 3-11). Observations made in this research, and those reported by MSHA in the approval and certification of stoppings, indicate that inconsistent results can be achieved with these sealant-related test procedures. As described above, the test procedure is predicated on a freestanding wall arrangement, which for dry-stacked stopping constructions requires the sealant to control the transverse loading. The only other factor influencing the transverse loading capability is the height of the wall, and this factor is frequently ignored, as an 8-ft test height is a standard height used in ASTM E 72 testing. The physical and material properties of the block are irrelevant in this test procedure. This process will allow any block type to be used providing the sealant can sufficiently adhere to the block to provide the required tensile strength across the block joints. The next section will address a more realistic loading mechanism for stoppings constructed in a mine environment and further demonstrate the inadequacies of the current criteria. 


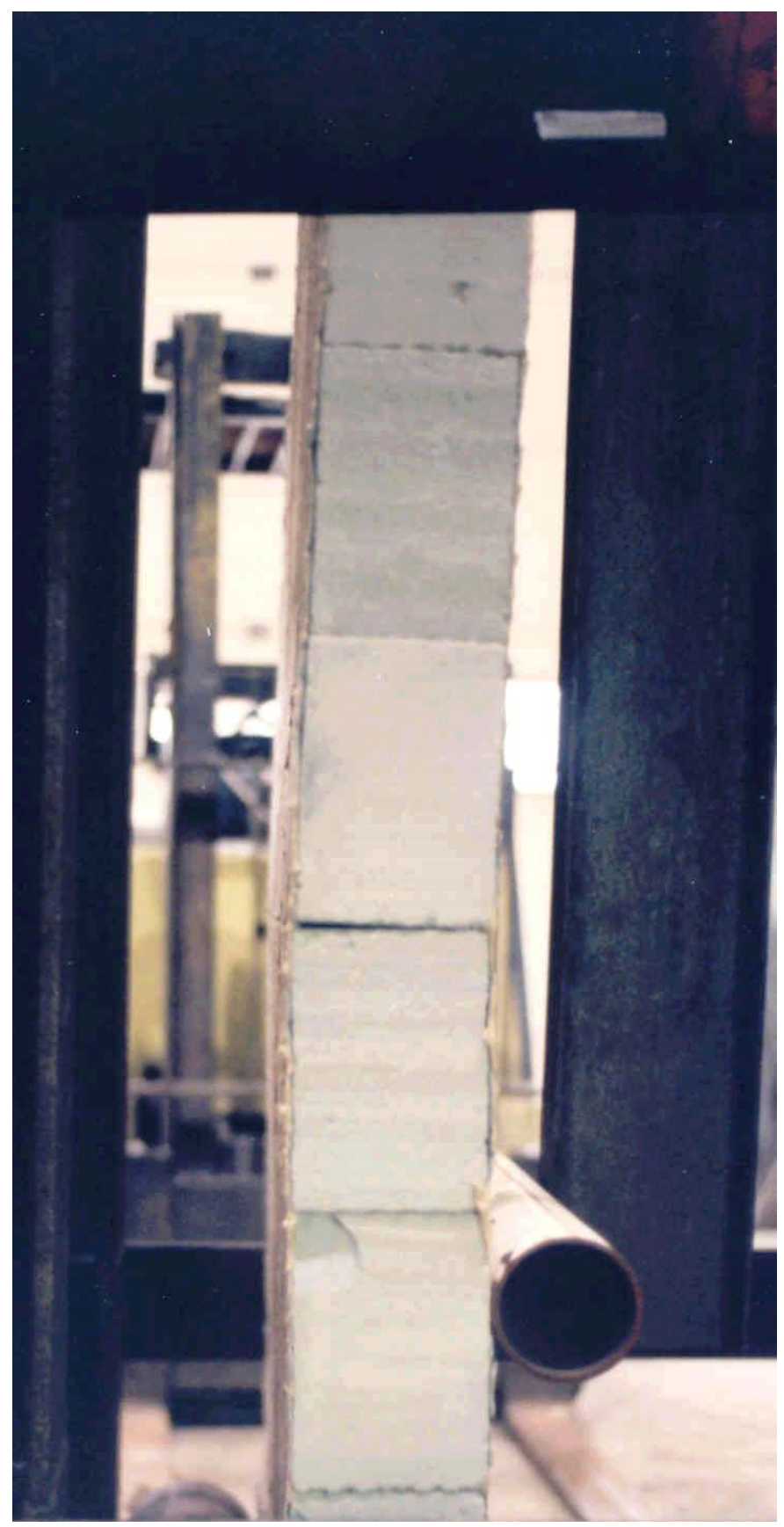

Figure 3-11. Failure of sealant on stopping wall during full-scale ASTM E 72 test. 


\section{CHAPTER 4 - RIGID ARCH LOADING MECHANISM}

In the mine, stopping walls are not freestanding structures as assumed in the ASTM E 72 test standard used to define the current CFR criteria. Stoppings, as constructed in the mine, bridge the distance between the mine floor and the mine roof and are typically wedged in place at the roof interface to provide a tight fit during installation. They also span the full entry width, butted against the pillars on both sides. Hence, if the mine stoppings are restrained by the mine roof and floor and pillars, this restraint allows for a completely different loading mechanism to occur, namely arching.

\subsection{PHYSICAL DESCRIPTION OF ARCHING}

Arching is the mechanism that occurs when the elongation of the tension face of the stopping due to the rotation of the wall as it bends under the application of transverse loading is prevented by the rigid contacts of the mine roof and floor. This arching of the wall produces a thrust that acts at the mine roof and floor interface, and produces compressive forces within the wall that can dramatically increase the transverse load capacity of the wall compared to a freestanding condition. An examination of the wall as it bends from the transverse loading further explains how arching works. Initially, the ends of the wall are in full contact with the mine roof and floor and the individual horizontal joints between the courses of block are in full contact with each other. As the transverse loading increases, the wall will begin to bend. Associated with the bending will be the opening of the joint along the mid height span of the wall (location of the maximum positive moment), and opening of the joints between the top and bottom block at the roof and floor interface (location of the maximum negative moment). A threehinged arch is formed where the external moment caused by the transverse loading ( $\left.\rho \times \mathrm{L}^{2} / 8\right)$ is resisted by the internal force couple $(\mathrm{P} \times \mathrm{r}$ ), where $\mathrm{r}$ is defined as the width of the arch and $\mathrm{P}$ is the thrust generated by the arching. This condition is illustrated in the diagram in figure 4-1, and expressed mathematically by equations 4.1 and 4.2. As shown in the figure, crush zones occur at the three hinge points. 


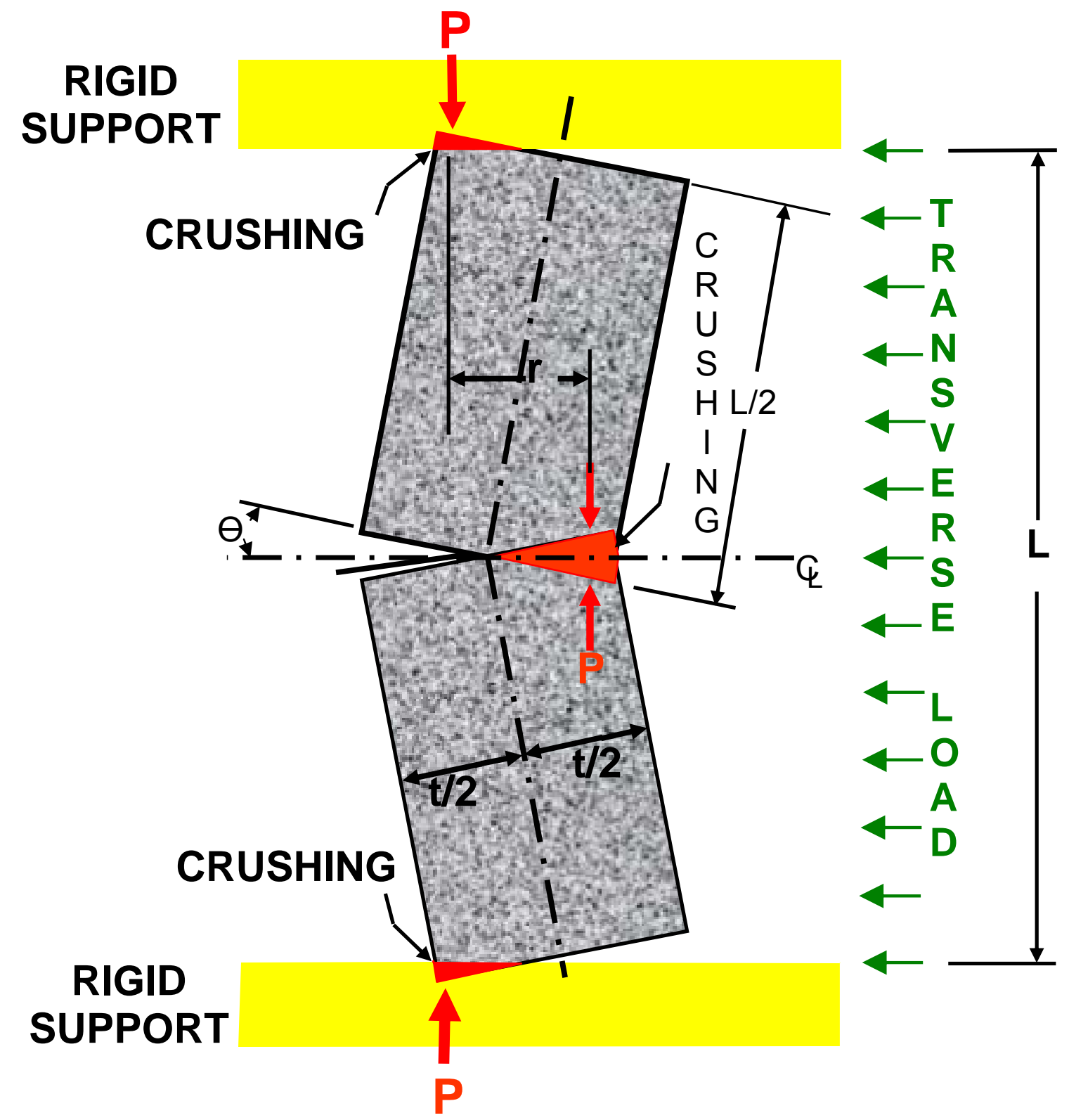

Figure 4-1. Illustration of rigid arching of a wall structure. 


$$
\begin{gathered}
\frac{\rho \times \mathrm{L}^{2}}{8}=\mathrm{P} \times \mathrm{r} \\
\rho=\frac{8 \times \mathrm{P} \times \mathrm{r}}{\mathrm{L}^{2}}
\end{gathered}
$$

Where $\rho=$ transverse load, psi,

$\mathrm{L}=$ height of the wall, in,

$\mathrm{P}=$ resultant thrust force at the hinge points, lbs per in of wall width, and $r=$ width of the arch, in.

In this analysis, the compressive forces will control the transverse load capacity. Hence, the compressive strength of the block material becomes the dominant control parameter in defining the transverse load capacity. Lower strength blocks will have less transverse load capacity than higher strength blocks. This is a significant departure from the dominance of the sealant in controlling the transverse load capacity in the current CFR testing requirement. Under arching conditions, the contribution of the sealant to the transverse load capacity would be insignificant for all but the very weakest block materials used for stopping construction.

\subsection{THEORETICAL ASSESSMENT OF RIGID ARCHING}

A free-body diagram of a stopping wall in an arching condition is shown in figure 4-2. In this simplistic analysis, a "zero" contact area is assumed (the resultant thrust force (P) is shown to act at the very edge of the wall), making the width of the arch (r) equal to the wall thickness (t). However, as shown in figure 4-1, crush zones develop at the hinge points. The extent of the crush zones will depend on the stress-strain properties of the material and the deflection of the wall (Cranston and Roberts, 1976). The analysis illustrated in figure 4-3 assumes the contact area is one tenth of the block thickness $(0.1 \mathrm{t})$ and uniform loading distribution acting on this area. Using these assumptions, the width of the pressure arch is then equal to $0.9 \mathrm{t}$. If it is assumed that the 
arching thrust $(\mathrm{P})$ is controlled by the compressive strength of the material, an expression for $\mathrm{P}$ can be derived as shown in equation 4.3. Substituting the expression for $\mathrm{P}$ from equation 4.3 into equation 4.2, yields a solution for determining the transverse load capacity of a stopping wall (equation 4.4).
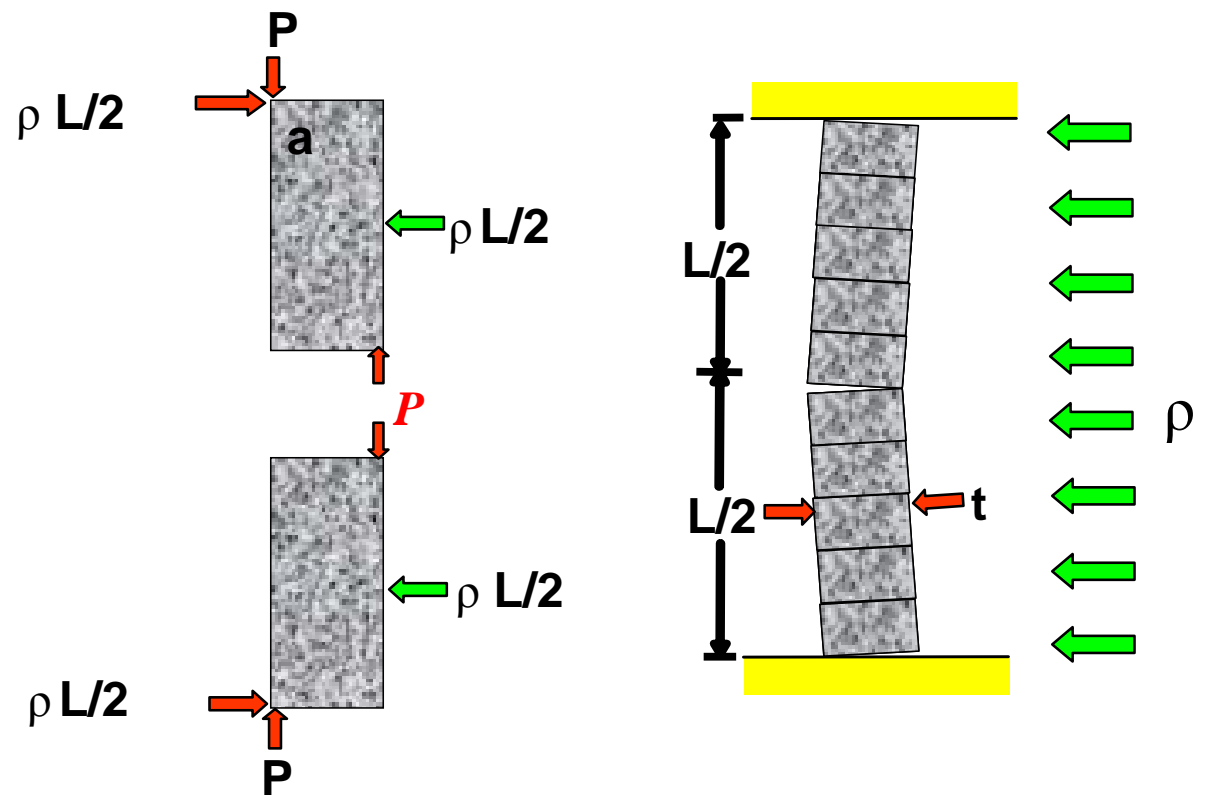

Static Analysis:

$\rho=$ Transverse loading, $\mathrm{psf}$

$\Sigma \mathrm{M} @ \mathrm{a}=0$

$P=$ Arching thrust, Ibs/unit

$\rho(L / 2)(L / 4)=P(t)$ width of wall,

$\mathrm{L}=$ Height of wall, $\mathrm{ft}$

$\rho=\frac{8 \mathrm{Pt}}{\mathrm{L}^{2}}$

$\mathrm{t}=$ Thickness of wall, $\mathrm{ft}$

Figure 4-2. Analysis for rigid arching of a wall structure.

$$
P=0.1 \times t \times f_{c}
$$

Where $P=$ arching thrust, lbs per in of wall width,

$\mathrm{t}=$ thickness of wall, in, and

$f_{\mathrm{c}}=$ compressive strength, psi 


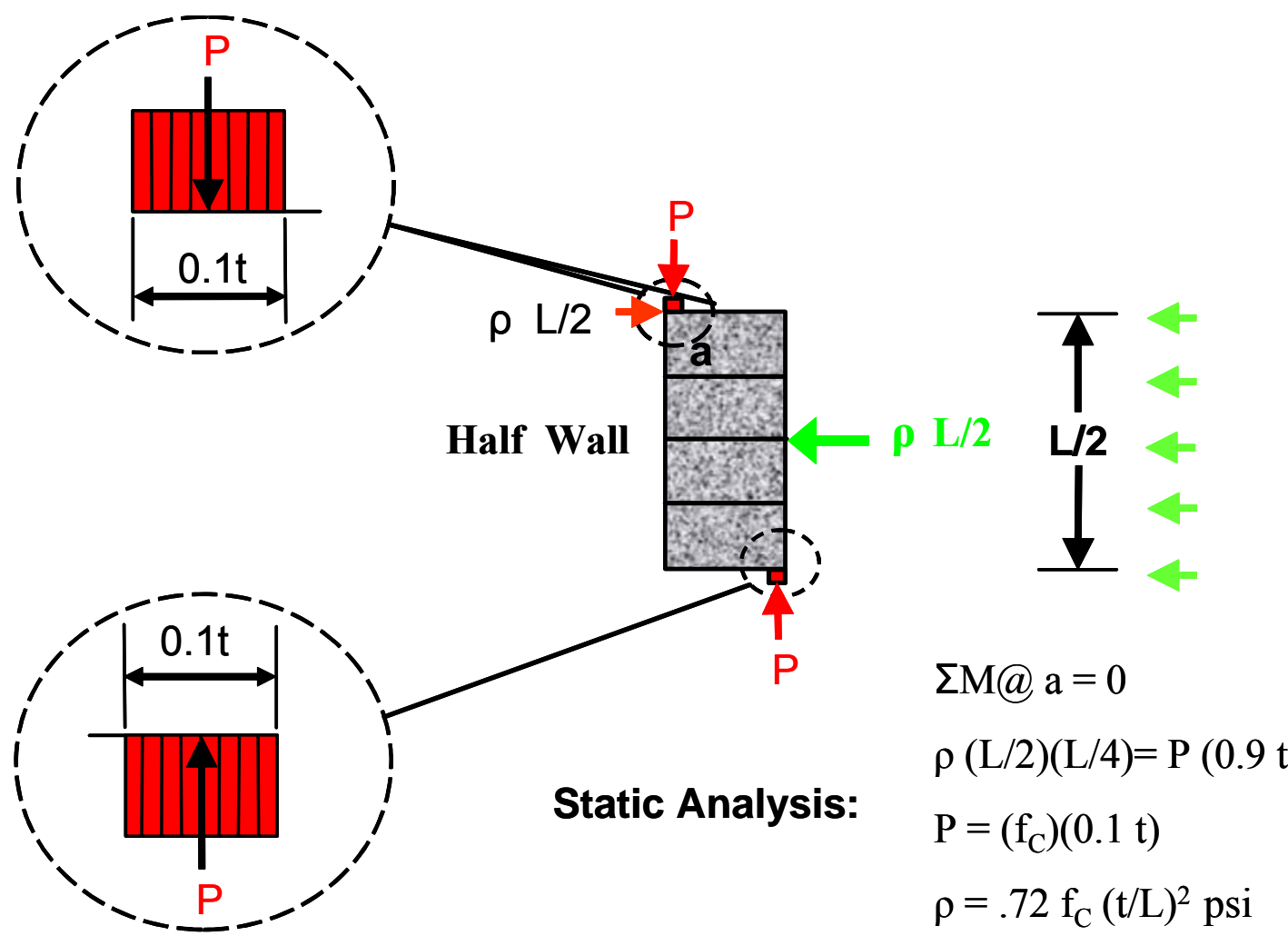

Figure 4-3. Examination of the rigid-arching loading conditions and expressing the transverse loading as a function of the block compressive strength.

$$
\rho=0.72 \times f_{c} \times\left(\frac{t}{L}\right)^{2}
$$

Where

$$
\begin{aligned}
& \rho=\text { transverse load, psi, } \\
& L=\text { height of the wall, in, } \\
& t \quad=\text { thickness of wall, in, and } \\
& f_{c}=\text { compressive strength, psi. }
\end{aligned}
$$

From these equations, it is seen that in addition to the compressive strength of the block, which limits the thrust force within the wall, the transverse load is also directly related to the width of the pressure arch and inversely related to the square of the wall height. The width of the pressure arch depends on, among other things, the thickness of the wall or 
block thickness. Thicker walls will have significantly greater transverse load capacity than thinner walls, all other things being equal. Therefore, for blocks that are anisotropic, the orientation of the block (wide-side-down or wide-side-up) during wall construction can have a significant impact on the transverse load capacity. Likewise, an increase in the roof-to-floor height of the wall of just a foot can decrease the transverse load capacity of a wall by 20 to 30 pct.

\subsection{TRANSVERSE LOAD DESIGN EQUATION}

Multiplying equation 4.4 by 144 allows the normalized transverse load $(\rho)$ to be computed in units of pounds per square foot (equation 4.5) as a function of the wall thickness $(\mathrm{t})$, height $(\mathrm{L})$, and compressive strength $\left(f_{\mathrm{c}}\right)$.

$$
\rho=103.68 \times f_{c} \times\left(\frac{t}{L}\right)^{2}
$$

$$
\text { Where } \begin{aligned}
\rho & =\text { transverse load, } p s f \\
\mathrm{t} & =\text { thickness of wall, in, } \\
\mathrm{L} & =\text { height of section of wall that arches, in, and } \\
\mathrm{f}_{\mathrm{c}} & =\text { compressive strength, psi. }
\end{aligned}
$$

This can be expressed graphically as shown in figure 4-4, which plots the predicted transverse load as a function of the term $f_{\mathrm{c}} \times(\mathrm{t} / \mathrm{L})^{2}$. Using this graph, the transverse load capacity of walls constructed from a specific material strength can be determined for different combinations of wall thickness and height. An example is also illustrated in figure 4-4. This particular example assumes a 6-in-thick wall that is 72 inches in height and constructed of concrete block with a compressive strength of 1,000 psi. The term $f_{\mathrm{c} x}(\mathrm{t} / \mathrm{L})^{2}$ equates to $6.94 \mathrm{psi}$ for this example. Locating this on the $\mathrm{x}$-axis and finding the intersecting point on the design curve, determines a predicted transverse load capacity of 720 psf. 


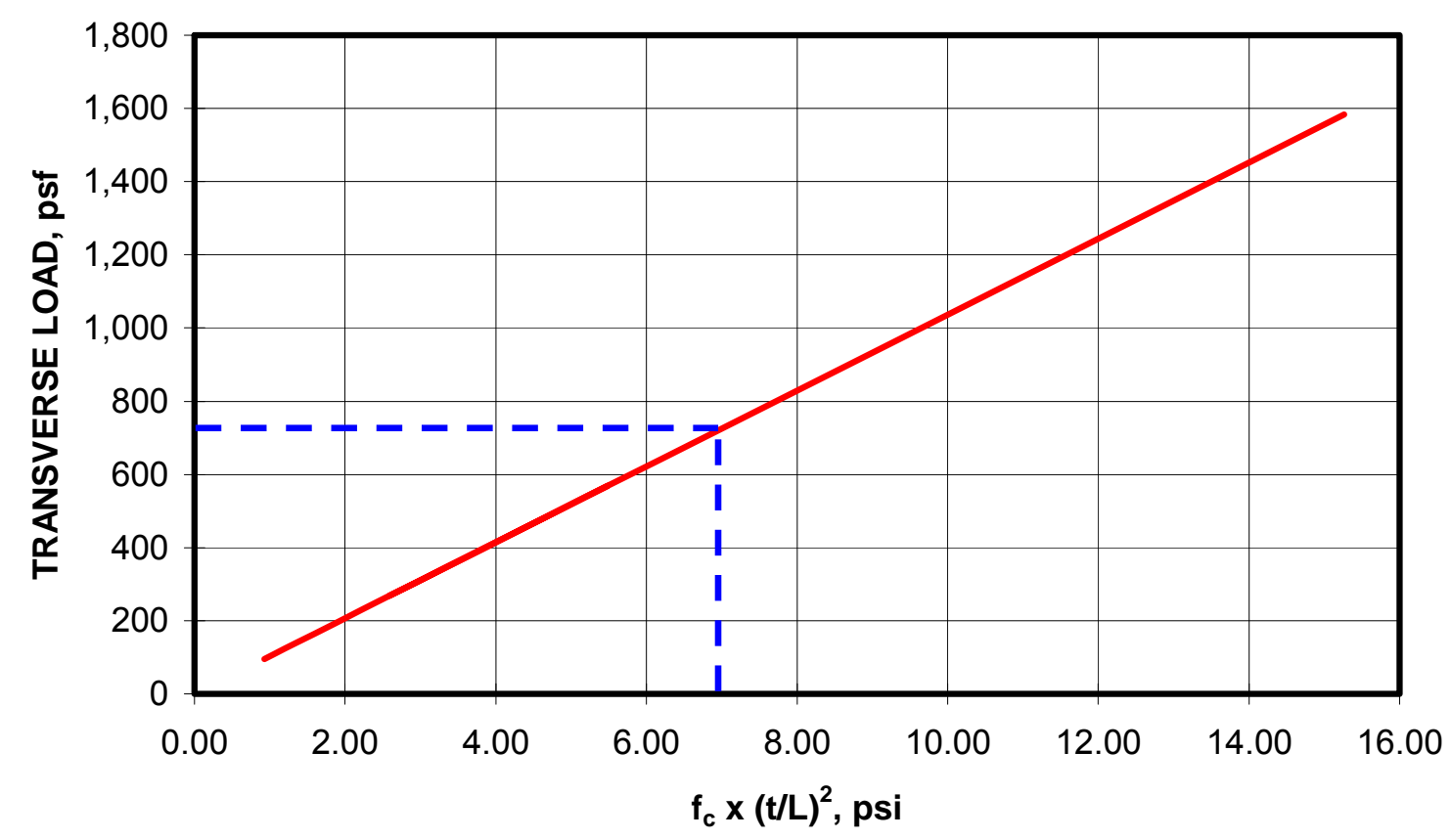

Figure 4-4. Graphical illustration of rigid-arching design equation with an example consisting of a 6-inch thick wall, 72 inches in height, with a material compressive strength of 1,000 psi.

\subsection{IMPLICATIONS OF RIGID ARCHING TO STOPPING DESIGN}

Rigid arching indicates that the physical properties of the block and the size of the mine opening must be considered in the design of a stopping. Intuitively, higher strength block will provide greater transverse load capability, but increasing the thickness of the block, or constructing the stopping with the wide side of the block as the contact area, can also greatly increase the transverse load capacity of the stopping. Likewise, it is important to recognize that the transverse load capacity will decrease as the entry height increases. These relationships are illustrated in figure 4-5 for conventional concrete masonry units that have historically been used to construct stoppings. These solid blocks measure nominally $6 \times 8 \times 16$ inches and have a unit block compressive strength of 1,330 psi. As seen in the figure, using the 8-in-wide construction nearly doubles the transverse load capacity of the stopping for a specific construction height. For comparison, a stopping constructed in a $16-\mathrm{ft}$-wide by 8 -ft-high opening, the wide-side construction would require 192 blocks while the narrow-side construction would require 144 blocks. In other words, for a 33 pct increase in the number of block, the transverse load capacity 
can be increased by 100 pct. On the other hand, the transverse load capacity will drop by a factor of four if the construction height is doubled.

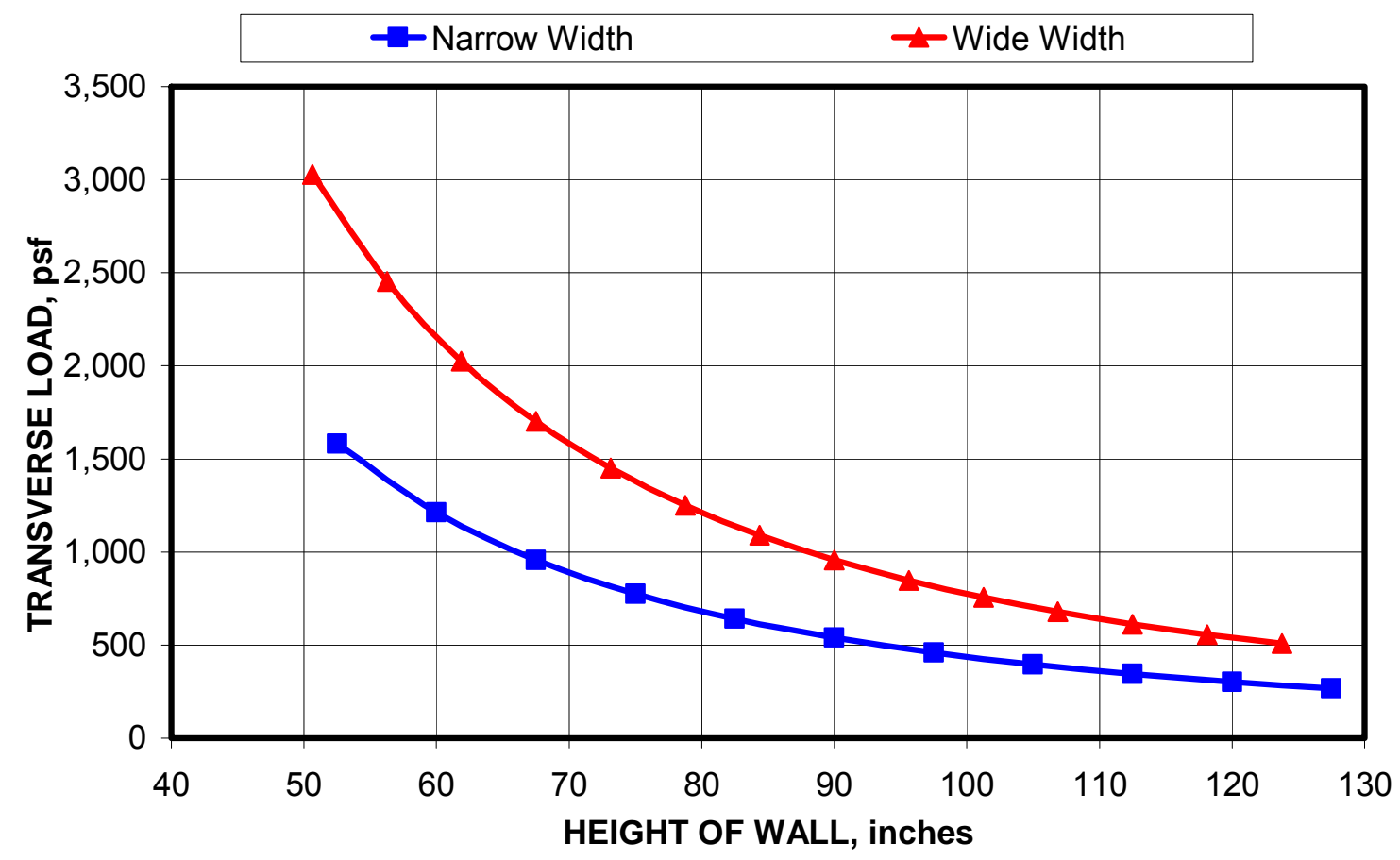

Figure 4-5. Impact of block orientation and wall height on transverse loading capacity for conventional concrete block used in stopping construction (1,330 psi compressive strength with 6x8x16 in nominal dimensions). 


\section{CHAPTER 5 - SIMULATING RIGID ARCHING THROUGH BIAXIAL LOADING IN THE MINE ROOF SIMULATOR}

It is apparent from the preceding analysis that an assessment of the true transverse load capacity of a mine ventilation stopping cannot be attained by a freestanding wall evaluation. Rigid arching has been shown to be an accepted loading mechanism for masonry design for walls that are restrained by rigid abutments. A stopping bridging between the mine roof and floor satisfies this condition. However, full-scale testing of mine ventilation stoppings in an actual underground mine is difficult and time consuming to conduct. Likewise, there are only a few facilities where full-scale laboratory tests can be conducted. Laboratory testing of partial masonry beams by other researchers have been successfully conducted, although these too have been relatively limited in scope, requiring specialized reaction frames and fixtures to accomplish rudimentary tests. As the theory indicates, the thrust forces involved in rigid arching of wall structures can be substantial (over 100 tons of abutment loading for a 4-ft wide wall). This requires robust fixtures to preserve the "rigid" abutment condition. NIOSH has a unique load frame that is designed to simulate the behavior of rock masses for underground mining operations. It is called the Mine Roof Simulator (MRS). This unique facility provides an ideal framework in which to conduct rigid-arch testing of stopping walls.

\subsection{DESCRIPTION OF THE MINE ROOF SIMULATOR}

A photograph of this unique machine is shown in figure 5-1. A detailed description of the load frame is provided in Appendix B. The platen size measures $20 \mathrm{x} 20 \mathrm{ft}$, and with a maximum vertical opening of $16 \mathrm{ft}$, the MRS can accommodate full-scale stopping constructions, as shown in figure 5-2. The MRS is capable of providing controlled biaxial loading in the vertical and one horizontal axis. Up to 3 million lbs of vertical force can be applied through a 24-in stroke of the lower platen and 1.6 million lbs of horizontal force through a 16-in stroke of the lower platen. The loads or displacements in these two axes can be applied individually or simultaneously if desired. 


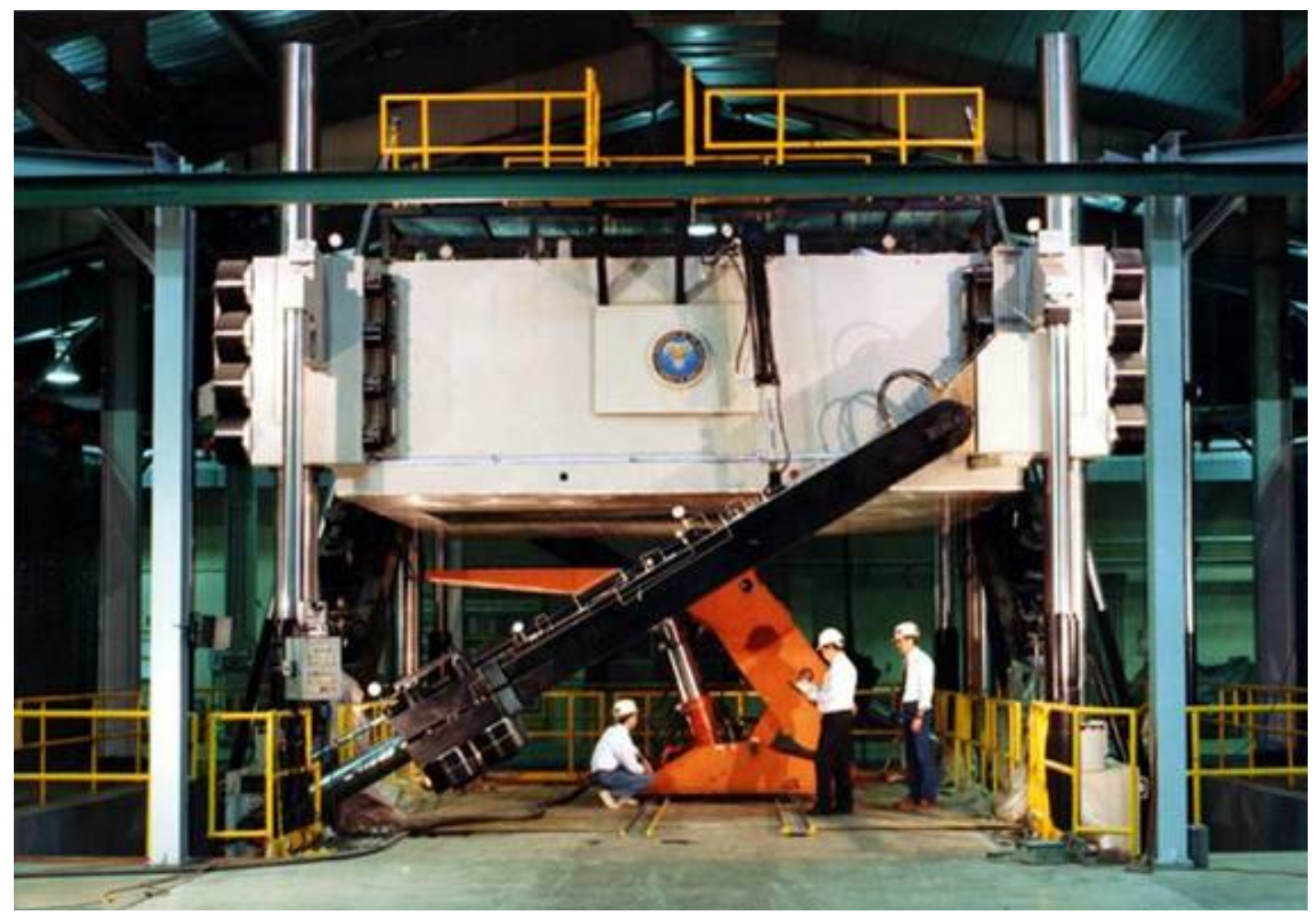

Figure 5-1. NIOSH Mine Roof Simulator load frame.

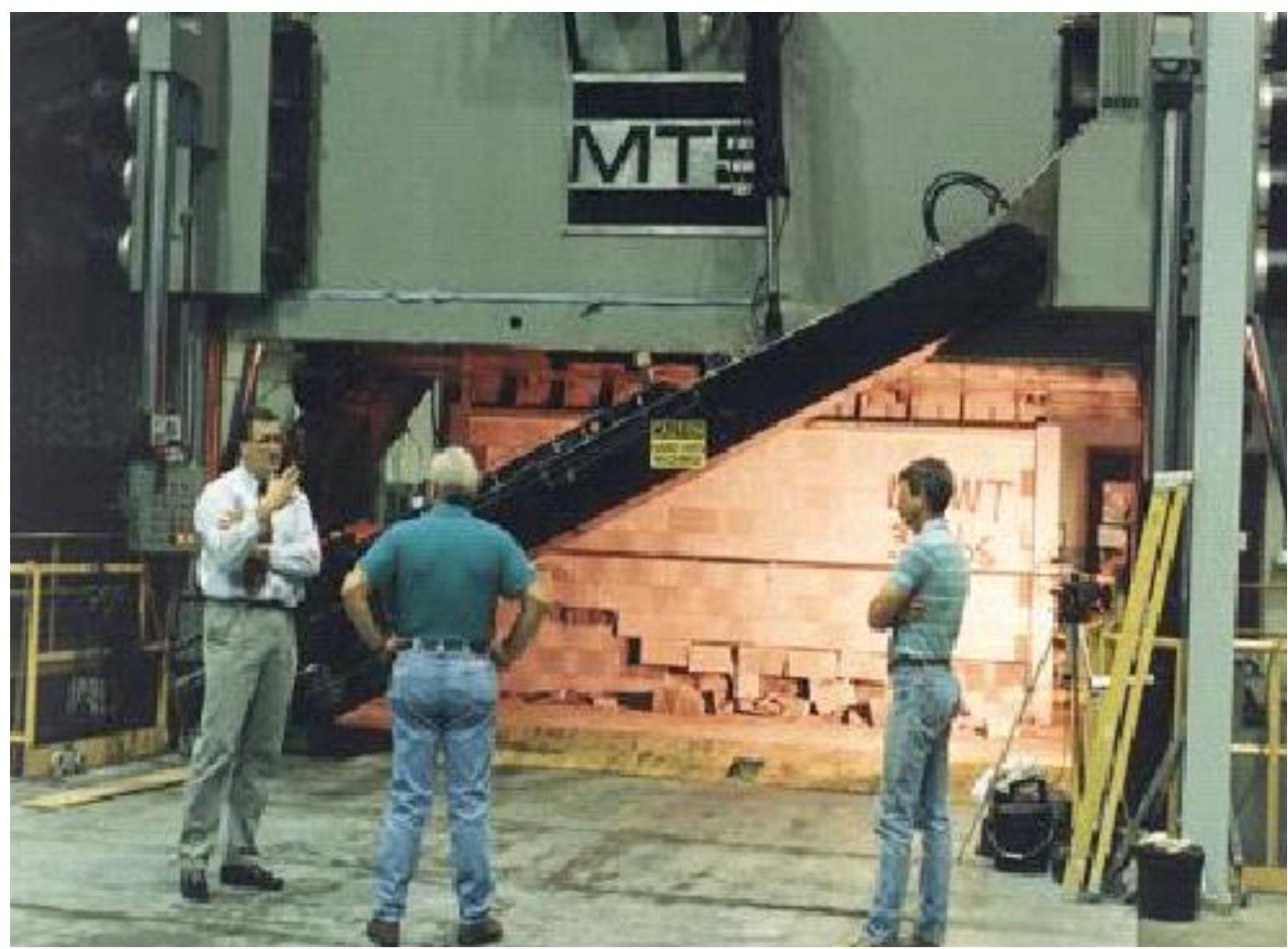

Figure 5-2. Full-scale mine ventilation stopping wall being tested in the NIOSH Mine Roof Simulator. 


\subsection{TEST PROTOCOL FOR SIMULATING RIGID ARCHING}

In order to simulate rigid arching, a half-height section of a stopping wall is placed in the load frame in a typical vertical orientation, as it would be in the mine. The upper platen position is adjusted to the height of the block column and is hydraulically clamped to maintain its position. The vertical position of the lower platen is commanded to remain constant. Hence, the fixed positions of the upper and lower platen allow them to act as rigid restraints. The lower platen is then moved horizontally at a constant velocity of 0.5 inches/minute, causing the wall to rotate (figure 5-3). As the base of the wall is forced to move horizontally, crush zones are created at the ends of the wall on opposite sides, consistent with the rigid-arch loading mechanism. The horizontal load applied by the MRS is measured and is equivalent to the transverse load acting on a stopping wall. This load, measured in pounds of force, is then normalized to the square foot area of the wall to determine the transverse load capacity of the wall in units that are comparable to the current CFR requirements.

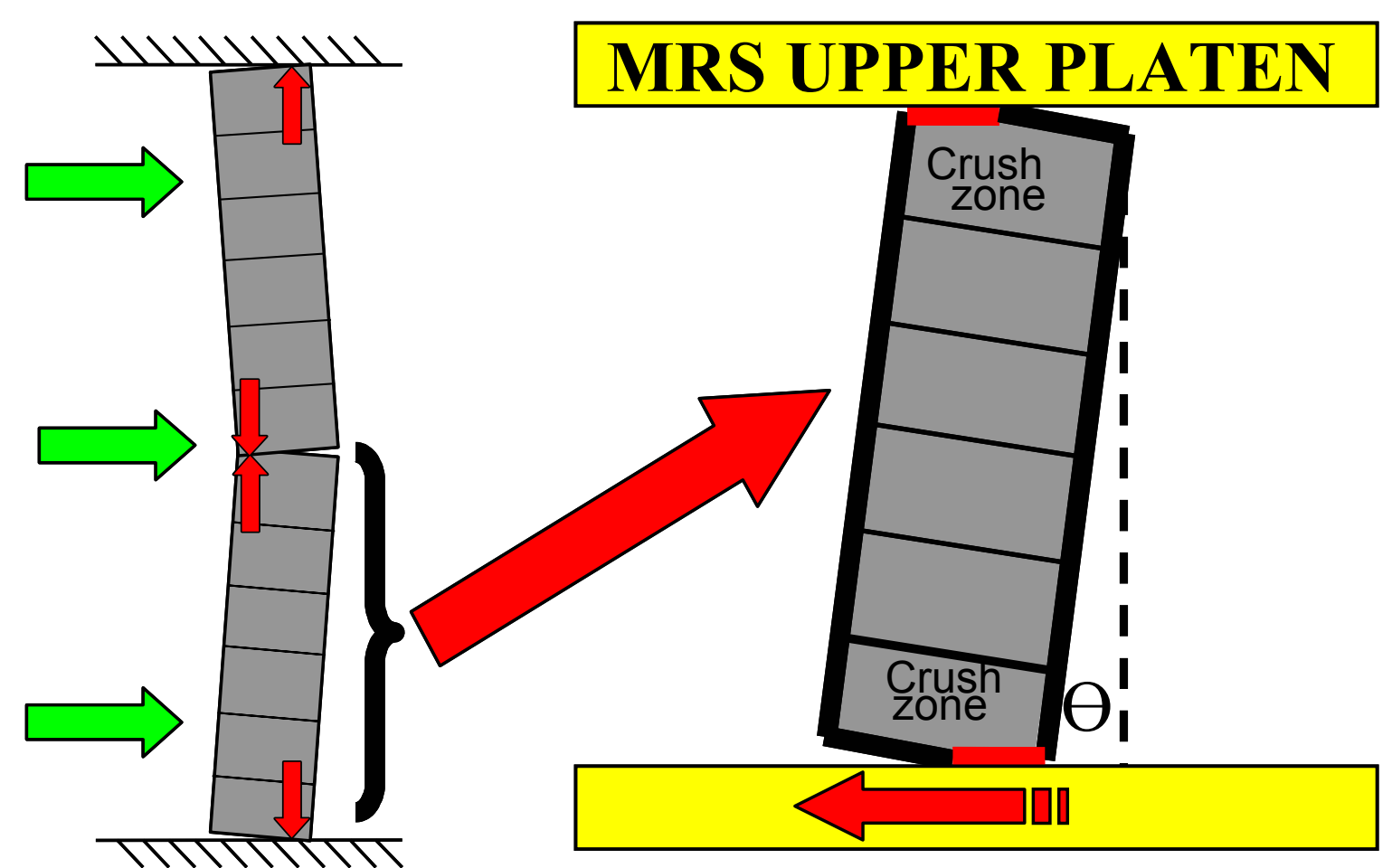

Figure 5-3. Diagram illustrating the simulation of rigid arching on a half-wall section of a stopping by biaxial testing in the NIOSH Mine Roof Simulator. 
In order toy measure the applied horizontal loading more accurately than the capabilities of the MRS, an arrangement using load cells was employed which can independently record the horizontal load at a higher resolution than the MRS. Figure 5-4 illustrates the testing apparatus. A single or triple column of block, equal in height to half the full-scale installation height is constructed on the rolling platform. The rolling platform is a two-in-thick steel plate that is secured to four 20-ton-capacity crawler units (figure 5-5). A load measuring reaction fixture is located adjacent to the crawler assembly. Two bolts are secured into the lower platen, which has inserts on a $20 \times 20$-in grid to accommodate bolt placements. The bolts serve as the rigid horizontal restraint against which transverse loading of the block column is generated. A two-in-thick metal plate is then used to bridge the gap between the two bolts. Two, 20-kip load cells are then placed in front of the reaction plate to accurately measure the horizontal load (figure 5-6). These have a calibration accuracy of $0.1 \mathrm{pct}$, meaning they can measure the transverse load to an accuracy of $20 \mathrm{lbs}$. The load cells laid horizontally on the platen have a threaded bar extending from them to provide contact with the stopping block's rolling platform. Two machined nuts at the end of the threaded bar provide some minor adjustments to ensure that proper contact is established with the block platform before the test commences.

Although the apparatus is designed to test a column up to three blocks wide, it was concluded from shakedown testing that a single column of block would provide the most consistent results. Block dimensional tolerances can cause variations in the height of the wall across a three-block arrangement (figure 5-7) for dry-stacked block constructions that are being evaluated in this study. Since the MRS is acting as a rigid restraint, any differential in the height of the wall will produce non-uniform loading of the wall from block-to-block across the width of the wall. By using a single column of block, a uniform height can be more easily achieved to provide uniform loading and results that are more consistent. 


\section{Upper Platen}

\section{SIDE VIEW}
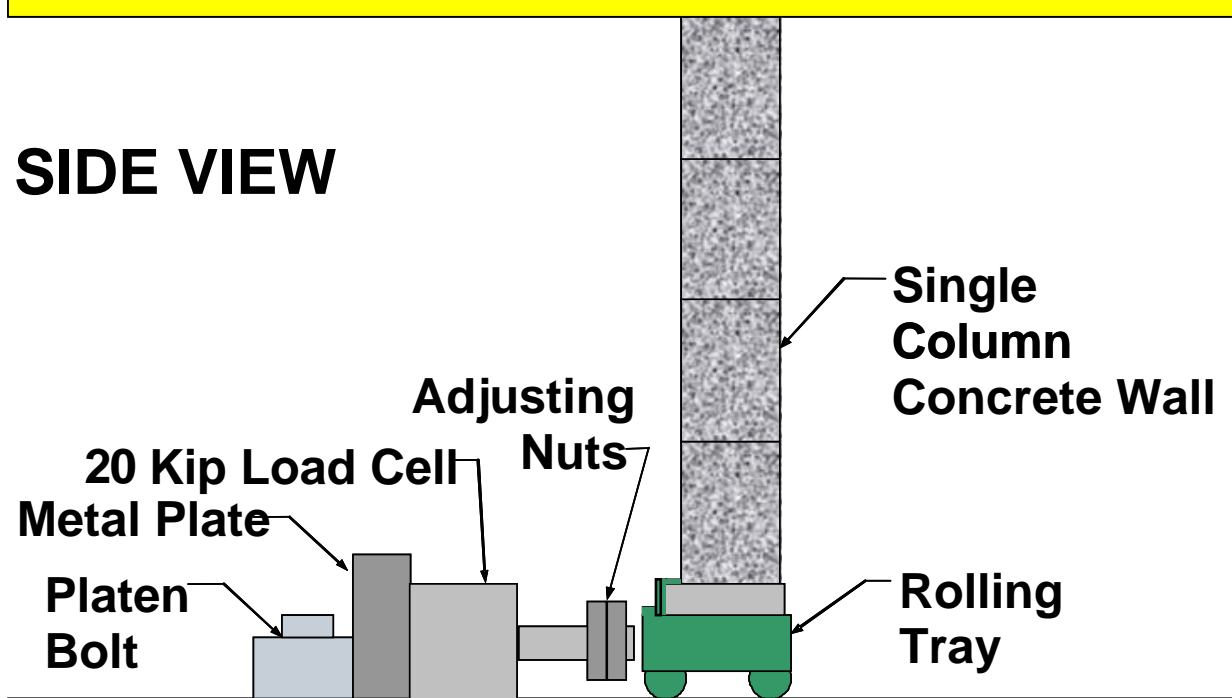

\section{Lower Platen}

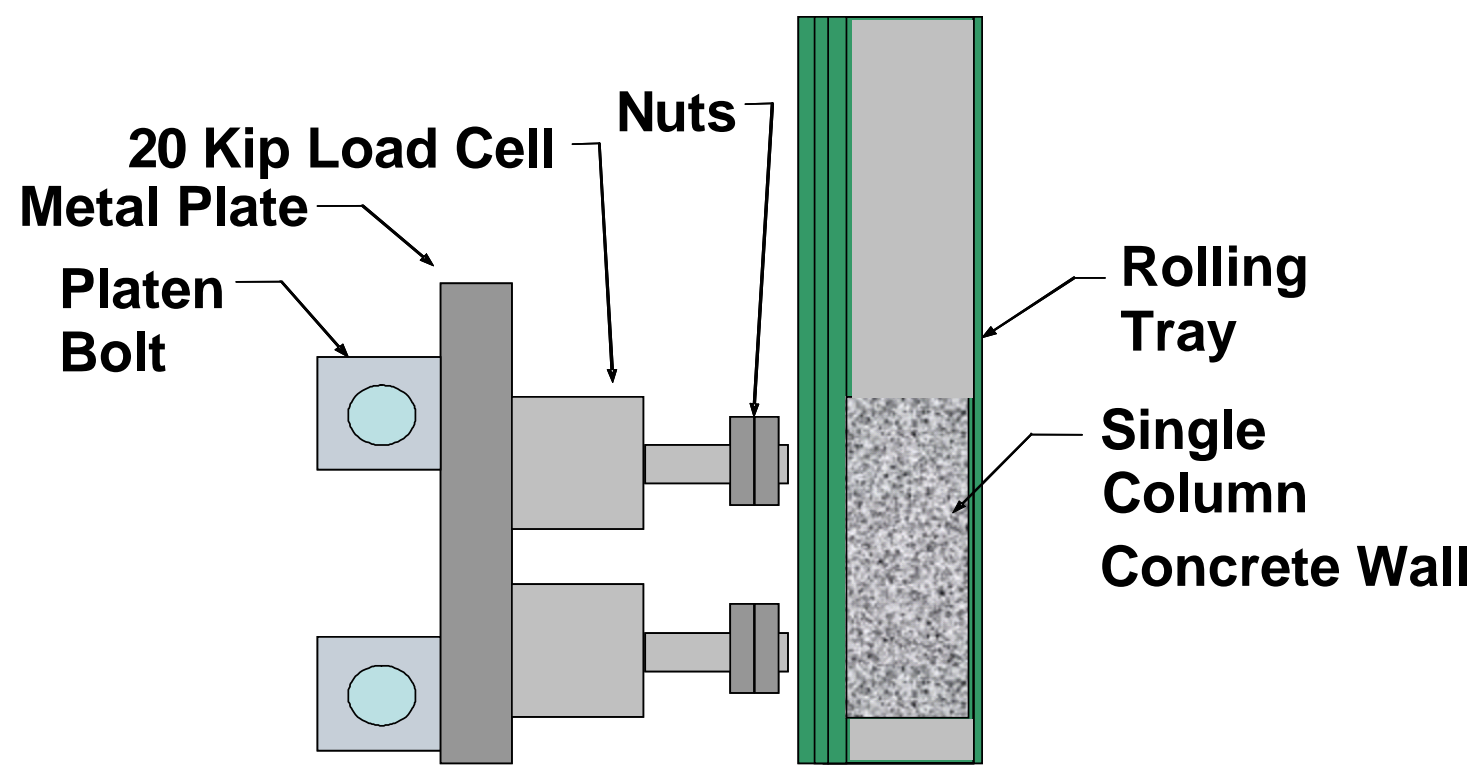

PLAN VIEW

Figure 5-4. Apparatus used to conduct half-wall rigid-arching tests of stopping walls in the NIOSH Mine Roof Simulator. 


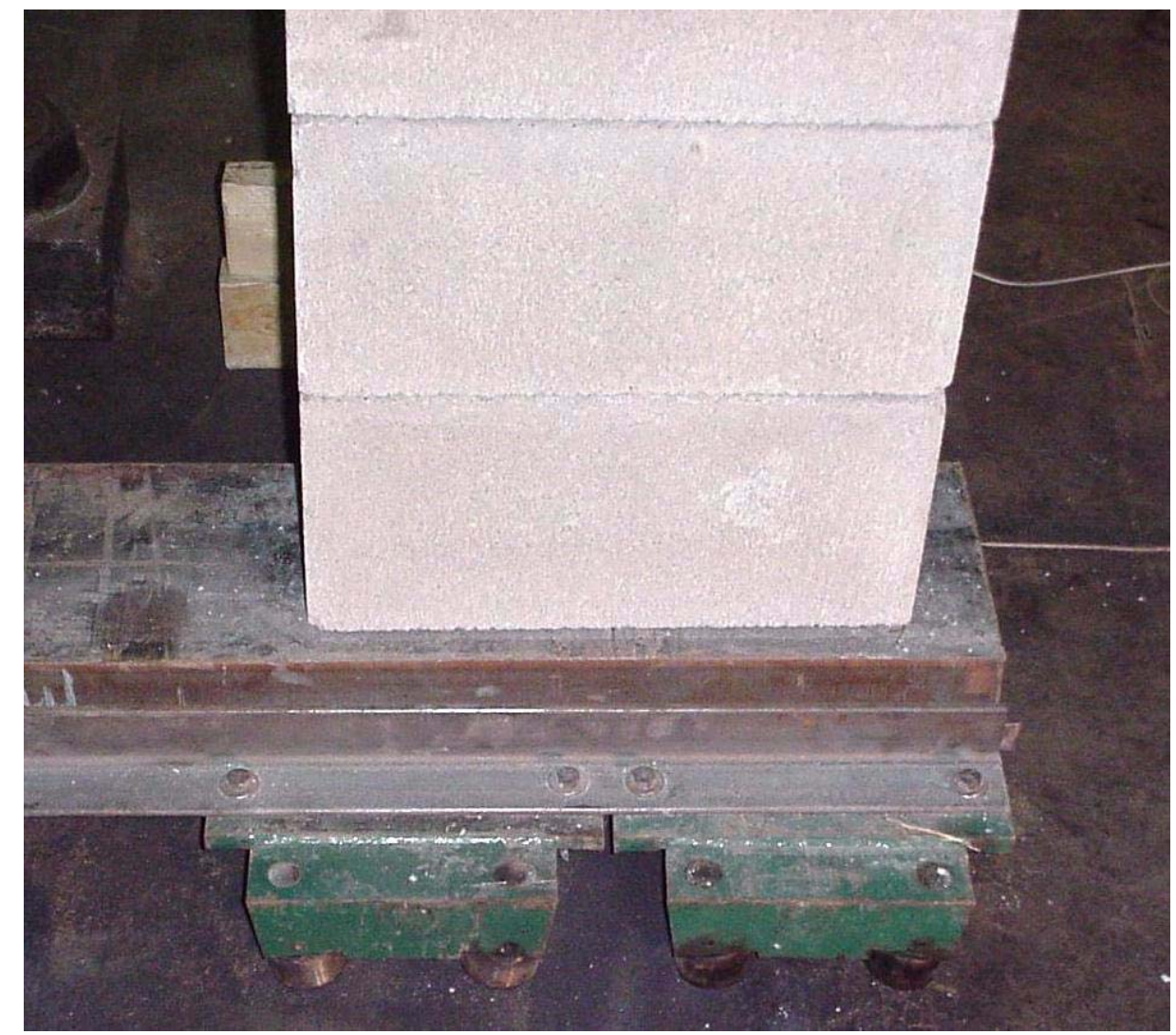

Figure 5-5. Block column rests on rolling platform to allow load cells to measure lateral loading.

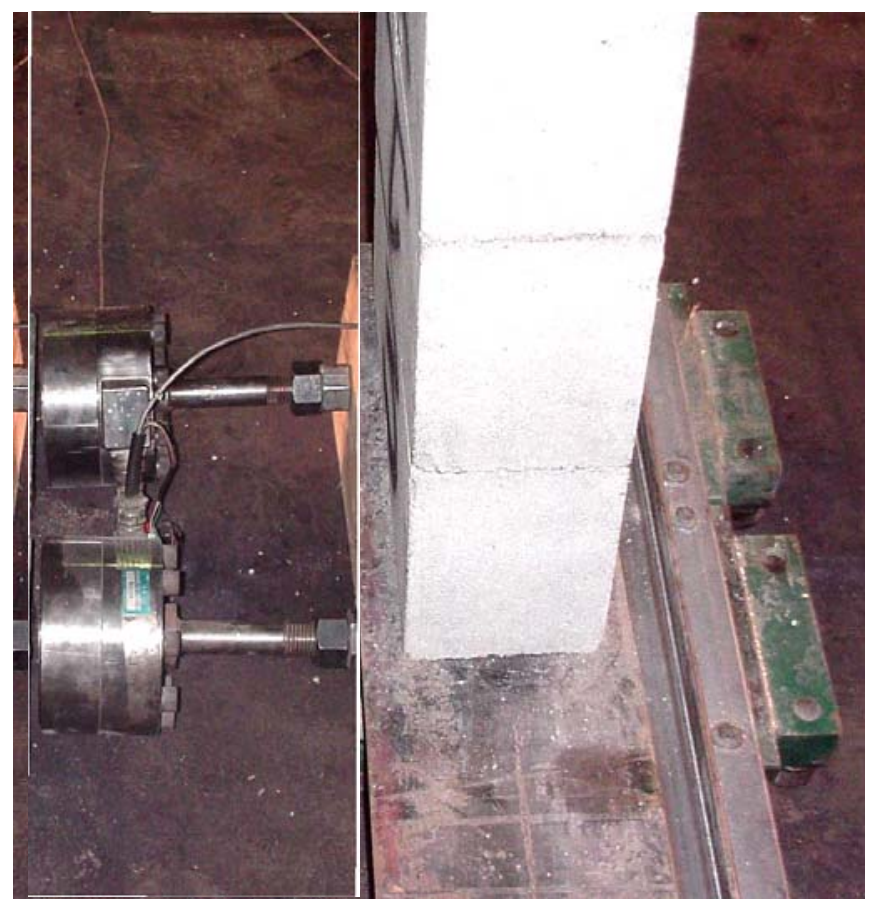

Figure 5-6. Load cells used to accurately measure lateral loading. 


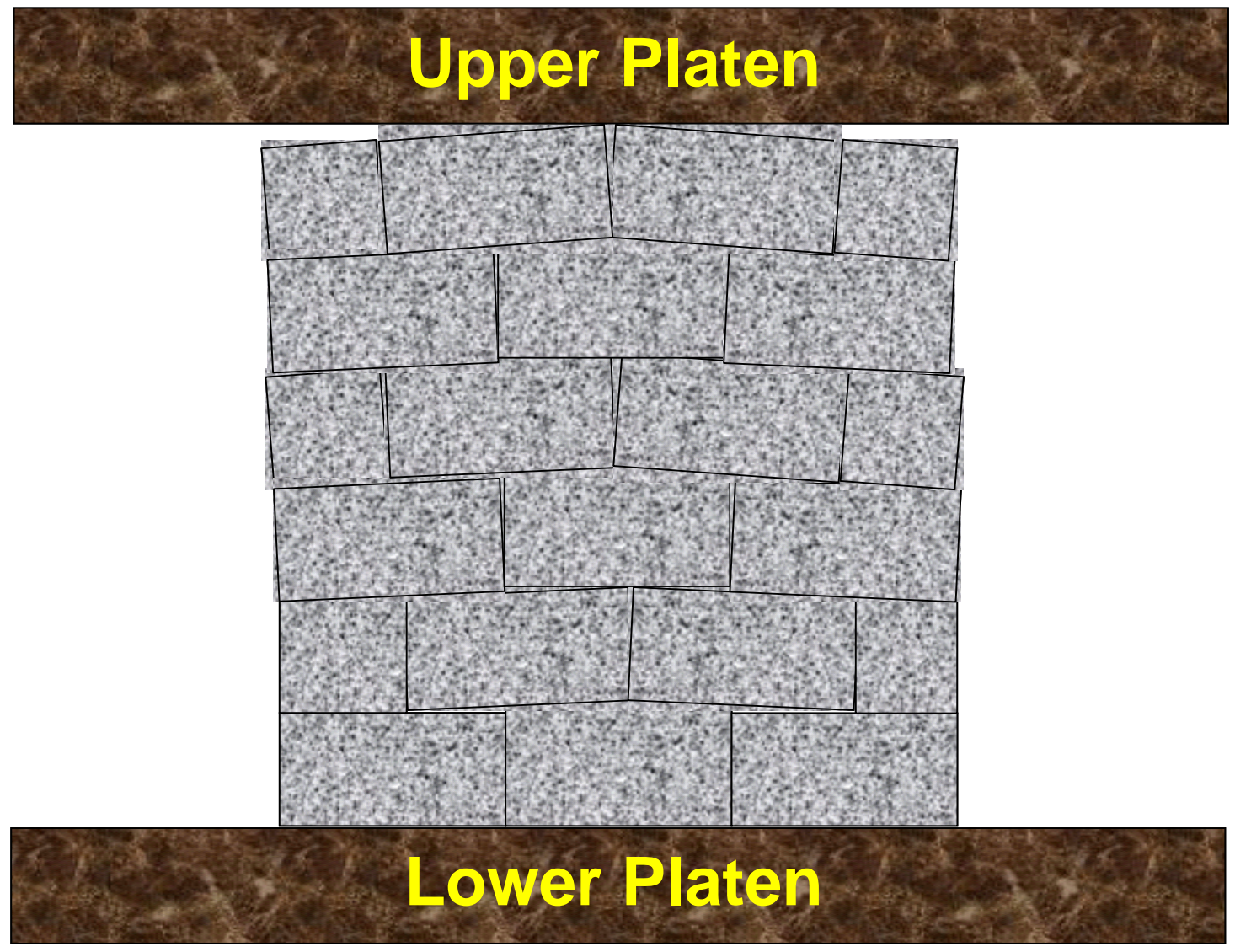

Figure 5-7. Illustration of how block dimensional tolerances can cause localized loading on a three-block-wide wall (not to scale).

\subsection{TRANSVERSE LOAD DETERMINATIONS FROM MRS HALF-WALL TESTING}

Two examples of transverse loading tests conducted on two different types of concrete block are shown in figures 5-8a and 5-8b. Both tests consisted of a single column of block, stacked four blocks high with the narrow side contact between blocks. The first test (figure 5-8a) is a half-wall constructed from a lightweight block manufactured by Kingsway Technology from autoclaved concrete. Air pockets introduced into the concrete mix (figure 5-9) result in the low material density. This block measures $5.875 \times 8.375 \times 17.250$ inches with a density of $42.5 \mathrm{lbs} / \mathrm{cu} \mathrm{ft}$ resulting in a unit block weight of approximately $21 \mathrm{lbs}$. Tests conducted on an individual block indicated that the compressive strength was 546 psi (figure 5-10). This type of block is being used by some mines because of its lighter weight to reduce material handling injuries associated with stopping construction. The second wall was constructed from 
block made by Klondike Block and Masonry Supplies, Inc., from conventional Portland cement, sand, and aggregate material. This block measured $5.625 \times 7.500 \times 15.625$ inches with a material density of $109.7 \mathrm{lbs} / \mathrm{cu} \mathrm{ft}$ and a unit weight of approximately 45 lbs. This block has a compressive strength of 1,330 psi as shown in figure 5-11.
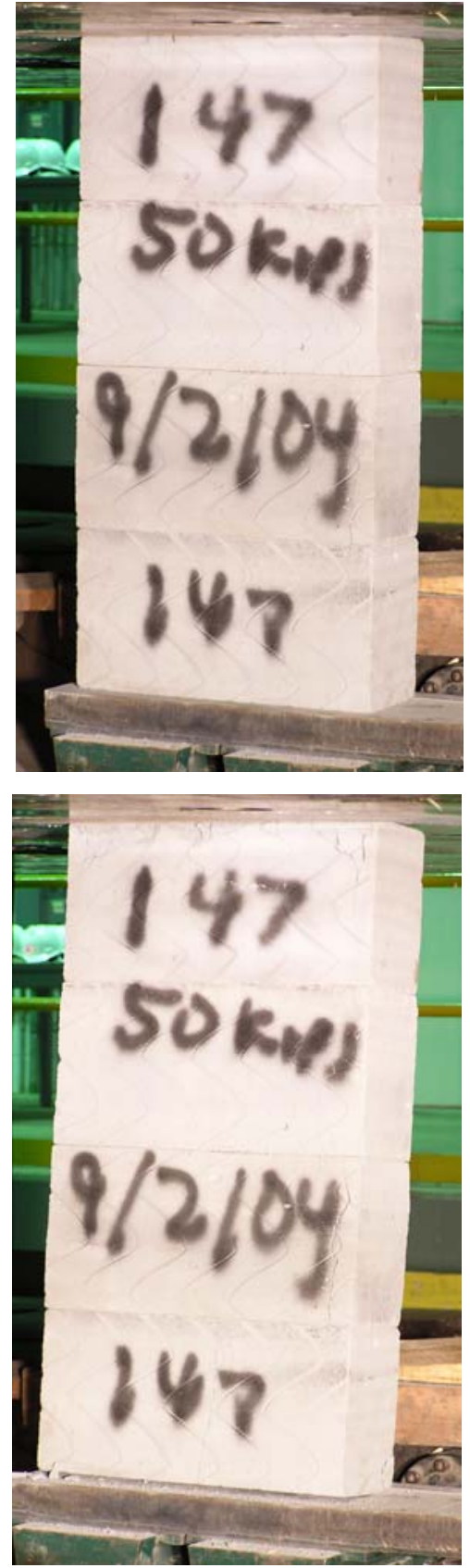

Figure 5-8a. Test of a half-wall made from lightweight block (546-psi compressive strength).
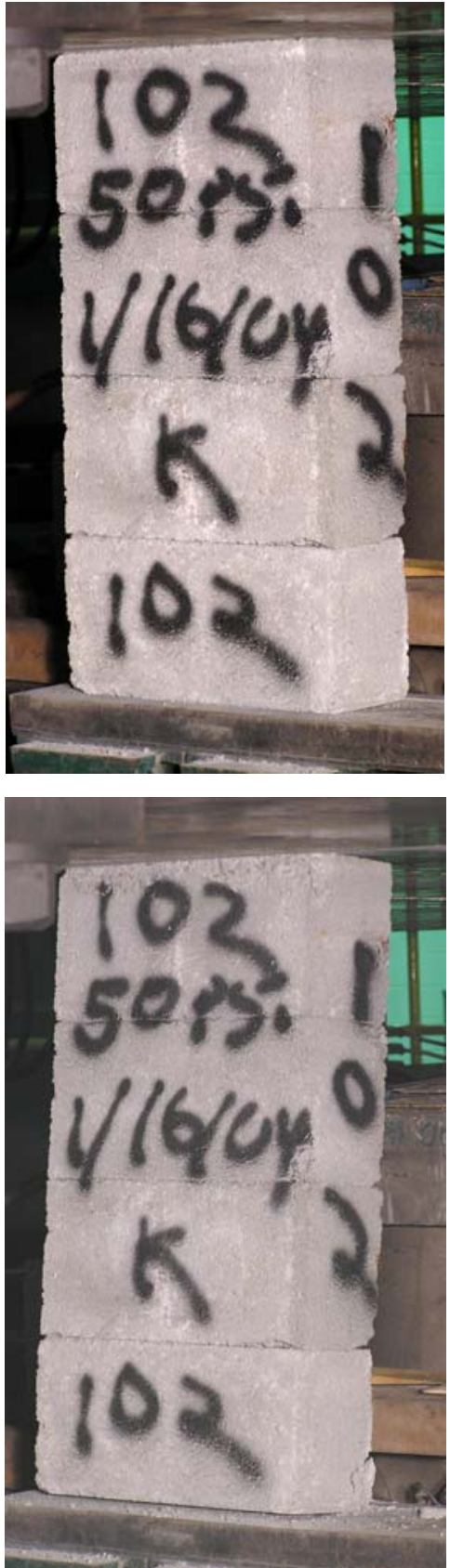

Figure 5-8b. Test of a half-wall made from conventional concrete block (1,330-psi compressive strength). 


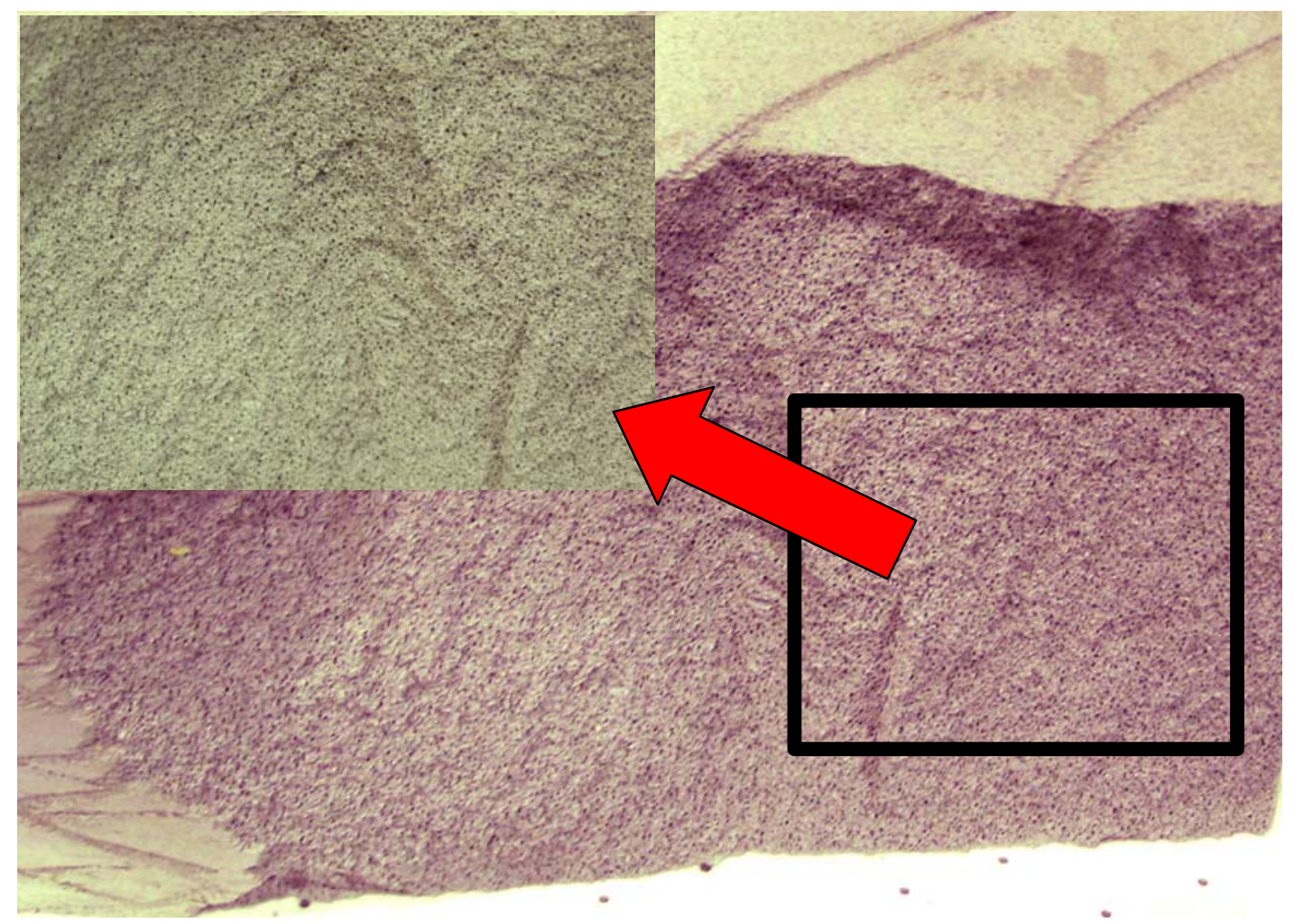

Figure 5-9. Close up view of Kingsway, autoclaved, concrete block shows air pockets in the block structure.

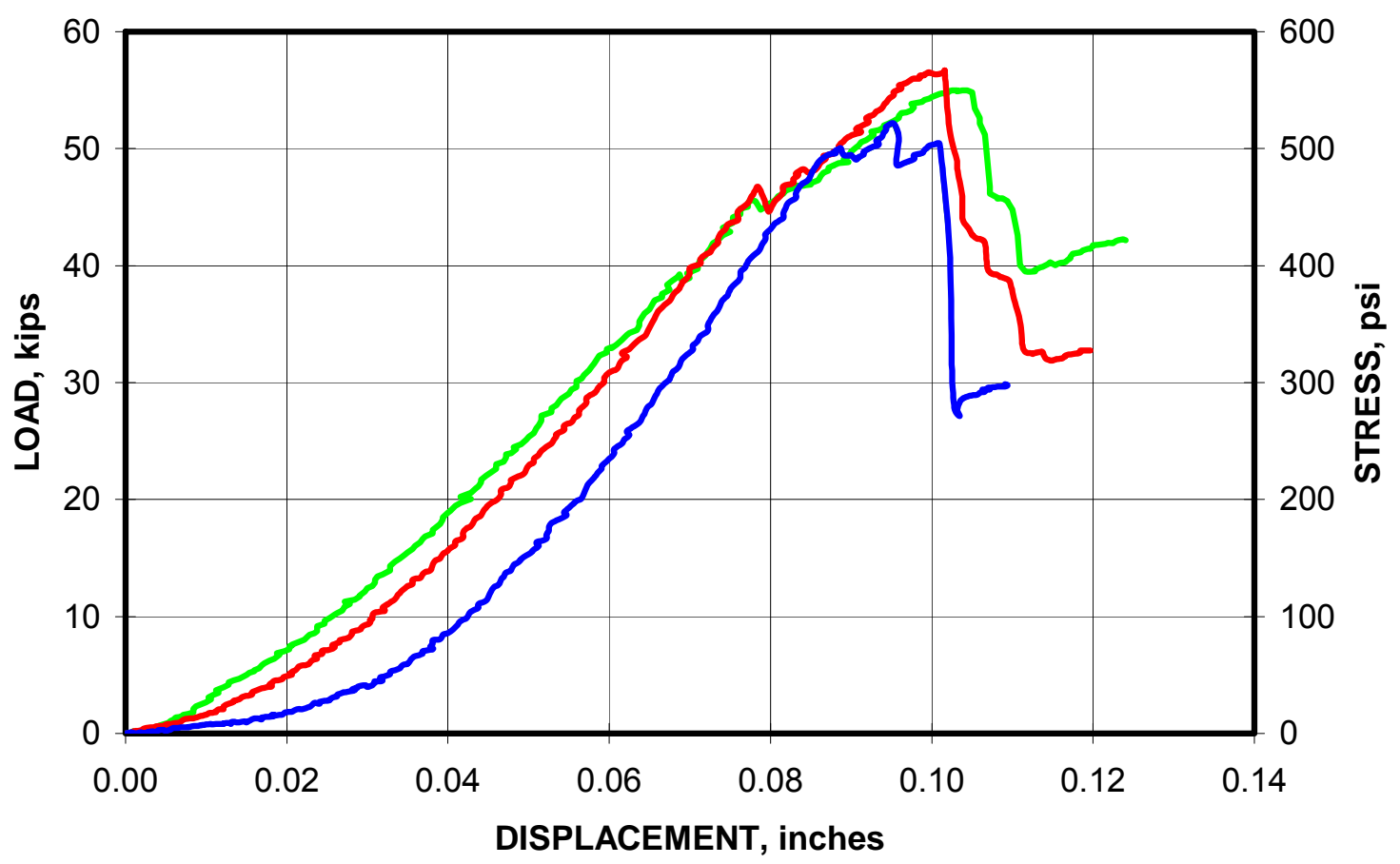

Figure 5-10. Compressive strength test data for Kingsway block. 


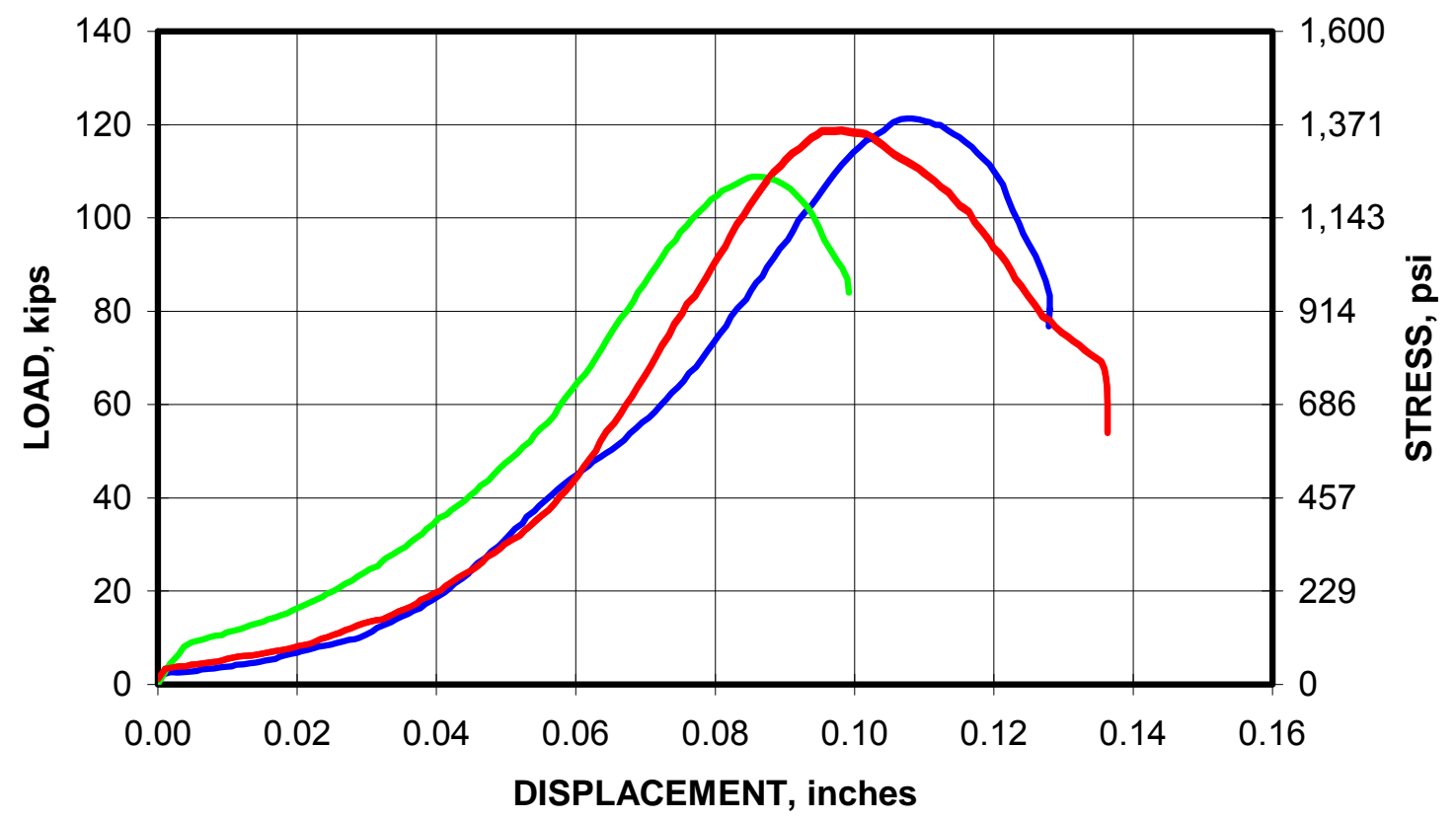

Figure 5-11. Compressive strength test data for conventional concrete block.

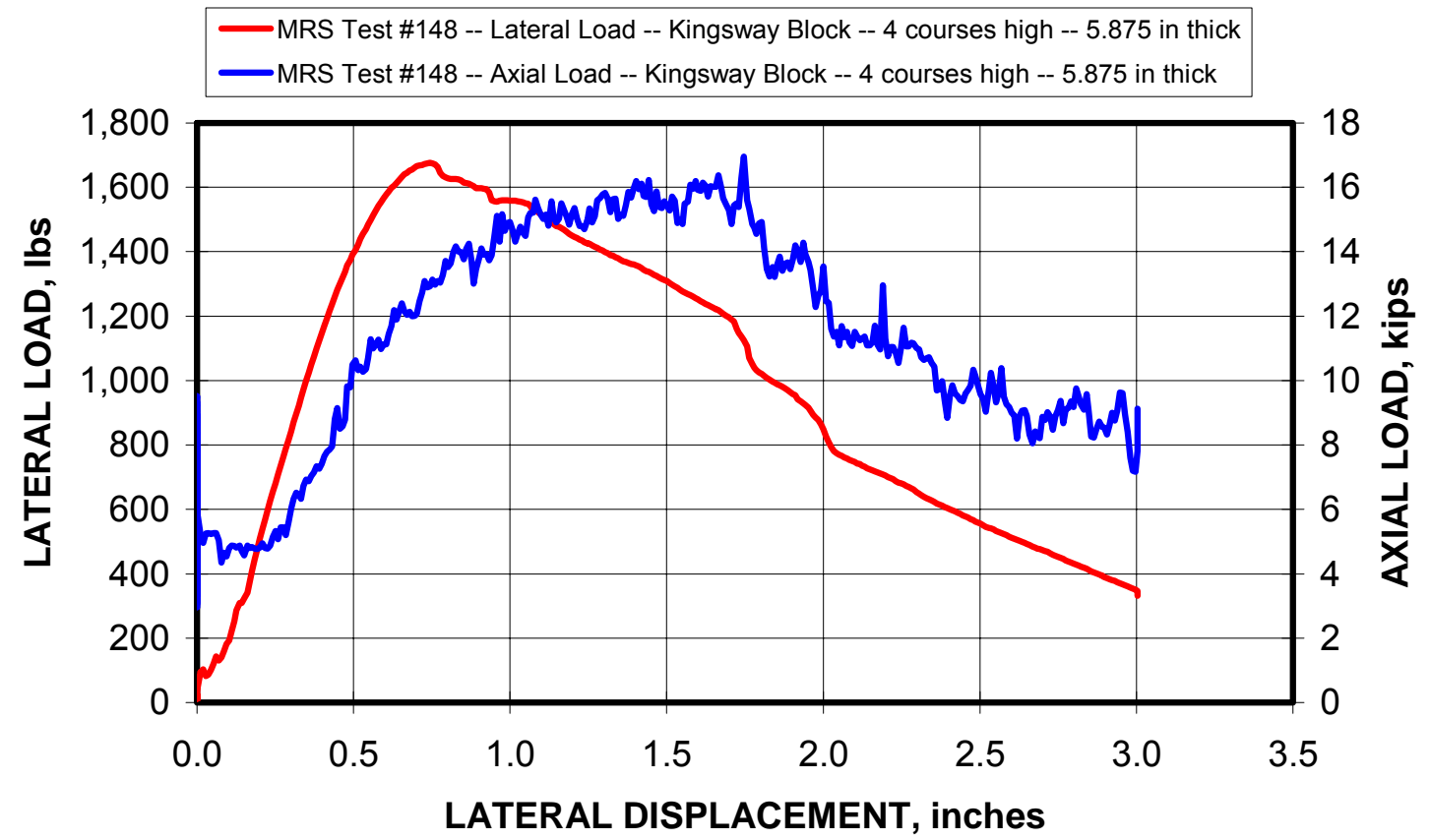

Figure 5-12. Half-wall rigid-arching tests conducted in the Mine Roof Simulator on the Kingsway lightweight block. 
Figure 5-12 documents the half-wall rigid arching test results for the lightweight (Kingsway) block. The graph plots the applied lateral load provided by the load frame to produce the controlled lateral displacement of the wall. A nominal axial preload of approximately $50 \mathrm{psi}$ was applied to the ends of the wall by the load frame prior to the initiation of the lateral displacement of the base of the wall. The preload, which typically would be provided by wedging the wall in place in an actual stopping construction, ensures a tight fit and a fully restrained end condition. The graph shows that the lateral load increases with increasing lateral displacement up to the peak load, which in this test was approximately $1,675 \mathrm{lbs}$ occurring at 0.74 inches of lateral displacement. It is also seen from figure 5-12 that the axial (vertical) reaction load measured by the load frame since the vertical opening of the platens is held constant, also increases as the lateral loading increases. This is consistent with arching theory.

The measured vertical force is equivalent to the arching force or thrust $(\mathrm{P})$ as defined in equation 4.3. During the initial lateral displacement of the wall, the arching thrust or vertical loading decreases slightly and then remains relatively constant for about 0.25 inches of lateral displacement in this particular example. The arching thrust is a function of the elastic properties of the concrete block and the contact area developed as the wall rotates. As the wall initially rotates, the very edge of the block is loaded in compression, and the contact area is changing rather quickly as localized failures occur at the block edge, since the mold in which the block is formed results in a somewhat ragged and uneven edge. Once the contact moves beyond the immediate edge of the block, the contact area becomes more controlled and the arching thrust, or axial load as indicated in the graph, increases. There may also be some load frame control interactions as the lateral displacement is first applied that can cause a temporary drop in the apparent axial load. The kinematics of the wall suggests that the contact area will decrease with increasing lateral deflection. This essentially causes an increase in the stress acting on the crushing zone until the compressive strength of the material is reached. In reality, although the wall is loaded in compression, the actual failure of the material is more likely to be governed by the shear strength of the material. Once the compressive (or shear strength) of the material is exceeded, the axial load will decrease. 
The peak axial load in this particular example occurred at a larger lateral displacement than where the peak lateral load was observed. Although this may seem contradictory at first glance, the answer lies in the formation and continued development of the crush zone produced by the arching thrust. Reference is made back to equation 4.1, which shows that the transverse load moment is resisted by the thrust moment. As lateral displacement is applied, the width of the arch (r) as shown in figure 4.2 decreases. However, the arching thrust is increasing quickly during this loading phase, allowing the transverse load to increase as well. Once the transverse load peaks, the axial load continues to increase since the strength of the block has not yet been exceeded. The peak transverse load is controlled by the force couple balance between the thrust moment and the transverse load moment. The continued growth and more accurately movement of the crush zone away from the edge of the block as the lateral displacement increases determines when the peak transverse load will occur. The decrease in transverse load following the peak transverse loading does not prevent the axial load from continuing to increase. Again, all that is required is that the moments balance, and during this phase where than transverse loading is decreasing while the axial load continues to increase slowly, the arch width (r) can be decreasing more than the axial load is increasing, and this can allow for the condition observed in the test. The distribution of stresses in the crush zone can be quite complex and is considered beyond the scope of this thesis. The objective of this thesis is only to determine if arching provides a more accurate representation of the true transverse load capacity of stoppings and to develop a test procedure to simulate this loading condition.

The applied lateral load can then be normalized to the area of the wall to provide a transverse load capacity measured in pounds per square foot or psf. For the example shown in figure 5-12, the four-course, single-block column was 17.25 inches wide by 33.50 inches high providing an area of $577.88 \mathrm{in}^{2}$ or $4.01 \mathrm{ft}^{2}$. Dividing the lateral load from the test $(1,675 \mathrm{lbs})$ by this area provides a transverse load capacity of $417 \mathrm{psf}$. This is an order of magnitude higher than the $39 \mathrm{psf}$ required by the current CFR criteria based on a freestanding wall analysis.

Figure 5-13 shows the test results from the second example using conventional concrete block, or conventional masonry units (CMU) as they are sometimes called. This 
block has a compressive strength of 1,330 psi (figure 5-11) or about 2.5 times that of the autoclaved block tested in the first example. As seen in figure 5-13, the peak lateral load acting on this wall was $3,855 \mathrm{lbs}$ occurring at a lateral displacement of $1.02 \mathrm{in}$. This equates to a transverse load of 1,067 psf or 2.6 times that of the autoclaved block used in the previous test. It is noted that the difference in lateral load capacity between the lightweight and the conventional block is consistent with the difference in material strength. This provides additional validation for the application of arching theory to stopping wall behavior.

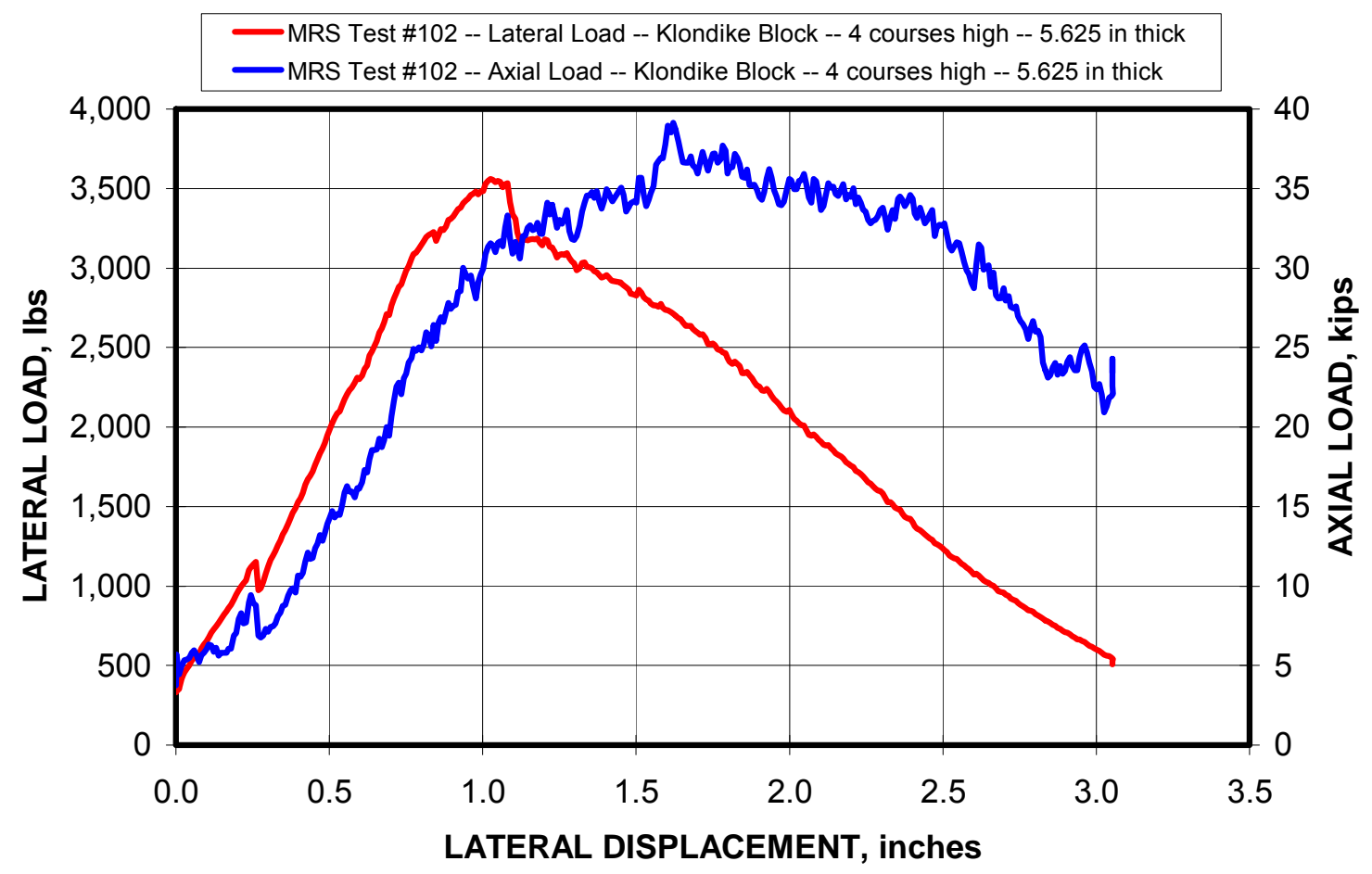

Figure 5-13. Half-wall rigid-arching tests conducted in the Mine Roof Simulator on conventional concrete block.

\subsection{COMPARISON TO RIGID ARCHING THEORETICAL DESIGN}

In order to evaluate these laboratory results compared to the rigid arching design equation formulated in equation 4.5, a few other combinations of wall thickness and height were tested using the MRS biaxial testing protocol for the two block types evaluated in the two previous examples. A higher strength solid concrete block was also tested. These wall specifications are summarized in tables 5-1 and 5-2 for the Kingsway 
and the Klondike block constructions, respectively. The parameters controlled in this study were the height of the wall (or half-wall height in the test) and the block orientation (narrow or wide side block-on-block contact). The individual test results for all halfwall tests conducted in the MRS are documented in Appendix C.

Table 5-1. Specifications for MRS biaxial testing on Kingsway block walls.

\begin{tabular}{|l|c|c|c|c|c|}
\hline $\begin{array}{c}\text { Block type } \\
\text { compressive } \\
\text { strength, psi) }\end{array}$ & $\begin{array}{c}\text { Thickness, } \\
\text { in }\end{array}$ & $\begin{array}{c}\text { Block } \\
\text { height, in }\end{array}$ & $\begin{array}{c}\text { Half-wall } \\
\text { height, in }\end{array}$ & $\begin{array}{c}f_{\mathrm{c}} \times(\mathrm{t} / \mathrm{L})^{2}, \\
\mathrm{psi}\end{array}$ & $\begin{array}{c}\text { Transverse } \\
\text { load, psf }\end{array}$ \\
\hline Kingsway (546) & 5.875 & 8.375 & 41.875 & 2.69 & 194 \\
\hline Kingsway (546) & 5.875 & 8.375 & 41.875 & 2.69 & 231 \\
\hline Kingsway (546) & 5.875 & 8.375 & 58.625 & 1.37 & 87 \\
\hline Kingsway (546) & 5.875 & 8.375 & 58.625 & 1.37 & 69 \\
\hline Kingsway (546) & 5.875 & 8.375 & 41.875 & 2.69 & 224 \\
\hline Kingsway (546) & 5.875 & 8.375 & 41.875 & 2.69 & 203 \\
\hline Kingsway (546) & 5.875 & 8.375 & 50.250 & 1.87 & 113 \\
\hline Kingsway (546) & 5.875 & 8.375 & 33.500 & 4.20 & 379 \\
\hline Kingsway (546) & 5.875 & 8.375 & 33.500 & 4.20 & 417 \\
\hline
\end{tabular}

Table 5-2. Specifications for MRS biaxial testing on Klondike block walls.

\begin{tabular}{|l|c|c|c|c|c|}
\hline $\begin{array}{c}\text { Block type } \\
\text { compressive } \\
\text { strength, psi) }\end{array}$ & $\begin{array}{c}\text { Thickness, } \\
\text { in }\end{array}$ & $\begin{array}{c}\text { Block } \\
\text { height, in }\end{array}$ & $\begin{array}{c}\text { Half-wall } \\
\text { height, in }\end{array}$ & $\begin{array}{c}f_{\mathrm{c}} \mathrm{x}(\mathrm{t} / \mathrm{L})^{2}, \\
\mathrm{psi}\end{array}$ & $\begin{array}{c}\text { Transverse } \\
\text { load, psf }\end{array}$ \\
\hline Klondike (1330) & 5.625 & 7.50 & 30.00 & 11.69 & 1,157 \\
\hline Klondike (1330) & 5.625 & 7.50 & 30.00 & 11.69 & 1,068 \\
\hline Klondike (1330) & 5.625 & 7.50 & 30.00 & 11.69 & 843 \\
\hline Klondike (1330) & 5.625 & 7.50 & 45.00 & 5.03 & 220 \\
\hline Klondike (1330) & 5.625 & 7.50 & 45.00 & 5.03 & 203 \\
\hline Klondike (1330) & 5.625 & 7.50 & 45.00 & 5.03 & 255 \\
\hline Klondike (1330) & 5.625 & 7.50 & 60.00 & 2.92 & 48 \\
\hline Klondike (1330) & 5.625 & 7.50 & 60.00 & 2.92 & 51 \\
\hline Klondike (1330) & 7.500 & 5.75 & 46.00 & 8.84 & 651 \\
\hline Klondike (1330) & 7.500 & 5.75 & 46.00 & 8.84 & 617 \\
\hline Klondike (1330) & 7.500 & 5.75 & 46.00 & 8.84 & 576 \\
\hline Klondike (1727) & 5.625 & 7.50 & 30.00 & 15.18 & 1,467 \\
\hline Klondike (1727) & 5.625 & 7.50 & 30.00 & 15.18 & 1,375 \\
\hline Klondike (1727) & 5.625 & 7.50 & 30.00 & 15.18 & 1,425 \\
\hline
\end{tabular}


Figures 5-14 and 5-15 compare the transverse load determined from the MRS halfwall rigid-arch tests to the theoretical prediction using equation 4.5. In these figures, the transverse load is plotted against the term $f_{\mathrm{c}} \times(\mathrm{t} / \mathrm{L})^{2}$ (compressive strength of the material times the square of the ratio of the wall thickness to the wall height). As seen in these figures, the transverse load is linearly related to the $f_{\mathrm{c}} \times(\mathrm{t} / \mathrm{L})^{2}$ term, in accordance with moment equilibrium for the theoretical assessment of rigid arching statics. The regression equations show an $\mathrm{R}^{2}$ of 0.98 for the Kingsway block and for the Klondike block as well, indicating that the parameters in this term largely control the transverse loading capability of the stopping wall. The slope of the theoretical curve parallels that of the linear regression line for the MRS half-wall tests in each case, but the theoretical predictions of transverse load are consistently higher than the MRS test results. The negative intercept for the MRS regression lines indicate that the trend lines are not valid as the term $f_{\mathrm{c}} \times(\mathrm{t} / \mathrm{L})^{2}$ falls below a certain value that produces a negative transverse load, since this is physically not possible. This suggests that the transverse load is nonlinearly related to the $f_{\mathrm{c}} \times(\mathrm{t} / \mathrm{L})^{2}$ term as it approaches a zero value, or other factors are controlling in these conditions. However, the range of data presented in the regression curve is realistic for stopping constructions, and no further assessment of this specialized condition dictated by high wall heights, and/or thin walls, and low material strengths.

The arching theory represents an extreme idealization of the behavior of endrestrained conditions, both with respect to the geometry of the lateral wall movement and the stress-strain properties of the material. Specifically, the assumptions made in the theoretical design equation illustrated in figures 5-14 and 5-15 include: (1) a "crush zone" thickness equal to an arbitrary one-tenth the thickness of the wall, (2) a uniform loading distribution across this area, and (3) identical behavior at the ends of the wall and at the mid-span joint. Modifications of these various assumptions can alter the predicted transverse loading to varying degrees, but the different offset between the two block types indicate that a single correction factor is not feasible. Closer examination of the regression equations compared to the theoretical predictions show the offset in transverse load is greater for the higher strength Klondike block compared to the lower strength Kingsway block. In view of the stochastic nature of the physical properties of concrete materials, since these two blocks are significantly different materials, the Kingsway block 
is an autoclaved concrete material full of air pockets (figure 5-9) while the Klondike block is a conventional cement aggregate material, their failure behavior is likely to be quite different as well. In addition to the assumptions listed above, shearing failure is not directly represented in this idealized theory, although the failure mechanism of the concrete most likely involves shearing action. Hence, it is unlikely that the idealized theoretical assessment of rigid-arching would exactly predict physical testing, or in this case, the MRS half-wall test results. However, the regression analysis of the test data shows that the compressive strength, wall thickness, and height of wall, as expressed in the $f_{\mathrm{c}} \times(\mathrm{t} / \mathrm{L})^{2}$ term, account for $98 \%$ of the variation in the ultimate transverse load in rigid-arch loading of stopping walls.

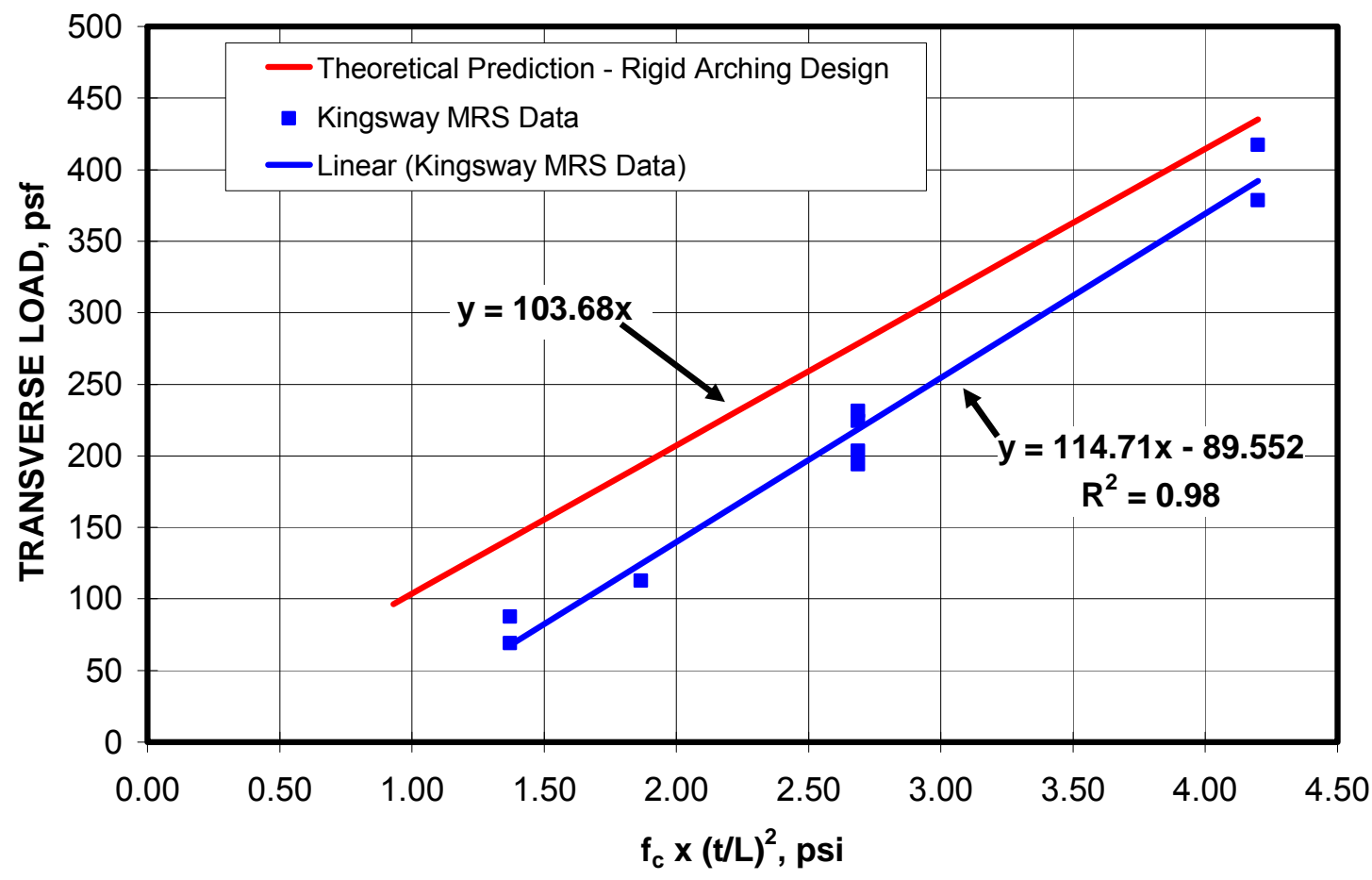

Figure 5-14. Comparison of MRS half-wall rigid-arch testing to the theoretical prediction of transverse loading for Kingsway block stoppings. 


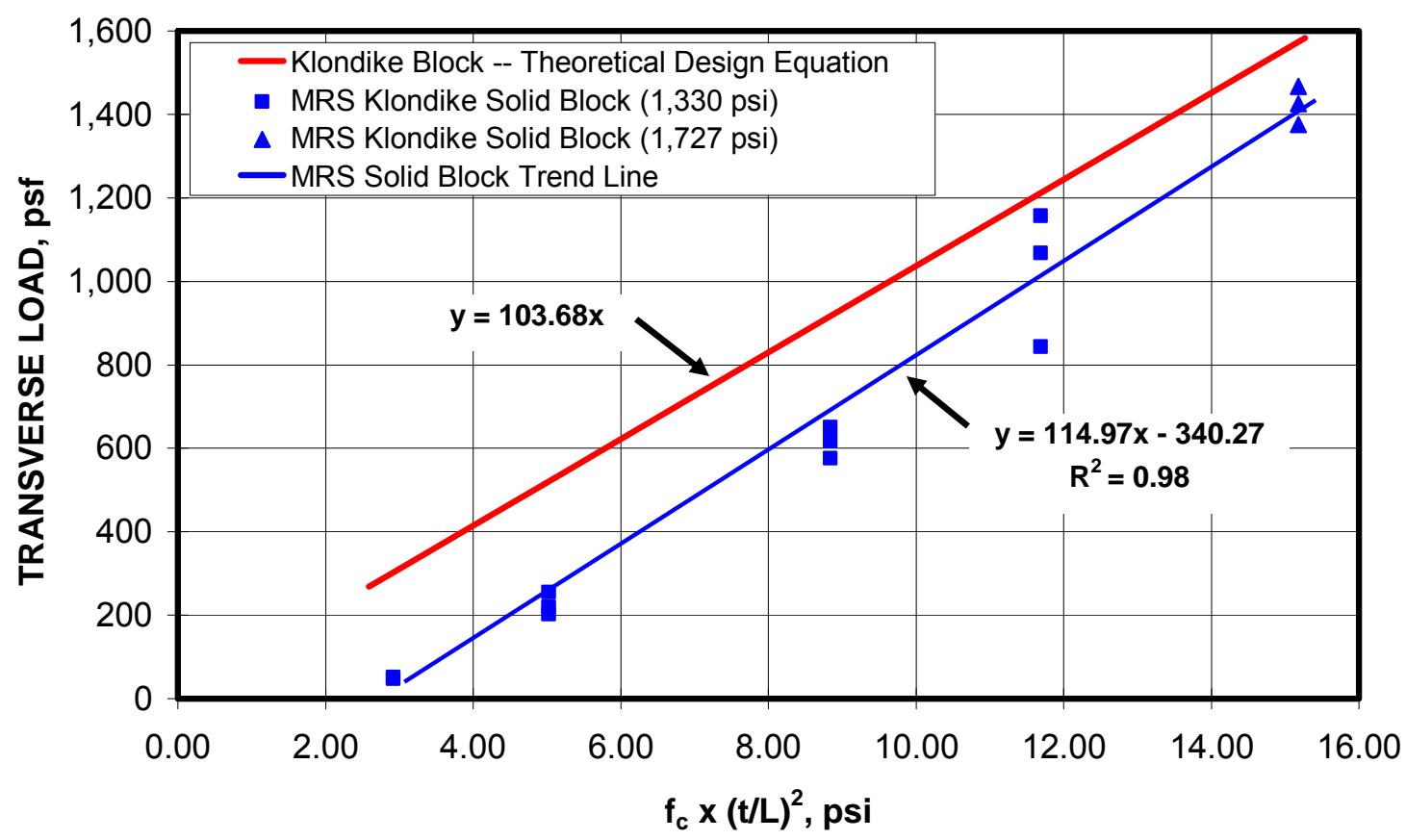

Figure 5-15. Comparison or MRS half-wall rigid-arch testing to the theoretical predictions of transverse loading for Klondike block stoppings. 


\section{CHAPTER 6. FULL-SCALE LOAD VERIFICATION TESTING}

In order to confirm that arching was the proper loading mechanism controlling the transverse load capacity of mine ventilation stoppings and to verify the MRS half-wall rigid-arching testing protocol, a few full-scale tests of stopping walls were also conducted. These tests were conducted in the NIOSH Experimental Coal Mine at the Pittsburgh Research Laboratory. Test data was also analyzed from explosion testing of full-scale stoppings at the NIOSH Lake Lynn Laboratory.

\subsection{NIOSH PRL EXPERIMENTAL COAL MINE TESTS}

The Experimental Coal Mine is an abandoned coal mine that has been used as an underground laboratory for conducting various research experiments by NIOSH researchers. The mine has been part of the Bureau of Mines since 1910 and is now owned by NIOSH. It is located on site at the Pittsburgh Research Laboratory near Bruceton, PA.

\subsubsection{Air Pressure Chamber}

An air pressure chamber was constructed in one of the crosscuts in the mine to provide a facility for static loading of mine ventilation stoppings. The crosscut measures approximately $16 \mathrm{ft}$ in width and about 80 inches in height. Figure 6-1 shows a diagram of the chamber. A barrier wall was constructed from mortared high strength solid concrete blocks. The barrier is 16 inches thick. An access door, air intake port, and data acquisition lead wire ports were installed during construction of the barrier. A stopping wall is then constructed approximately three ft from the barrier wall. A concrete pad was formed on the floor of the crosscut to provide a flat foundation for constructing the stopping wall. The pillar ribs were also squared up, again to facilitate the stopping wall construction in order to minimize air leakage that might occur along this interface.

The test wall is constructed in a normal dry-stacked fashion. The top of the wall is tightened with wood wedges and the gaps are filled with mortar and sealant to prevent air leakage. The inside or high-pressure side of the stopping is also coated with sealant to prevent air leakage through the block joints. The sealant placed on this side of the wall 
does not significantly contribute structurally to the transverse load capacity of the wall. The sealant would have to be placed on the opposite or low-pressure side of the wall to contribute. For this reason, no sealant was applied to the low-pressure side of the wall.

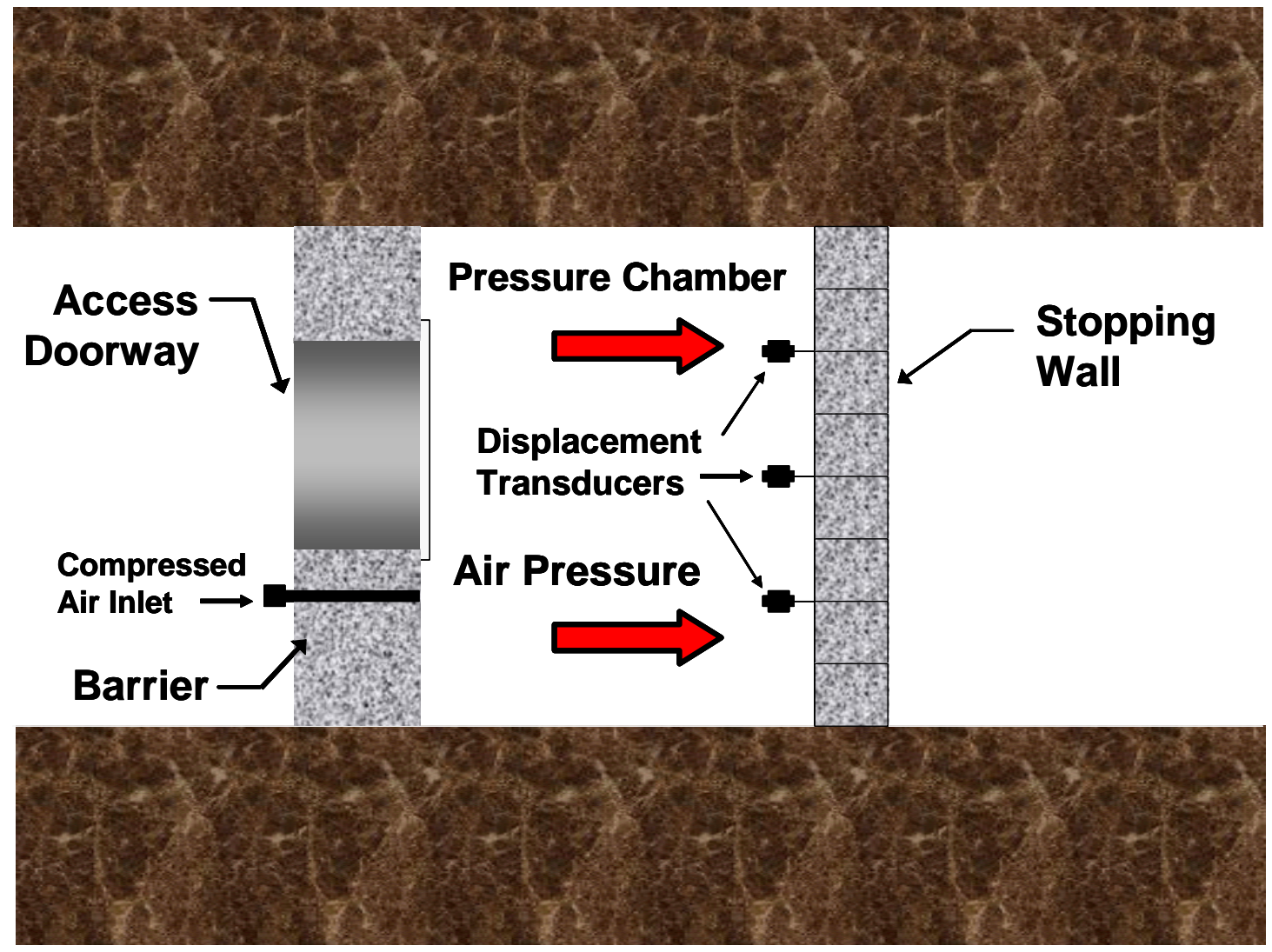

Figure 6-1. Diagram of the pressure chamber in the NIOSH Experimental Mine used for conducting full-scale transverse loading tests.

Pressurized air is injected into the chamber between the barrier and the test wall through the air intake port. The air pressure is provided by an air compressor capable of supplying $560 \mathrm{cu} \mathrm{ft}$ per minute of air at a pressure of 120 psi. A pressure transducer attached to a secondary inlet port into the pressure chamber measures the pressure in the chamber. In this case, a leakage rate evaluation is made only to determine if the air compressor will have sufficient capacity to develop the necessary air flow and pressure to fail the wall, although, this is the same procedure that would be followed if a more detailed assessment of the leakage rate was made. Initial checks of leakage are made at 5 inches of water gage (approximately $0.18 \mathrm{psi}$ ). The air pressure is increased gradually 
in increments by adjusting a control valve on the pressure line. This process continues until the air pressure in the chamber blows out the wall. Three displacement transducers are utilized to measure the lateral displacement at the mid and quarter point heights of the wall as the pressure is applied (figure 6-1).

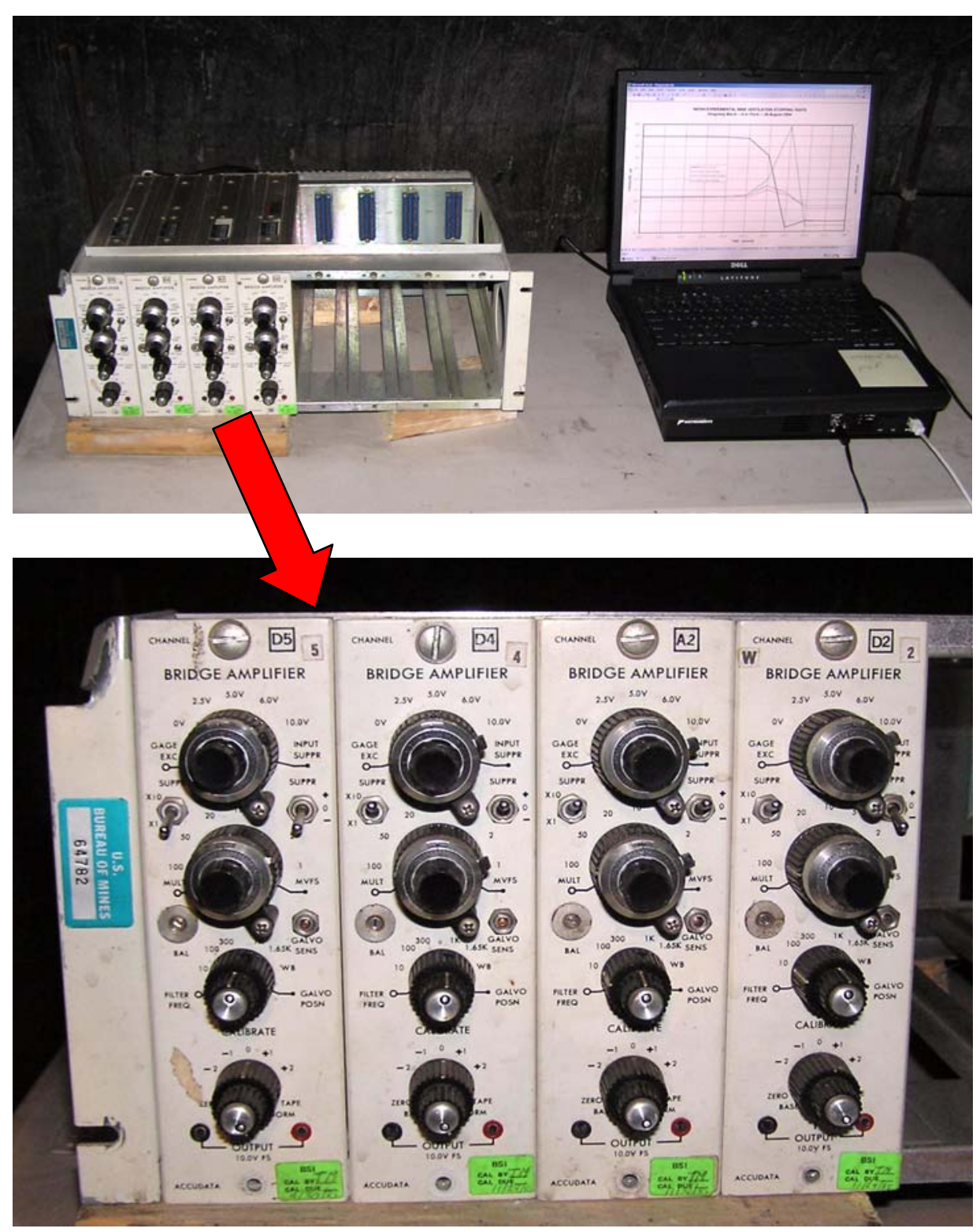

Figure 6-2. Data acquisition system to measure air pressure and wall displacement for the full-scale stopping wall testing in the NIOSH Experimental Coal Mine.

The conversion of physical measurement parameters to output voltage is accomplished using Honeywell Accudata Model 218 Bridge Amplifiers. The output 
from each amplified sensor channel is converted to a digital value utilizing a National Instruments DAQPad-6020E. The DAQPad-6020E is controlled and data is transferred to a Dell Latitude laptop computer running National Instruments VI Logger software under the Microsoft Windows 2000 operating system. Test data files are then converted to Microsoft Excel Workbook format for further processing and analysis. A photo of the data acquisition system is shown in figure 6-2 and a complete description of the data acquisition system is provided in Appendix D.

\subsubsection{Test Results and Comparisons to Half-Wall MRS Tests}

Two full-scale wall tests were conducted in the NIOSH Experimental Coal Mine. The first test utilized the lightweight autoclaved blocks that were used in the first example presented for the MRS rigid-arching tests in Section 5.3 of this report. The second test was a wall constructed from the conventional solid concrete aggregate block that was utilized in the second MRS rigid-arching test. This was done so that a direct comparison to these tests could be made.

The results of the lightweight autoclaved block test in comparison to the full-scale mine test are shown in figure 6-3. As seen in the figure, the single-column, half-wall rigid-arching test conducted in the MRS closely predicted the full-scale wall behavior in the mine. The peak transverse loading was $417 \mathrm{psf}$ for the MRS test and $400 \mathrm{psf}$ for the full-scale mine test. The stiffness of the walls relative to transverse load and deflection at the mid-span of the wall are also similar, the full-scale stopping response in the mine was slightly stiffer than the MRS half-wall response. Transverse load development in the MRS wall was delayed compared to the full-scale mine test, but this is likely due to the initial conditions previously discussed with regard to the initial rotation or deflection of the wall in the MRS test. The result of this action is that the peak lateral load in the MRS test occurred at about 0.4 more inches of lateral displacement of the wall compared to the full-scale mine test. Figure 6-4 compares the full-scale mine test with all the MRS halfwall tests identified in table 5-1. As seen in this figure, the full-scale mine test is very close to the regression trend line developed from the suite of MRS tests, and in fact, it falls directly between the two MRS tests that simulate the full-scale mine test. 


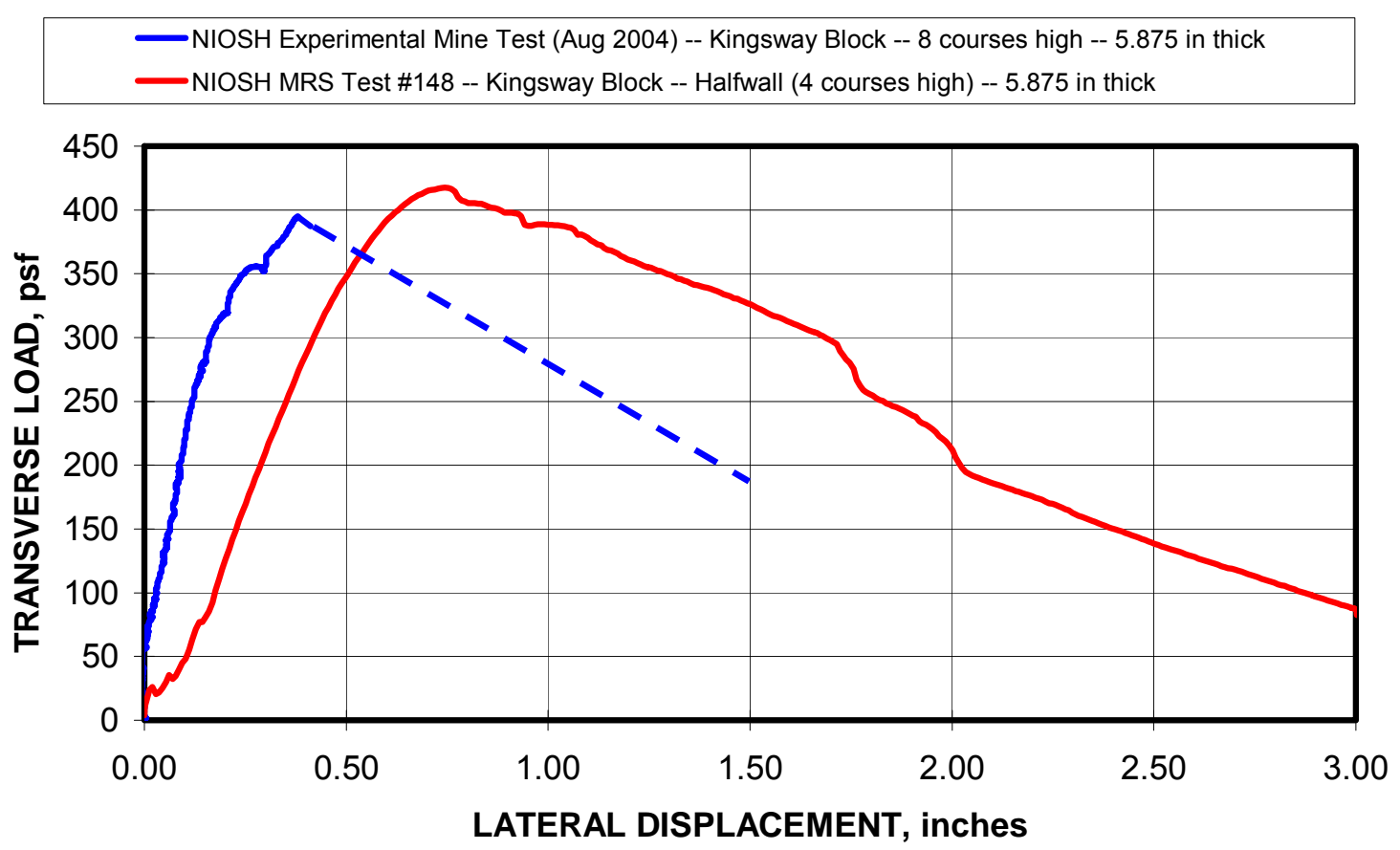

Figure 6-3. Comparison of half-wall rigid-arch test in the MRS to the full-scale stopping wall test in the NIOSH Experimental Coal Mine for the Kingsway block.

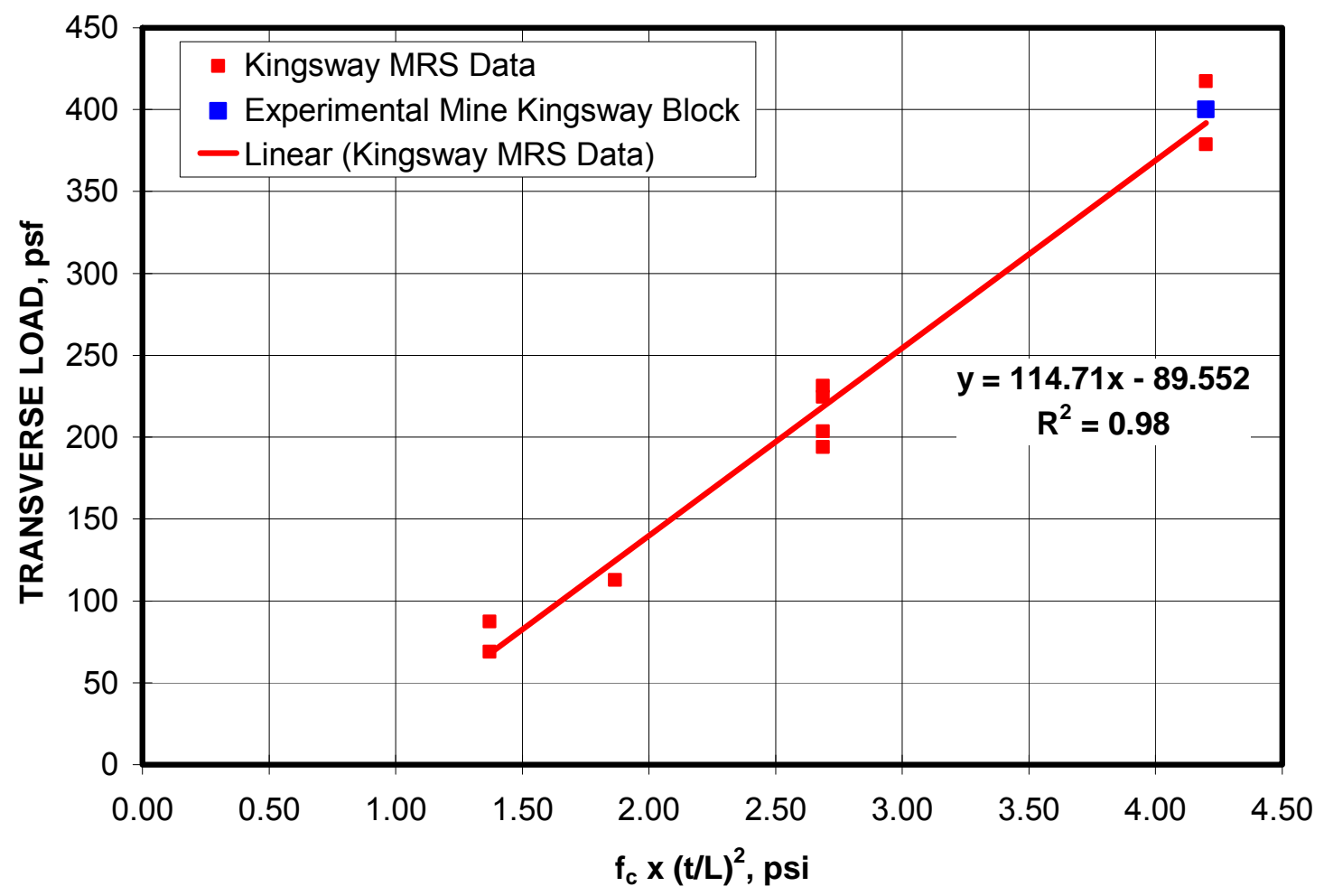

Figure 6-4 Comparison of full-scale mine test of Kingsway block with the MRS half-wall regression trend line and suite of MRS tests. 
Photos of the remains of the stopping after the full-scale mine test are shown in figures 6-5 and 6-6. It is seen in these figures that the bottom and top course of block stayed in place after the wall was blown out. This is most likely because the bottom course was grouted in place to provide a level and secure foundation to build the wall. Likewise, cement grout was squeezed into the voids on top of the wall between and around the wooden wedges to stop air leaks, and this secured the top block to the shotcreted roof in the Experimental Coal Mine. As such, the functional wall height relative to the arching length was the eight courses of block between the top and bottom layer as denoted in figure 6-6.

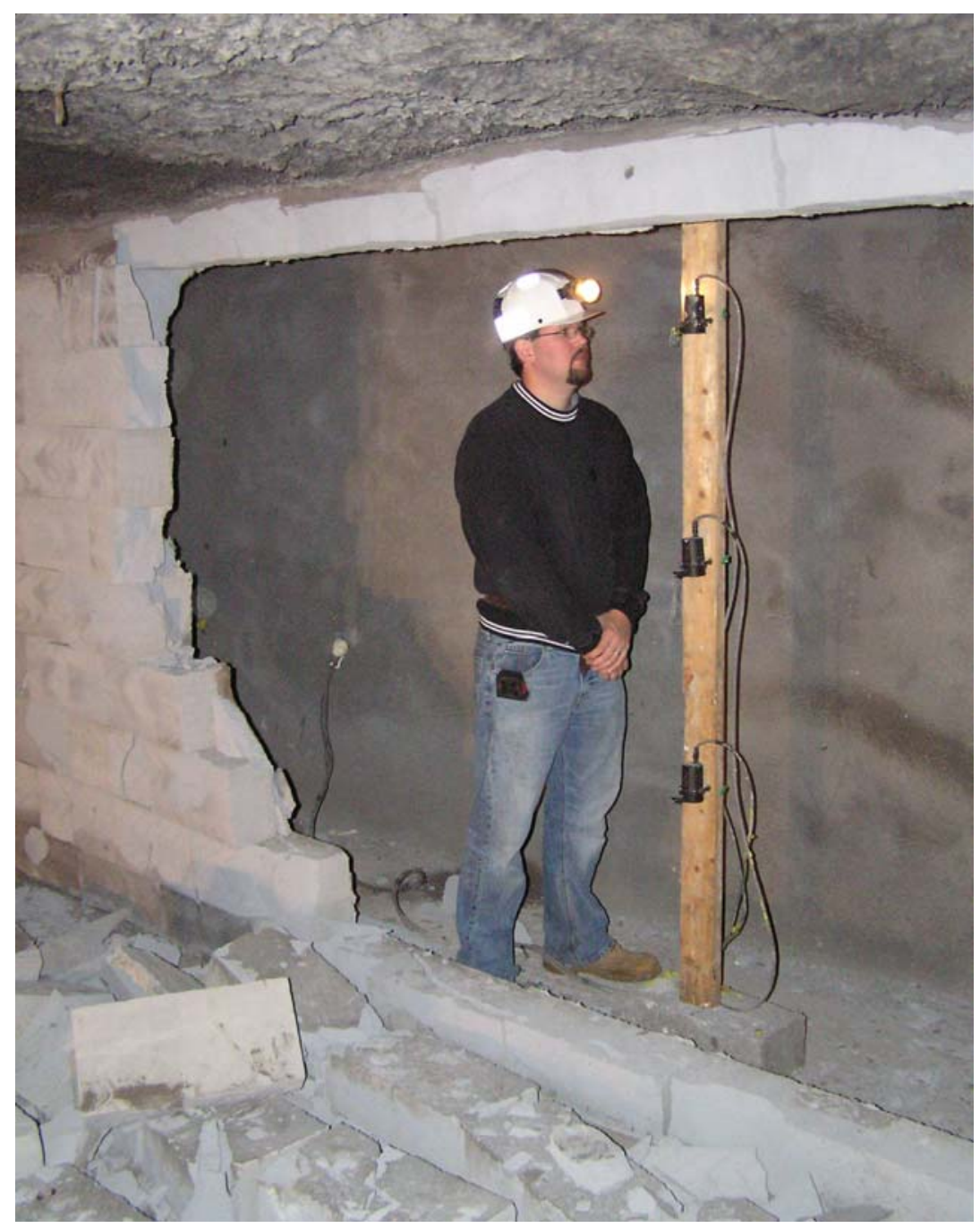

Figure 6-5. Photo after the wall was destroyed from the transverse loading. Researcher is standing next to the displacement transducers used to measure the wall deflection. 


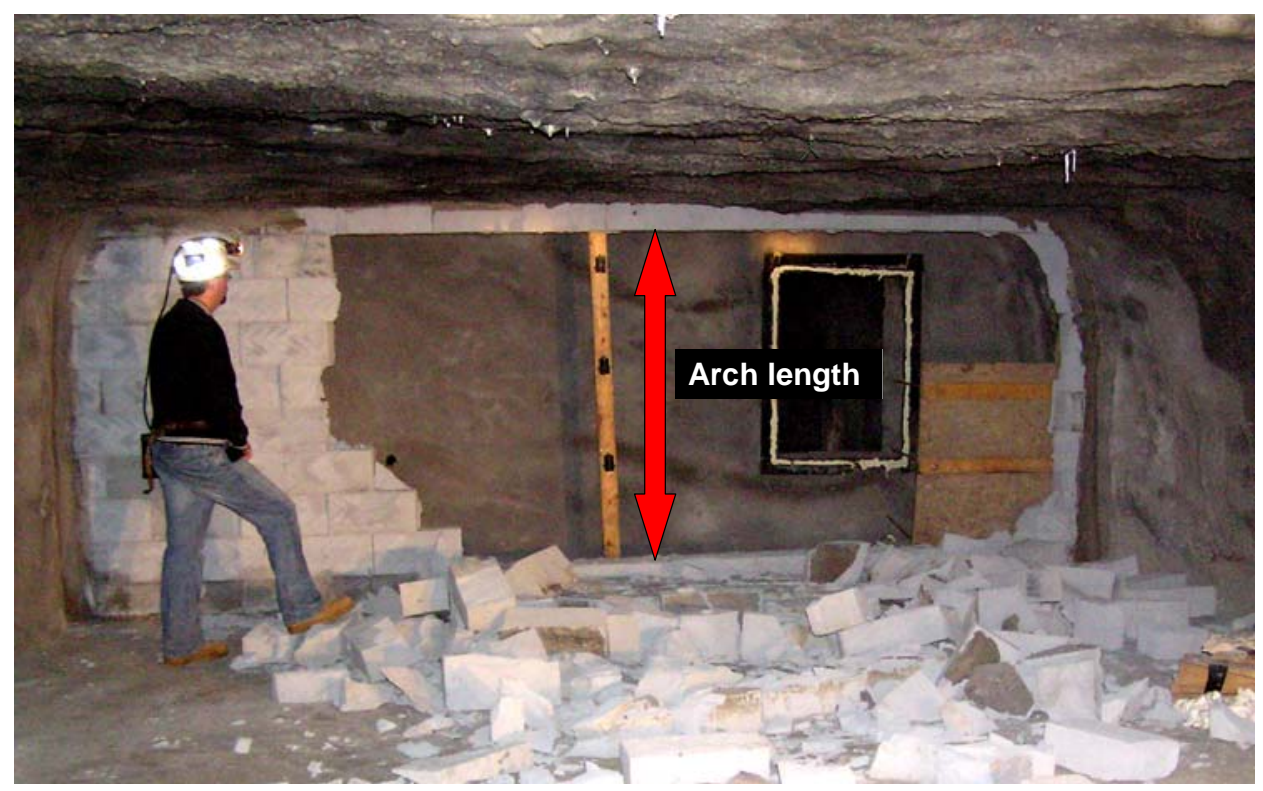

Figure 6-6. Arch height shown to occur between top and bottom layer of block that were grouted (cemented) in place in this particular test.

The comparisons of the MRS half-wall rigid-arching test to the full-scale mine test for the walls constructed from the conventional Portland cement, sand, and aggregate block manufactured by Klondike are shown in figure 6-7. As seen in the figure, the single-column, half-wall, rigid-arching test conducted in the MRS reasonably predicted the full-scale wall behavior in the mine. The peak transverse loading was 1,067 psf for the MRS test and 975 psf for the full-scale mine test. As with the Kingsway block reported in the previous analysis, the full-scale mine wall made from the Klondike block exhibited an even stiffer response than that observed in the MRS laboratory test. Again, this is believed to be due partially to the nature of the MRS tests in terms of the initial conditions. It may also be due, in part, to the fact that only one point of displacement measurement is made on the full-scale wall at the mid-span location, and this may not be the point of maximum deflection in the wall. In addition, the restraint provided in the lateral direction by the coal pillars may have some influence on the lateral deflection of the wall. As seen in figure 6-7, the full-scale stopping wall appeared to reduce its stiffness once the loading reached about 900 psf at 0.35 inches of lateral displacement, and then increased its stiffness at about 0.65 inches of lateral displacement where it is suspected that the wall slipped some. The airflow rate was not controlled in this 
experiment, and this response may also be due to increased leakage rates within the pressure chamber at the higher pressures where the air supply was approaching maximum airflow as the wall deflected. Nonetheless, the peak transverse loading and the overall response of the full-scale mine test was similar to that observed in the single-column, half-wall test conducted in the MRS. A comparison of the full-scale mine test to the regression trend line developed from the suite of MRS tests documented in table 5-2 is shown in figure 6-8. As seen in this figure, the full-scale test was very close to the MRS trend line.

Photos of the remains of the full-scale stopping test with the conventional Klondike block are shown in figures 6-9 through 6-11. Figure 6-9 shows the arching length occurred between the top and bottom course of block, just as was observed with the Kingsway block in the previous test. Figure 6-10 shows the bottom course of block which remained in place after the test. As seen is this photo, the outer region of the block shows shear failure damage to the contact area where the hinging occurred. Figure 6-11 shows a close up view of the base of the wall illustrating the rotation of the wall and formation of the bottom hinge of the arch.

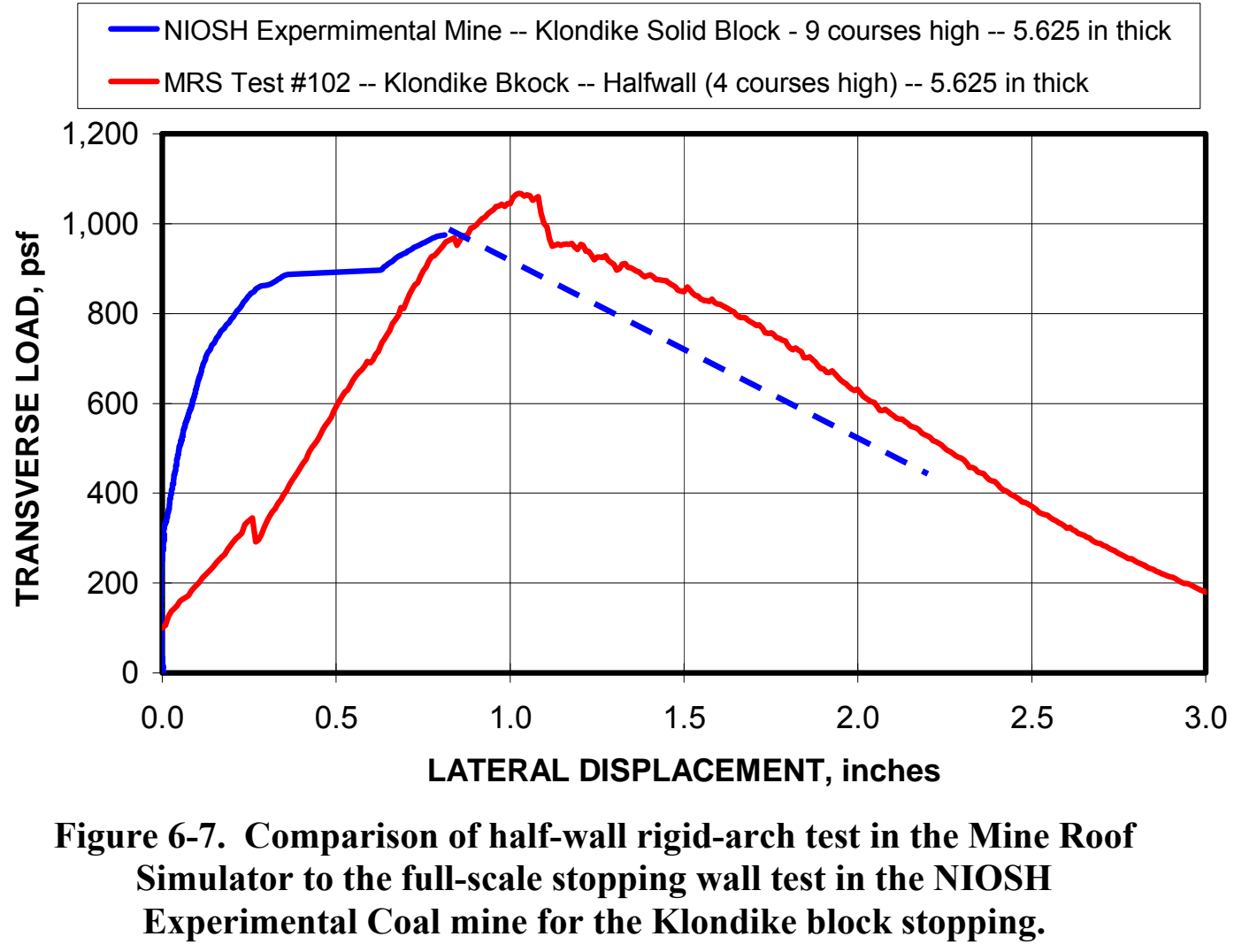




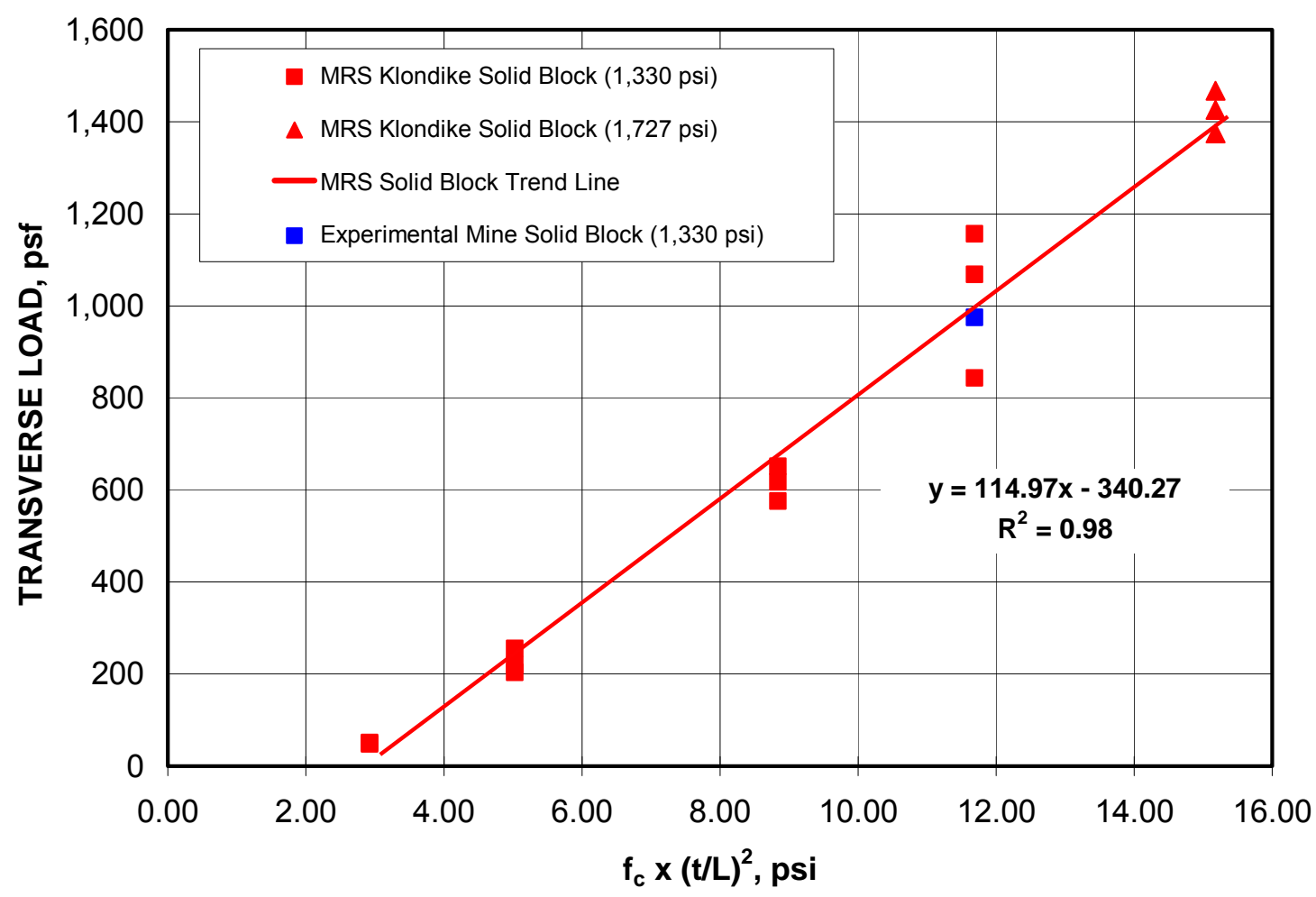

Figure 6-8. Comparison of full-scale mine test of Klondike conventional block with the MRS half-wall regression trend line and suite of MRS tests.

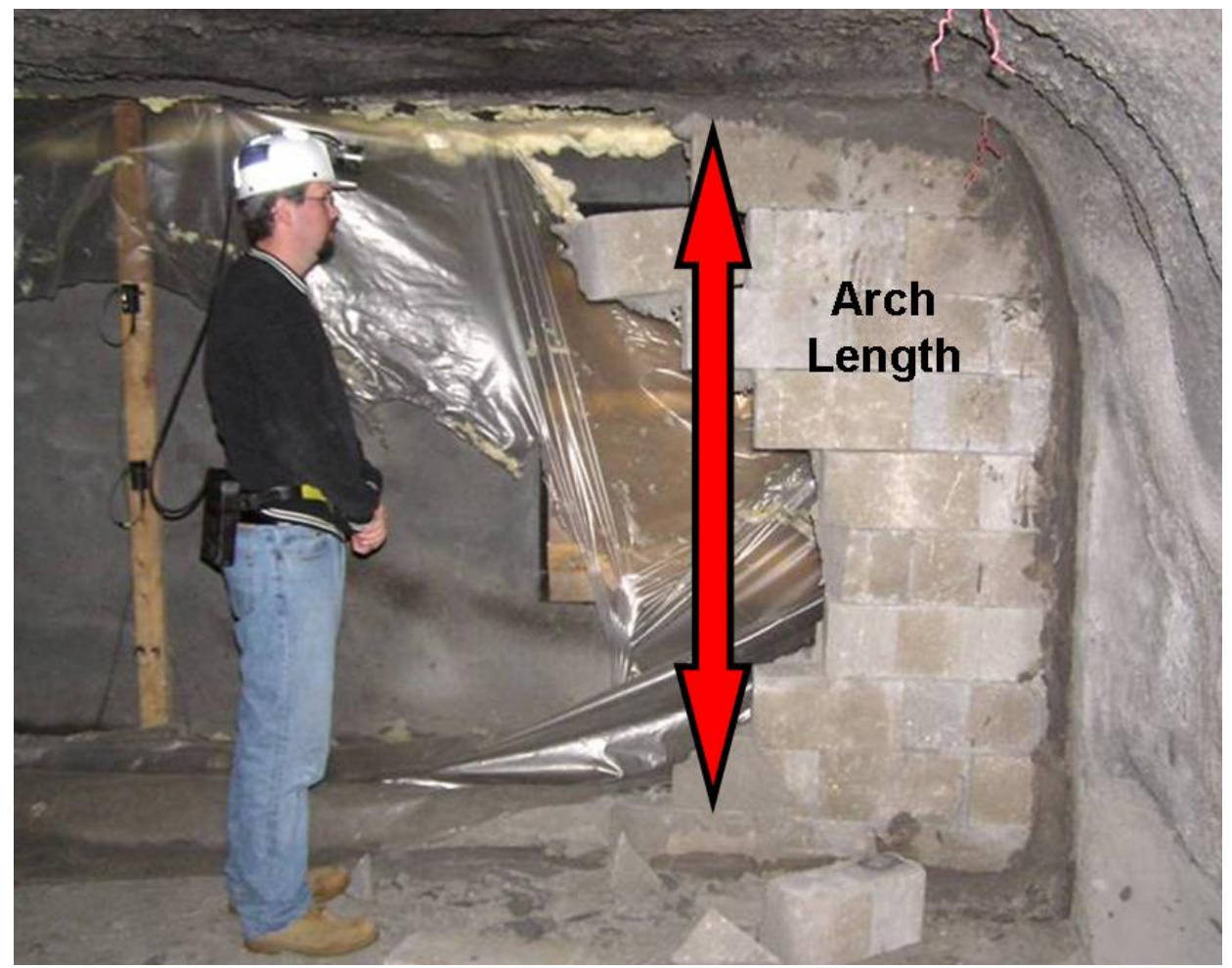

Figure 6-9. Photo showing conventional (Klondike) wall after full-scale test in the Experimental Coal Mine noting the arch length between the top and bottom course of block. 


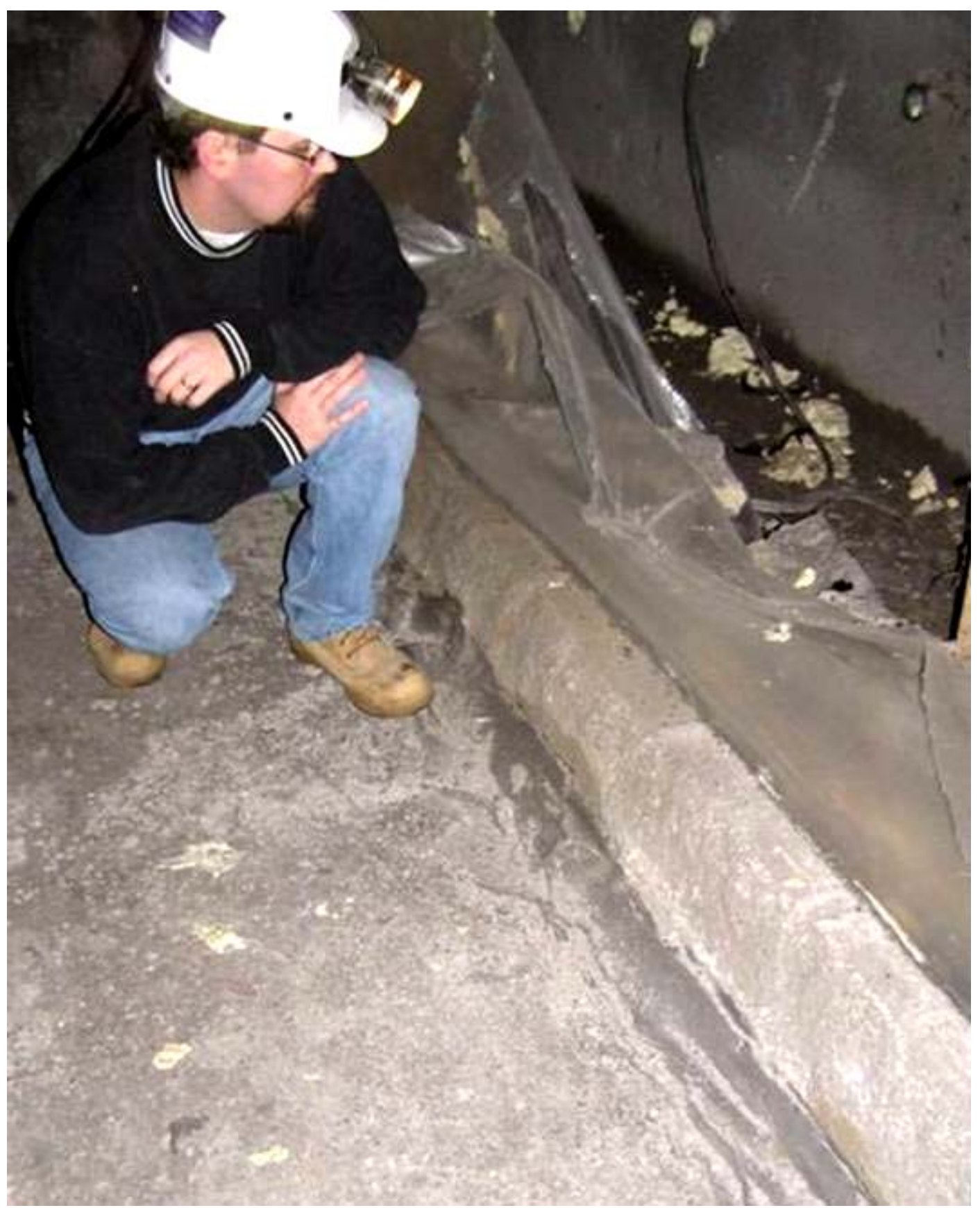

Figure 6-10. Photo showing bottom course of the conventional block wall after the test illustrating the crush zone and shearing action developed on the bottom course of block. 


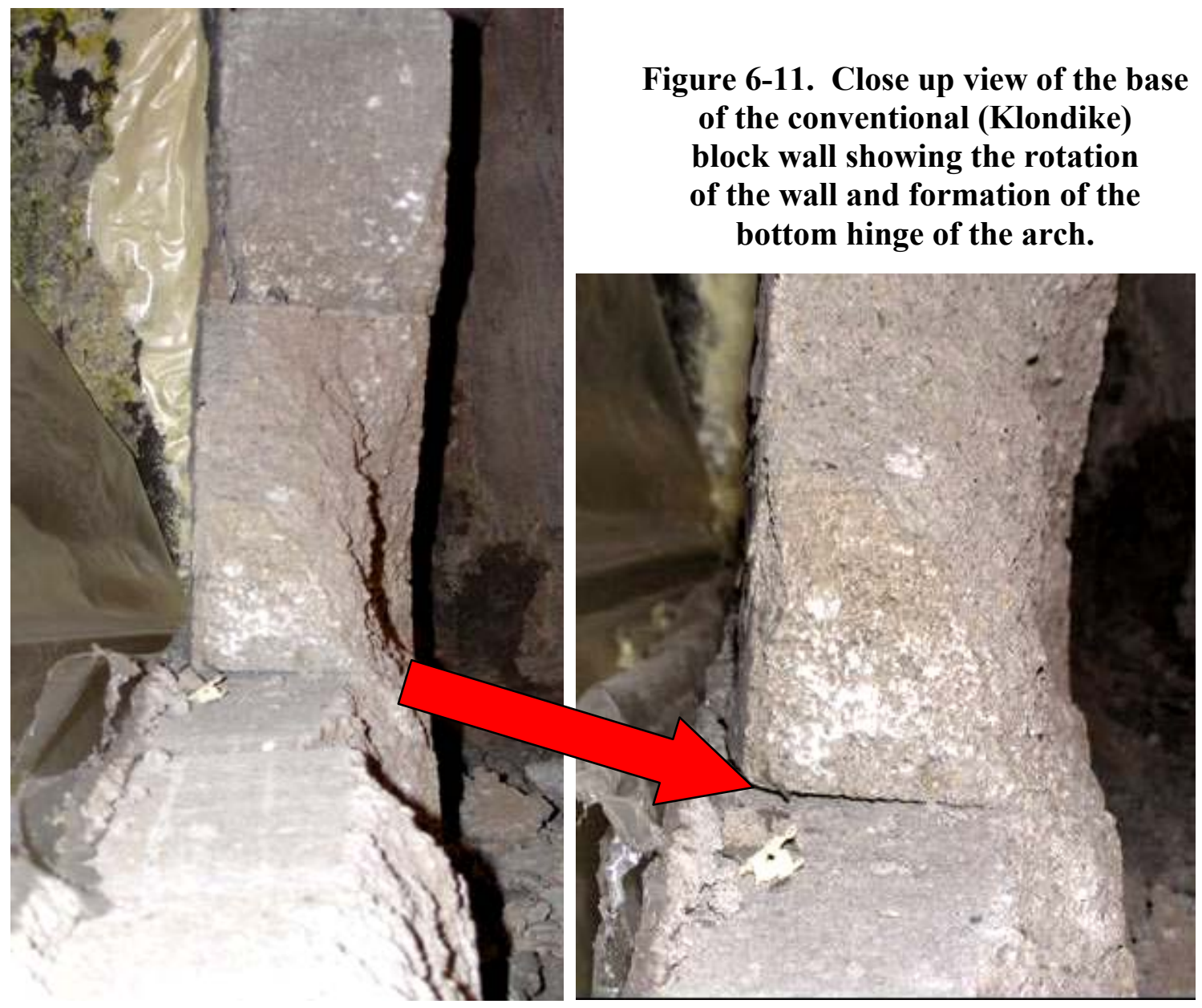

\subsection{NIOSH LAKE LYNN LABORATORY TESTS}

A series of tests were conducted at the NIOSH Lake Lynn Laboratory at the request of the Mine Safety and Health Administration (MSHA) to evaluate the effects of explosions on mine ventilation stoppings as part of NIOSH's research on the prevention and mitigation of gas/dust explosions. These tests also provided additional data to validate the transverse load capacity of stoppings. Lake Lynn Laboratory is located about 60 miles southeast of Pittsburgh, PA, and 10 miles northeast of Morgantown, WV. Underground workings are located in a massive limestone deposit; entries are sized to match those of commercial coal mines, thus making them true full-scale test galleries. Movable bulkheads permit the setup of single entry, triple entry, and longwall face configurations for experiments. 


\subsubsection{Explosive Charge Loading}

The stopping tests were conducted in the Longwall Gallery, which is illustrated in figure 6-12. The primary purpose of these experiments was to evaluate the post-failure conditions of the remains of a stopping that was destroyed by explosive pressures within the mine environment. The debris field was mapped to provide a qualitative assessment of the damage caused by the explosive charge. MSHA hopes to use this type of forensic secondary information to gain insight into determining the magnitude of explosions when they do occur in the mines and what might have led to such a catastrophic event.

The test protocol consisted of constructing stoppings in the crosscuts between the $\mathrm{C}$ and B-drifts of the Lake Lynn Longwall Gallery, and progressively increasing the intensity of gas/dust explosions to induce sufficient air pressures to cause transverse loading failures of the stoppings. Both hollow-core and solid concrete block stoppings were evaluated in this study. The hollow-core block had an average compressive strength of 1,456 psi and the solid block an average compressive strength of 1,900 psi. The stopping walls constructed in the crosscuts were 12 courses high $(7.5 \mathrm{ft}), 6$-inches thick, and approximately $20 \mathrm{ft}$ in length. Pressure transducers were used to measure both the static and dynamic pressure at the stoppings resulting from the explosive charge. A highspeed, PC-based data acquisition system collected the data from the various instruments at a sampling rate of 1,500 samples per sec. The results of this study were reported in an internal report by Weiss, Harteis, and Cashdollar (2004). The static pressure on the stopping from these tests is converted into psf units and is used to determine the transverse load capacity of these stopping constructions for comparison to the Mine Roof Simulator half-wall, rigid-arching tests.

\subsubsection{Test Results and Comparisons to Half-Wall MRS Tests}

The results of the explosion tests at the Lake Lynn Laboratory indicated that the transverse load capacity of the dry-stacked, hollow core stopping was 490 psf and 821 psf for the solid, dry-stacked concrete block stopping. The static pressure vs. time plots for both the hollow-core and solid block stopping are shown in figure 6-13a and figure 6$13 \mathrm{~b}$, respectively. Since neither of these particular blocks were available for testing at the MRS, a direct comparison to MRS half-wall tests could not be made. However, by 
computing the term $f_{\mathrm{c}} \times(\mathrm{t} / \mathrm{L})^{2}$, a comparison can be made to overall MRS test results by comparing this data point to the linear regression trend line developed from the MRS half-wall tests (figure 5-15). In this case, the wall height (L) that arched was based on 10 courses of block, two less than the actual wall height since the top and bottom course remained intact following the failure of the wall. For a nominal block height of 8 inches, this produces an arch height (L) equal to 80 inches. Using 6 inches for the wall thickness and 1,456 psi for hollow-block compressive strength, the $f_{\mathrm{c}} \times(\mathrm{t} / \mathrm{L})^{2}$ term for hollow-block wall equates to 8.19 psi. Using 1,900 psi for the block compressive strength with the same wall dimensions equates to 10.69 psi for the solid block wall.

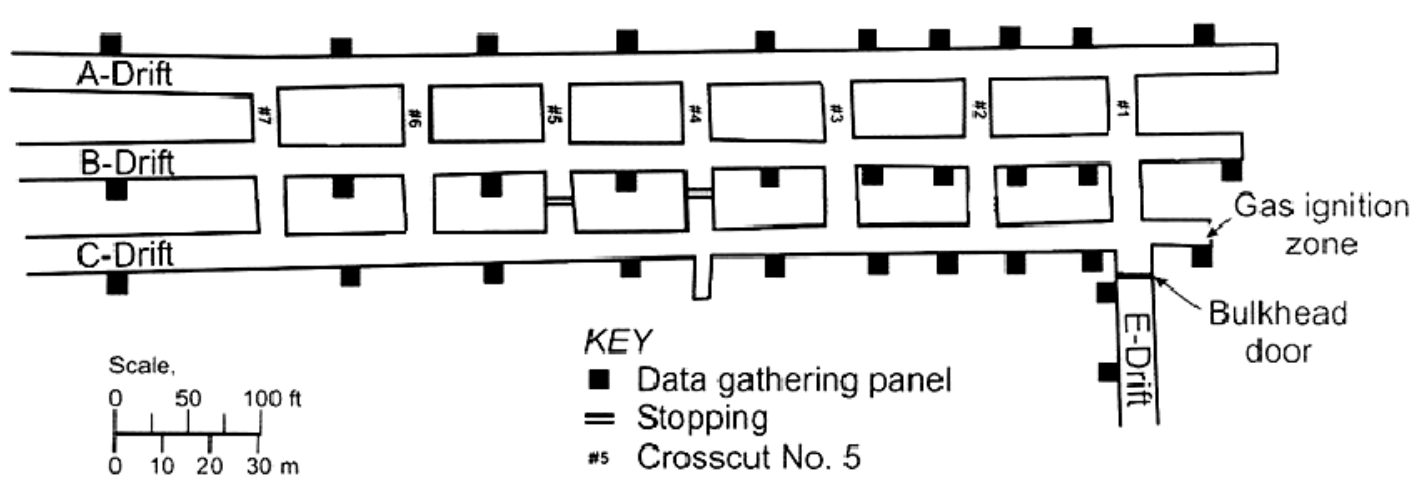

Figure 6-12. Diagram of the longwall gallery at the Lake Lynn limestone mine showing location of stopping constructions in the \#4 and \#5 crosscut. 


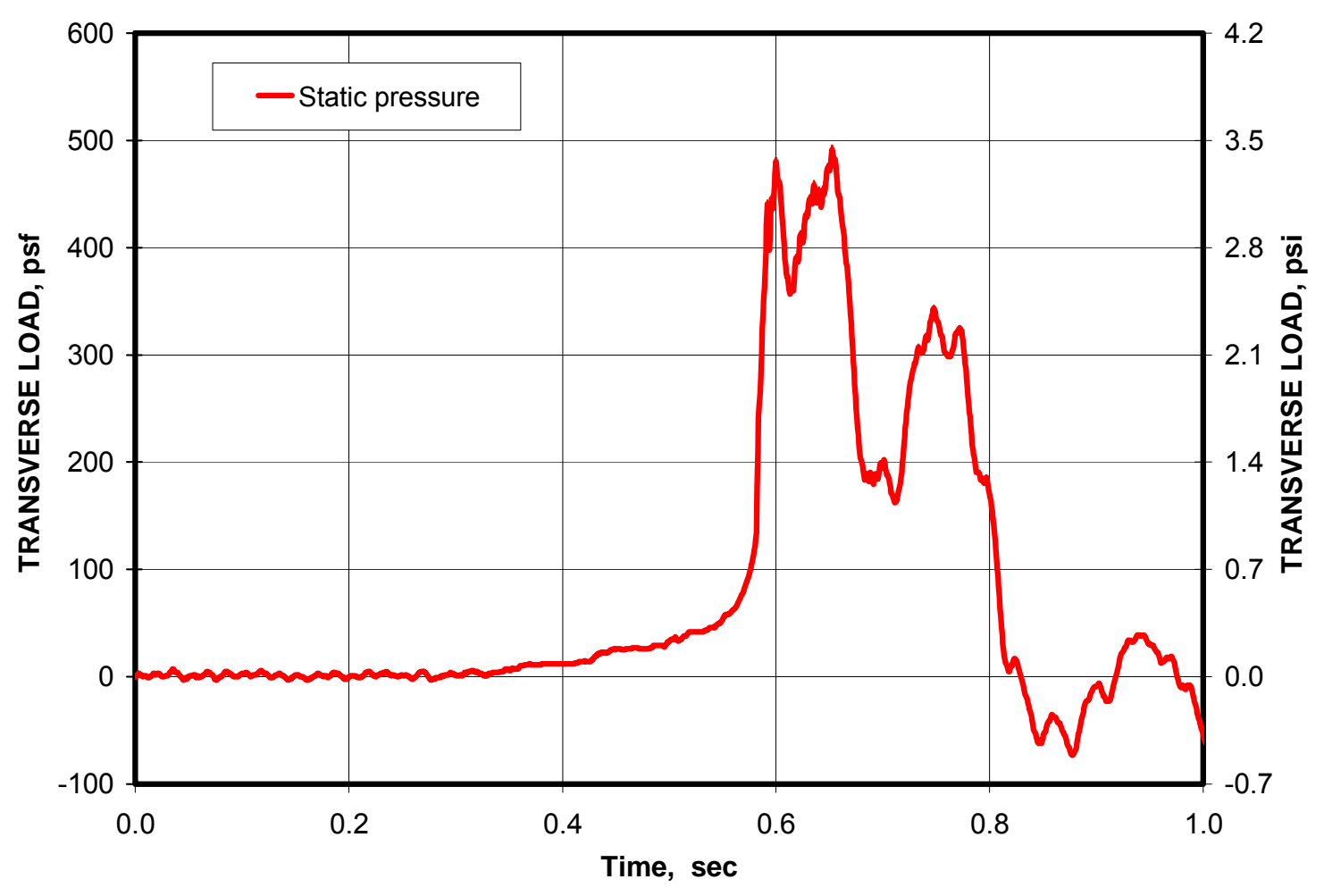

Figure 6-13a. Transverse load testing of a hollow-core stopping at the Lake Lynn Laboratory.

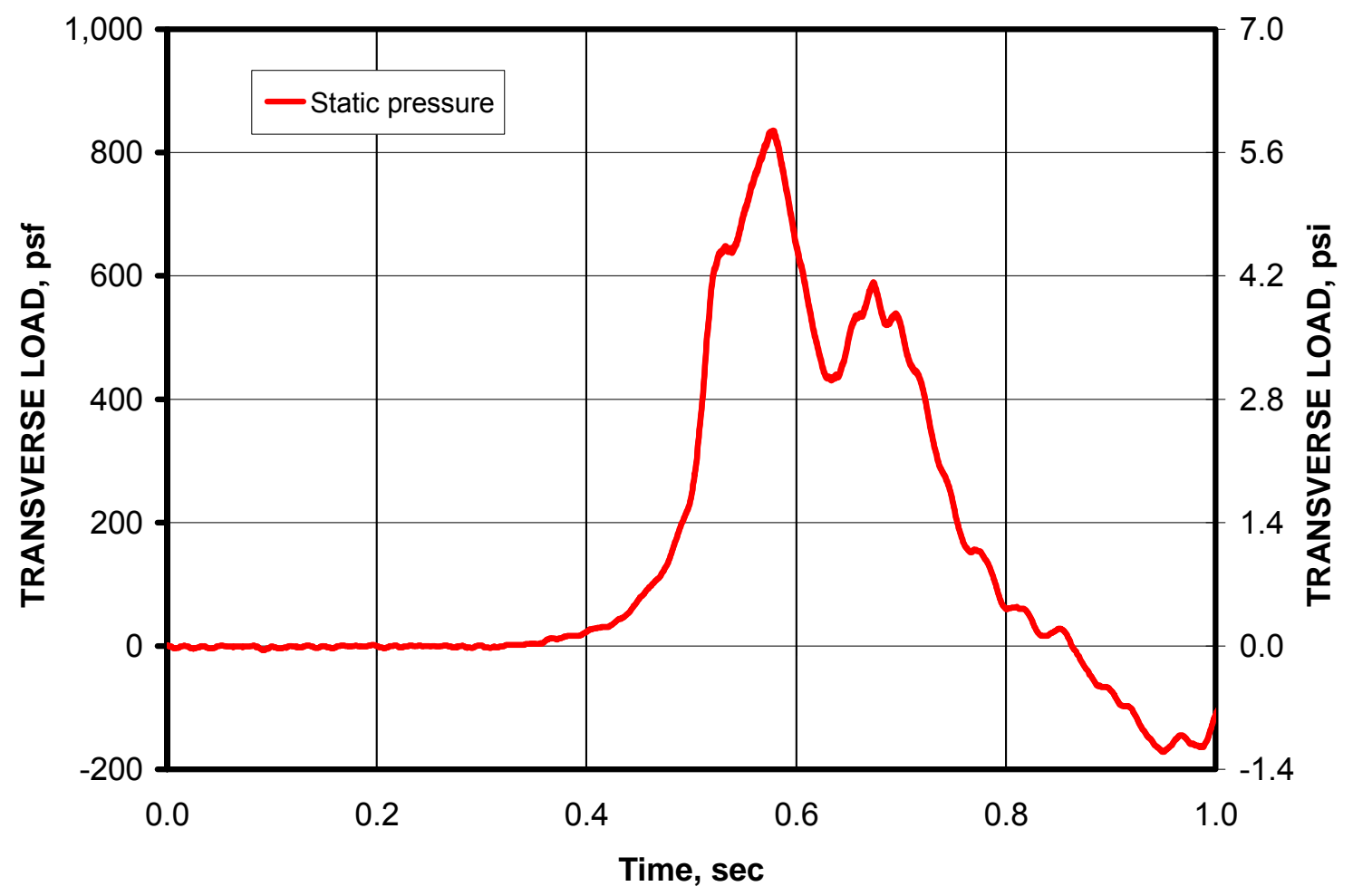

Figure 6-13b. Transverse load testing of a solid block stopping at the Lake Lynn Laboratory. 
These results are depicted in figure 6-14. As seen in figure 6-14, the Lake Lynn tests of the hollow-core and solid block stoppings are reasonably close to the regression line developed from the MRS rigid-arching tests. Both the hollow-core and solid block transverse load capacities measured in the full-scale Lake Lynn tests were lower than the MRS trend line prediction. The measured transverse load capacity of the hollow-core stopping was $22 \mathrm{pct}$ less than the MRS trend line and the solid block full-scale transverse load capacity was 9.8 pct lower. The latter is within the variability observed in the MRS testing. No hollow-core block tests were conducted in the MRS, the failure behavior of the hollow-core block may be sufficiently different to account for the larger disparity betwwen the hollow-core full-scale result and the MRS prediction based on solid block constructions.

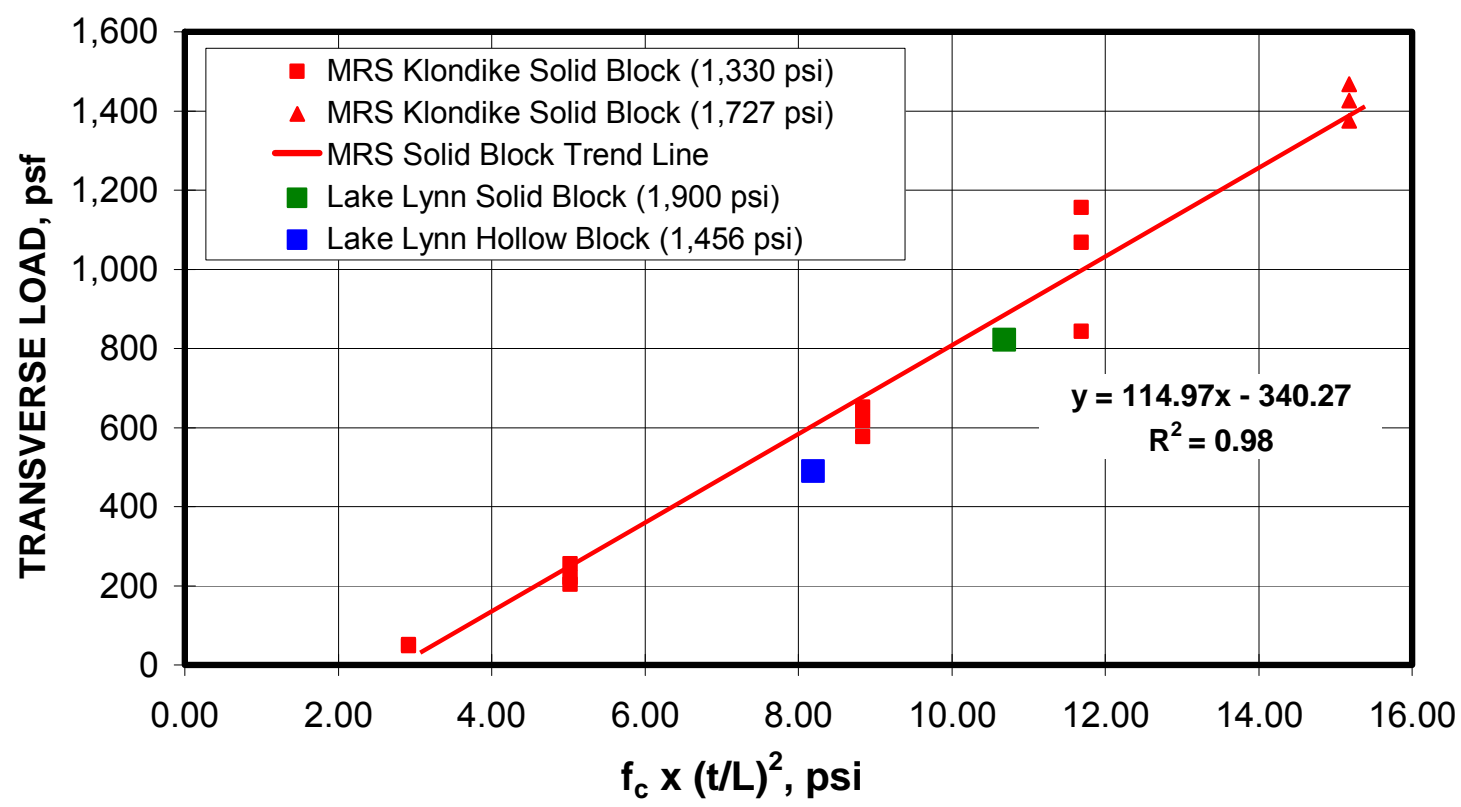

Figure 6-14. Comparison of Lake Lynn full-scale stopping tests with the MRS rigid-arching tests. 


\section{SUMMARY AND CONCLUSIONS}

Stoppings are an integral part of any underground mine ventilation system. In coal mines, stoppings may be either classified as temporary or permanent structures. Permanent stoppings are generally constructed from some form of concrete block, typically dry-stacked to form a wall, equal in thickness to the narrow dimension of the block, and bridging between the mine roof and floor and pillar ribs. The CFR specifies that stoppings must be able to withstand 39 psf of transverse loading on the face of the stopping in a freestanding loading condition to be suitable for coal mine use in the United States. This specification is based on ASTM E 72 testing requirements, which is intended for building construction of masonry panels and walls. The author does not believe that it provides an accurate representation of the loading conditions that occur in the mining situation. For dry-stacked stopping constructions, the transverse load capacity under the CFR criteria is primarily determined by the tensile strength of the sealant. Any block material, regardless of its physical properties, can be made to pass the acceptance test for use in underground coal mines provided the sealant is strong enough and can adhere to the surface of the block. As such, the 39-psf transverse load requirement is an irrelevant, arbitrary, and misleading performance measure that does not accurately correspond to the true transverse loading capability of stoppings in the mine.

The restraint provided by the mine roof and floor and coal pillars allow the stopping wall to arch between these abutments as the wall bends from the application of transverse loading. Arching has long been recognized as a valid loading mechanism that can dramatically improve the capability of jointed structures to resist loading induced by bending. Arching relies on compressive forces within the wall structure to offset the bending moment induced by the deflection of the wall from the application of transverse loading. For dry-stacked stopping constructions, which have no tensile strength across the joints except for the sealant on the face of the joint, these compressive forces can increase the transverse load capacity of a stopping by an order of magnitude or more.

Typically, transverse loading theoretically causes a three-hinge arch to form in a stopping wall as the joint running horizontally across the wall opens at the mid vertical span between the mine roof and floor. This creates two half-sections of wall, one above and 
one below the middle joint. Hinges also form at the mine roof and floor interface as the half-wall sections remain together as unit, but also try to rotate at the roof and floor interface. Vertical compressive forces are developed in the wall as the wall is restrained from horizontal rotation by the mine roof and floor. These compressive forces allow powerful force couples to be developed that control the transverse loading capacity of a jointed structure that otherwise would have very little capability to resist bending induced by transverse loading.

A static analysis of the arching condition for a stopping was examined to develop a theoretical relationship for predicting the transverse load capacity of a stopping. The analysis indicates that both the compressive strength $\left(f_{\mathrm{c}}\right)$ of the construction material and the thickness ( $t$ ) of the wall have a significant impact on the transverse load capacity of a stopping. Increasing the thickness of the wall will cause an increase in the force couple developed by the resultant compressive forces acting on the wall, and thereby increase its transverse loading capacity. On the other hand, increasing the height $(\mathrm{L})$ of the stopping will reduce the force couple and thereby reduce the transverse load capacity of a stopping. None of these factors are considered in the current CFR criteria for mine ventilation stoppings. A theoretical design equation to predict the transverse loading was developed using these factors mathematically expressed by the term $f_{\mathrm{c}}(\mathrm{t} / \mathrm{L})^{2}$.

A lab testing protocol to simulate rigid-arching of stopping walls by biaxial loading in the NIOSH Mine Roof Simulator was developed. This process is simulated in the MRS by testing a half-height section of wall. The wall is restrained vertically by the fixed vertical position of the load frame platens, thereby acting as rigid end restraints simulating the mine roof and floor. The lower platen is then moved laterally, causing the base of the wall to displace with the platen and causing the wall to rotate accordingly, similar to the three-hinge theory. Crush zones are created at corners of the half-wall in the areas where these two hinges would occur in a full-height wall. By measuring the lateral load applied to the wall by the simulator, the transverse load capacity of the wall can be determined. This load is normalized to the area of the wall to determine a transverse pressure in psf units equivalent to that used in the CFR specifications.

Tests were conducted in the MRS on two different block types using this testing protocol. The two block types were selected for study because of their different physical 
characteristics. One block was made from a low density, autoclaved concrete material (540 psi) while the other block was made from more conventional materials including Portland cement, sand, and aggregate (1,330 and 1,727 psi, respectively). Tests were conducted on several wall heights. Although stopping walls are typically constructed with the narrow side of the block for the wall thickness in order to minimize the number of block required to construct the stopping, tests were also made with wide-side construction to evaluate the impact of wall thickness. For these tests, the transverse load capacity ranged from 69 to $417 \mathrm{psf}$ for the lightweight block and 51 to 1,467 psf for the conventional block with wall heights ranging from 5 to $10 \mathrm{ft}$. Comparing these results to the 39-psf requirement in the current CFR, it clearly shows the disparity caused by the assumptions made in the boundary conditions, freestanding as considered in the CFR and the rigid arching conditions being proposed here as a more accurate representation of the actual in mine conditions. A regression line was fitted to the design equation developed from these laboratory tests showed a 98 pct correlation of the measured transverse load to the theoretical $f_{\mathrm{c}} \times(\mathrm{t} / \mathrm{L})^{2}$ term for both block types evaluated in this study.

The MRS half-wall rigid-arching tests were verified with two full-scale tests of stopping walls in the NIOSH Experimental Coal Mine at the Pittsburgh Research Laboratory and two full-scale tests at the NIOSH Lake Lynn Laboratory, which is also an underground mine. Overall, good agreement was shown between the MRS tests and the full-scale mine tests. Three of the four MRS test results were within 10 pct of the fullscale mine tests. A 22 pct difference in the design equation prediction from the MRS test compared to the full-scale test at the Lake Lynn Laboratory was observed for the fourth test. However, the full-scale wall in this particular test was constructed from hollow-core concrete block while the MRS design equation was based on solid concrete block. Although the hollow-core and solid concrete block are made from the same materials, the hollow-core block are more prone to shear failures and may require a separate design equation for a more accurate prediction of the transverse load capacity of hollow-core block stoppings. Separate design equations were developed for the lightweight block and the conventional concrete block used in the Experimental Coal Mine Tests. These tests showed the accuracy of the rigid arch design theory. 
In conclusion, rigid arching stopping design would be a radical departure from the current freestanding wall design required by the CFR. The physical properties of the block and the size of the mine opening would need to be examined to determine the proper design for a stopping application. The sealant would no longer be considered to impact the transverse load capability of the stopping. Since the actual transverse load capacity of the stopping can be determined, the stopping can be designed based on the required transverse load capacity for a specific set of conditions in the mine, as opposed to the current system that permits stoppings of widely ranging transverse loading capabilities to be employed in the same environment. This approach should lead to a safer mine environment for the tens of thousands of mineworkers in underground coal mines.

This thesis has shown that rigid arching is an accurate loading mechanism to evaluate the true transverse load capacity of mine ventilation stoppings. Furthermore, the protocol developed in the MRS has been shown to accurately predict full-scale behavior of an actual stopping in a true mine environment. Using this protocol, a full research program can be undertaken to evaluate the design parameters relevant to transverse load capacities for stoppings. This will include a systematic evaluation of these parameters for all the various block types that are currently on the market. From this systematic study, transverse load design equations for each block type can developed. 


\section{FUTURE RESEARCH RECOMMENDATIONS}

Another important factor to consider that also is ignored in the current CFR requirements is that of axial loading induced by roof-to-floor ground movements. Up to a point, axial loading can enhance the transverse loading of a stopping considerably. At some point, the wall will be sufficiently damaged from the axial loading and the arching capability will be degraded. Another factor that needs to be evaluated in this regard is the impact of strain softening materials that are used to reduce the stiffness of the wall so it can absorb ground deformations without causing damage to the wall. However, such materials are also likely to significantly degrade the arching and transverse loading capability of the stopping. What is needed is a unified approach that addresses both the transverse loading and ground deformations issues to design an effective stopping for a particular set of conditions. The author hopes to pursue this work in a Ph.D. dissertation in the near future. 


\section{BIBLIOGRAPHY}

1. Anderson C. 1984. Arching Action in Transverse Laterally Loaded Masonry Wall Panels. The Structural Engineer, vol. 62B, no. 1, March, 12 pp.

2. ASTM Designation E 72-80. 1981. Standard Methods of Conducting Strength Tests for Building Construction. Annual Book of ASTM Standards, Vol. 04.07, March, pp. 283, 293.

3. British Standards Institution. 1978. Code of Practice for Use of Masonry: Part 1 Structural Use of Unreinforced Masonry. BS 5628, BSI, London.

4. Code of Federal Regulations, Title 30 Mineral Resources, Part 75 Mandatory Safety Standards - Underground Coal Mines, Subpart D - Ventilation, Section 75.333 Ventilation Controls, pp. 471-473.

5. Cranston, W. B. and J. J. Roberts. 1976. The Structural Behavior of Concrete Masonry -Reinforced and Unreinforced. The Structural Engineer, vol. 54, no. 11, November, pp. 423-436.

6. Drysdale, R. G., A. A. Hamid and L. R Baker. 1994. Masonry Structures: Behavior and Design. Englewood Cliffs, NJ, Prentice Hall, ISBN: 0135620260.

7. Gillies, A. D. S., R. D. Pearson, R. Day, P. Dux and A. R. Green. 2001. Testing and Usage of Ventilation Control Devices within the Australian Coal Mining Industry. Proceedings of the 7th International Mine Ventilation Congress, Chapter 80, Cracow, Poland, June 17-22, pp. 565-572.

8. Kawenski, E. M. and D. W. Mitchell. 1966. Evaluation of Materials for Ventilation Structures. Mining Congress Journal, March, pp. 49-53.

9. Masonry Designer's Guide: Based on Building Code Requirements for Masonry Structures (ACI 530-92/ASCE, 5-92/TMS 402-92) and Specifications for Masonry Structures (ACI 530.1-92/ASCE 6-93/TMS 602-92). John H. Matthys editor.

10. Massachusetts Institute of Technology. 1954. Behavior of Wall Panels Under Static and Dynamic Loads II. Department of Civil and Sanitary Engineering, January. 
11. McDowell, E. L., K. E. McKee and E. Sevin. 1956. Arching Action Theory of Masonry Walls. Journal of the Structural Division, Proceedings of the American Society of Civil Engineers, vol. 82, no ST2, Paper 915, March, pp. 915-1 - 915-18.

12. Sapko, M. J, E. S. Weiss and S. P. Harteis. 2003. Alternative Methodologies for Evaluating Explosion-Resistant Mine Ventilation Seals. Published in the $30^{\text {th }}$ International Conference of Safety in Mines Research Institutes, South African Institute of Mining and Metallurgy.

13. Tien, J. 1996. Air Leakage Costs. A Strong Stopping Maintenance Program Saves Money and Gets More Air to the Face. Coal Age, Sept., pp. 88-121.

14. Weiss, E. S., S. P. Harteis and K. L. Cashdollar. 2003. NIOSH-PRL Evaluation of the Kennedy and Solid-Concrete-Block Stopping Designs for MSHA during LLEM Explosion Tests, September-November 2003. NIOSH Internal Report, March, $13 \mathrm{pp}$.

15. Weiss, E. S., S. P. Harteis and K. L. Cashdollar. 2004. NIOSH-PRL Evaluation of the Kennedy and Solid-Concrete-Block Stopping Designs for MSHA during LLEM Explosion Tests, Sept., 40 pp. 


\section{APPENDIX A - IMPACT OF THE WEIGHT OF THE BLOCK ON THE TRANSVERSE LOAD CAPACITY OF A FREESTANDING WALL}

The failure mechanism will be buckling of the wall, typically at the first joint below the top of the wall. The transverse loading that causes this failure mechanism can be determined from equation A.1.

$$
P=\frac{2 \times f_{a} \times A_{n} \times t}{h^{2}}
$$

Where $\mathrm{P}=$ Transverse load, psf(lbs per square foot),

$f_{\mathrm{a}}=$ Compressive stress at base of wall due to the weight of the block, psf,

$\mathrm{A}_{\mathrm{n}}=$ Area of wall per unit length, $\mathrm{ft}^{2} /$ lineal foot,

$\mathrm{t}=$ thickness of wall, $\mathrm{ft}$,

$\mathrm{h}=$ height of wall, ft.

The compressive stress at the base of the wall due to the weight of the block can be computed either if the weight of an individual block is known or if the density of the material is known. If the density is known, then compressive stress produced by the weight of the block is computed from equation A.2. If the block weight and dimensions are known, then the density of the material can be computed by dividing the weight by the volume of the block.

$$
f_{a}=\text { Density } \times h
$$

Where $\quad f_{\mathrm{a}}=$ Compressive stress at base of wall due to the weight of the block, psf, $\mathrm{h}=$ height of the wall, $\mathrm{ft}$, Density $=$ Density of the block material, $\mathrm{lbs} / \mathrm{cu} \mathrm{ft}$.

Using this analysis, the transverse loading capability of several block wall constructions are shown in figure A-1. The standard concrete masonry units (CMU) weigh approximately $50 \mathrm{lbs}$ each. These are among the heaviest block used, as shown in 
figure A-1, the weight of these block would provide for about seven psf of transverse load capacity to a dry-stacked block stopping. It is seen from this analysis that the weight of the block alone does not provide sufficient moment resistance to meet the 39 psf of transverse load currently required in the CFR.

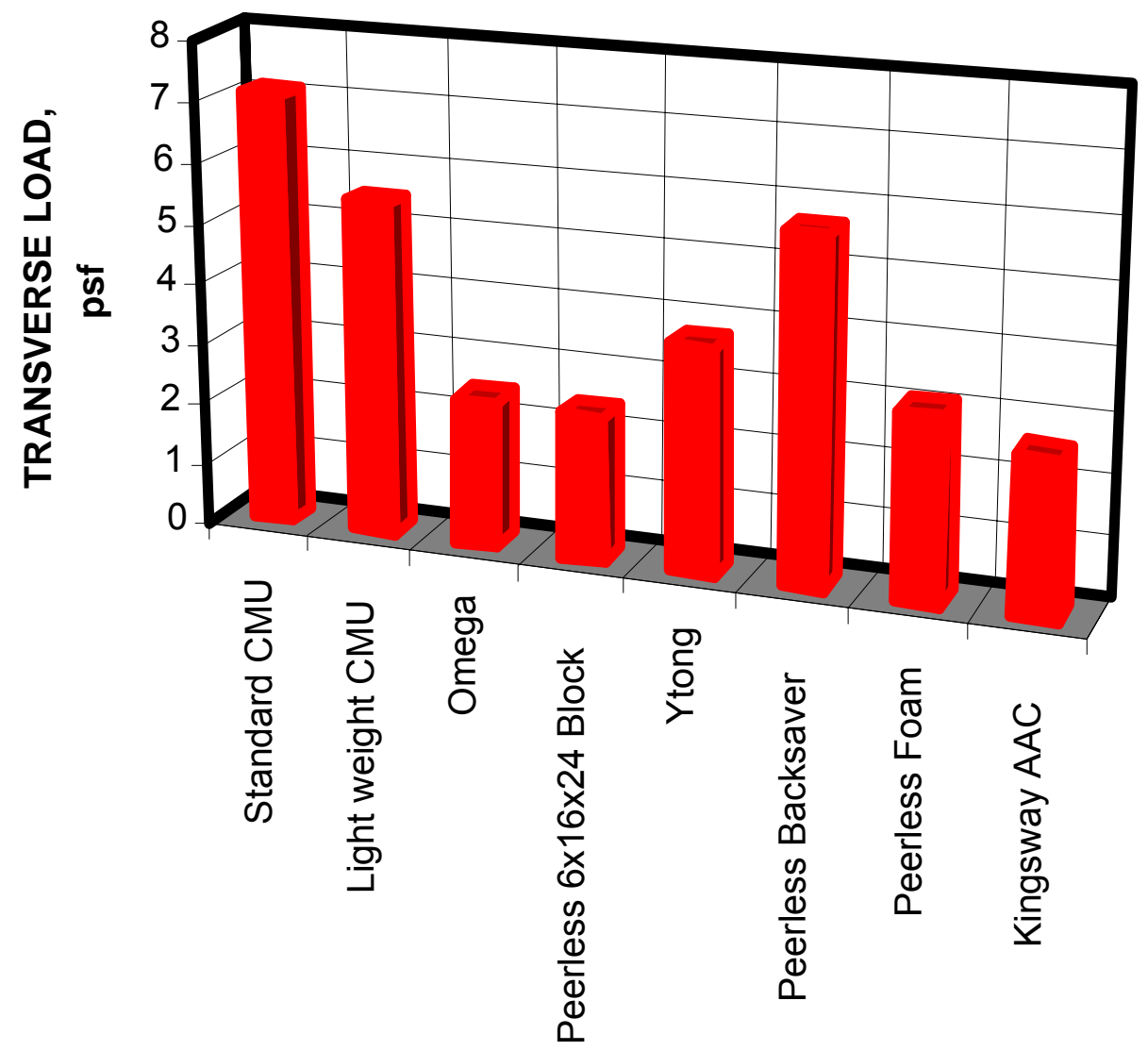

Figure A-1. Transverse load capacity due to weight of the block. 


\section{APPENDIX B - DESCRIPTION OF THE MINE ROOF SIMULATOR}

The Mine Roof Simulator (MRS) is a servo-controlled hydraulic press custom built by MTS Systems Corporation to U.S. Bureau of Mines (USBM) specifications. The simulator was built in 1979 at a cost of $\$ 7.5$ million. It was designed specifically for longwall shield testing, and is the only active load frame in the United States that can accommodate full-size shields. However, its size and unique capabilities provides a facility for testing a wide variety of large-scale structures, including various forms of standing roof support structures and mine ventilation stoppings.

A functional diagram of the load frame is shown in figure B-1. The load frame has several distinctive characteristics. The size of the upper and lower platen is $20 \mathrm{ft} \times 20 \mathrm{ft}$. The upper platen can be moved up or down and hydraulically clamped into a fixed position on the directional columns to establish a height for testing. With a maximum vertical opening between the upper and lower platen of $16-\mathrm{ft}$, the load frame can accommodate the largest shields currently in use. Load application is provided by controlled movement of the lower platen, operating in either force of displacement control. The load frame is a biaxial frame, capable of applying both vertical and horizontal loads. Load actuators are equipped with special hydrostatic slip bearings to permit simultaneous load and travel. This allows vertical and horizontal loads to be

applied simultaneously. The capability to provide controlled loading simultaneously in two orthogonal directions is unique at this scale.

Vertical loading is provided by a set of four actuators, one on each of the corners of the lower platen. Loads of up to 3 million pounds can be applied in the vertical direction by upward movement of the lower platen. Each actuator is capable of applying the full 3 million pounds of force, so that the specimen can be placed anywhere on the platen surface and the full 3 million pound capacity can be provided. The vertical (upward) range of motion of the lower platen is 24 inches.

Horizontal loading is also provided by four actuators, with two actuators located on both the left and right side of the load frame just below the floor level. These actuators act in pairs to provide horizontal displacement of the lower platen in either a positive or a 
negative (x) direction, reacting off the corner columns of the load frame. The horizontal range of motion of the lower platen is $16 \mathrm{in}$.

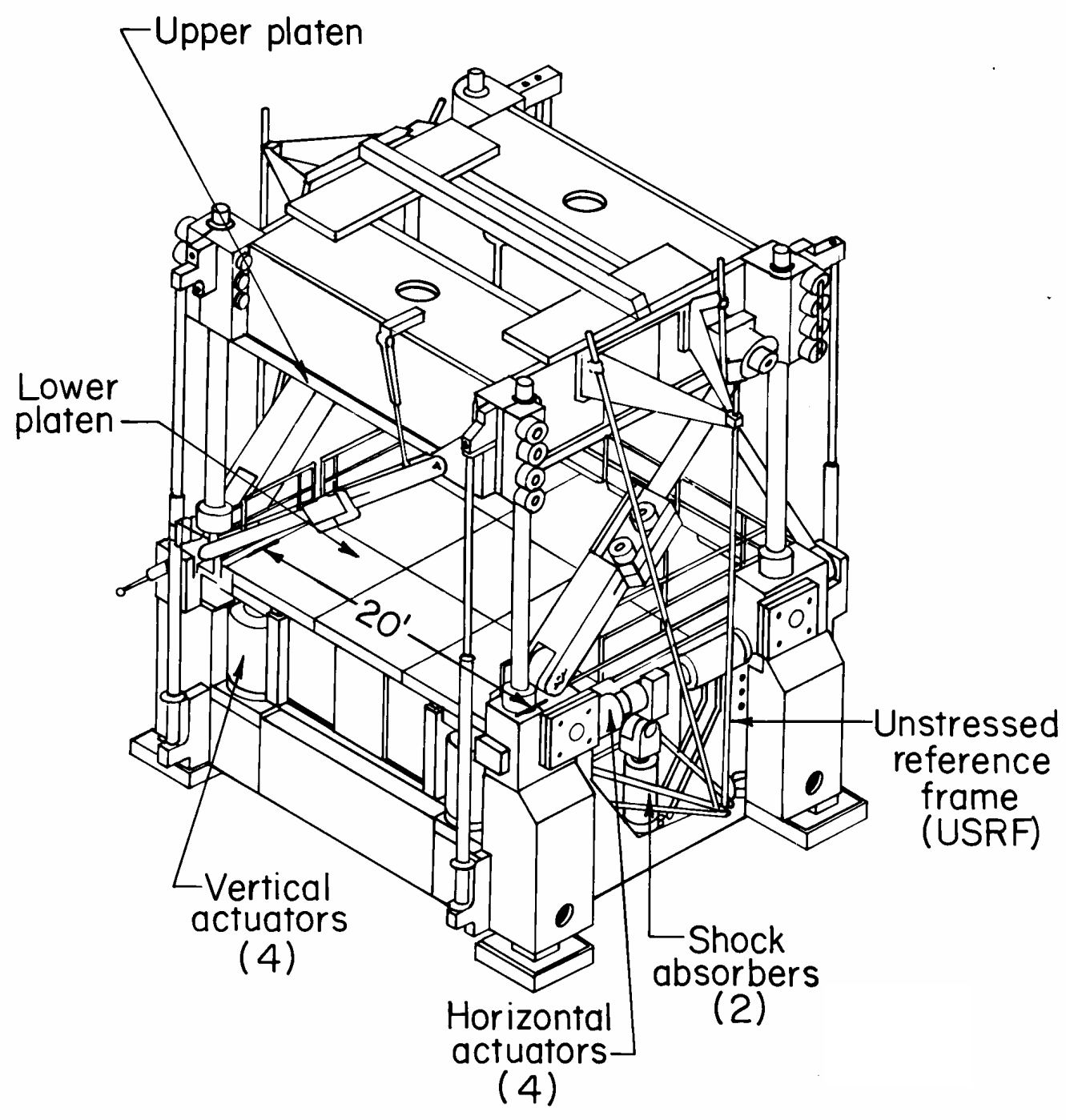

Figure B-1 Diagram of the Mine Roof Simulator.

There is no programmable control of the lower platen in the lateral horizontal axis (y-direction). The load frame has a reactive capacity of 1.6 million pounds in this direction, but loads cannot be applied in the lateral direction. The range of motion of the lower platen in this direction is $\pm 0.5 \mathrm{in}$. 
Six degrees of freedom control of the lower platen are provided by the unstressed reference frame, which provides feedback on platen displacements and rotations to the closed-loop control system. Pitch, yaw, and roll of the lower platen are controlled to keep the lower and upper platens parallel during load application.

A shock absorber actuator is positioned on the left and right side of the lower platen. These shock absorbers will control the displacement of the lower platen to less than 0.1 in in the event of sudden failure of the support specimen. This system absorbs energy stored in the load frame to maintain control of the platen and to avoid releasing stored energy into the specimen immediately following an abrupt specimen failure.

Two hydraulic pumps provide up to $3,000 \mathrm{psi}$ of pressure to the vertical and horizontal actuators during load application. The rate of movement of the lower platen is limited by the 140-gpm capacity of the hydraulic pumps. The maximum platen velocity assuming simultaneous vertical and horizontal displacement is 5.0 inches per minute. 


\section{APPENDIX C - TEST RESULTS FROM MRS HALF-WALL RIGID ARCHING STUDIES}
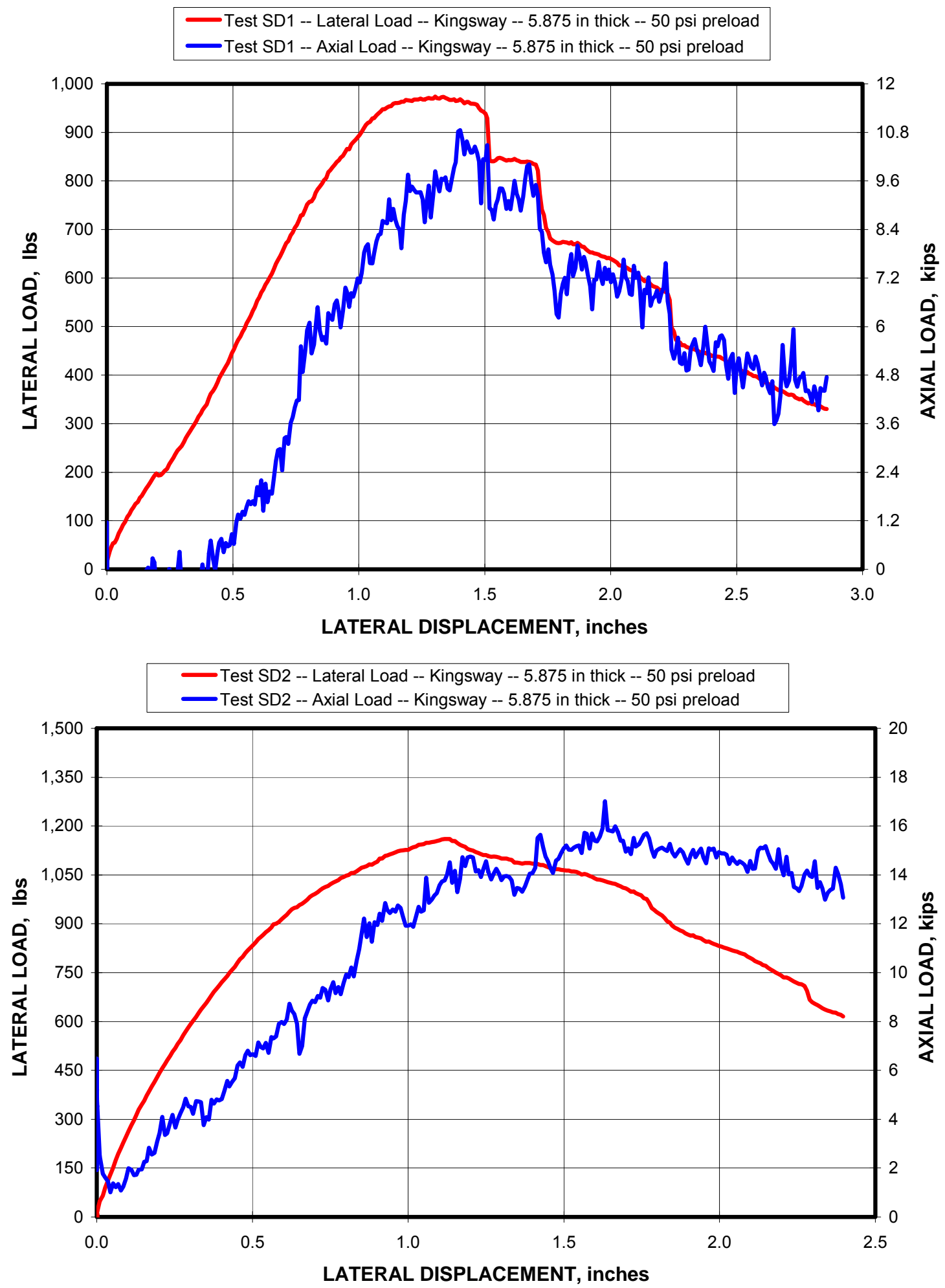

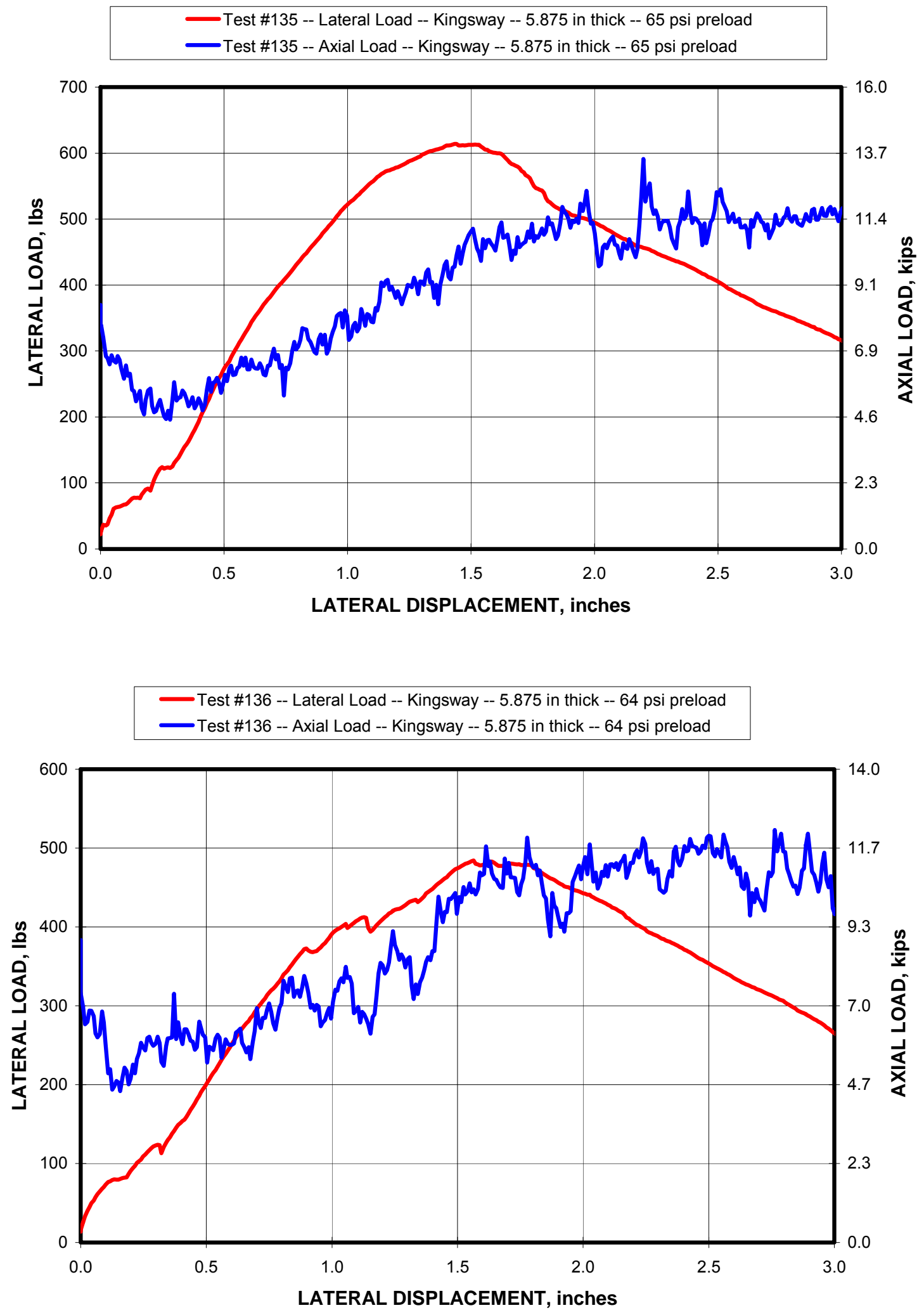

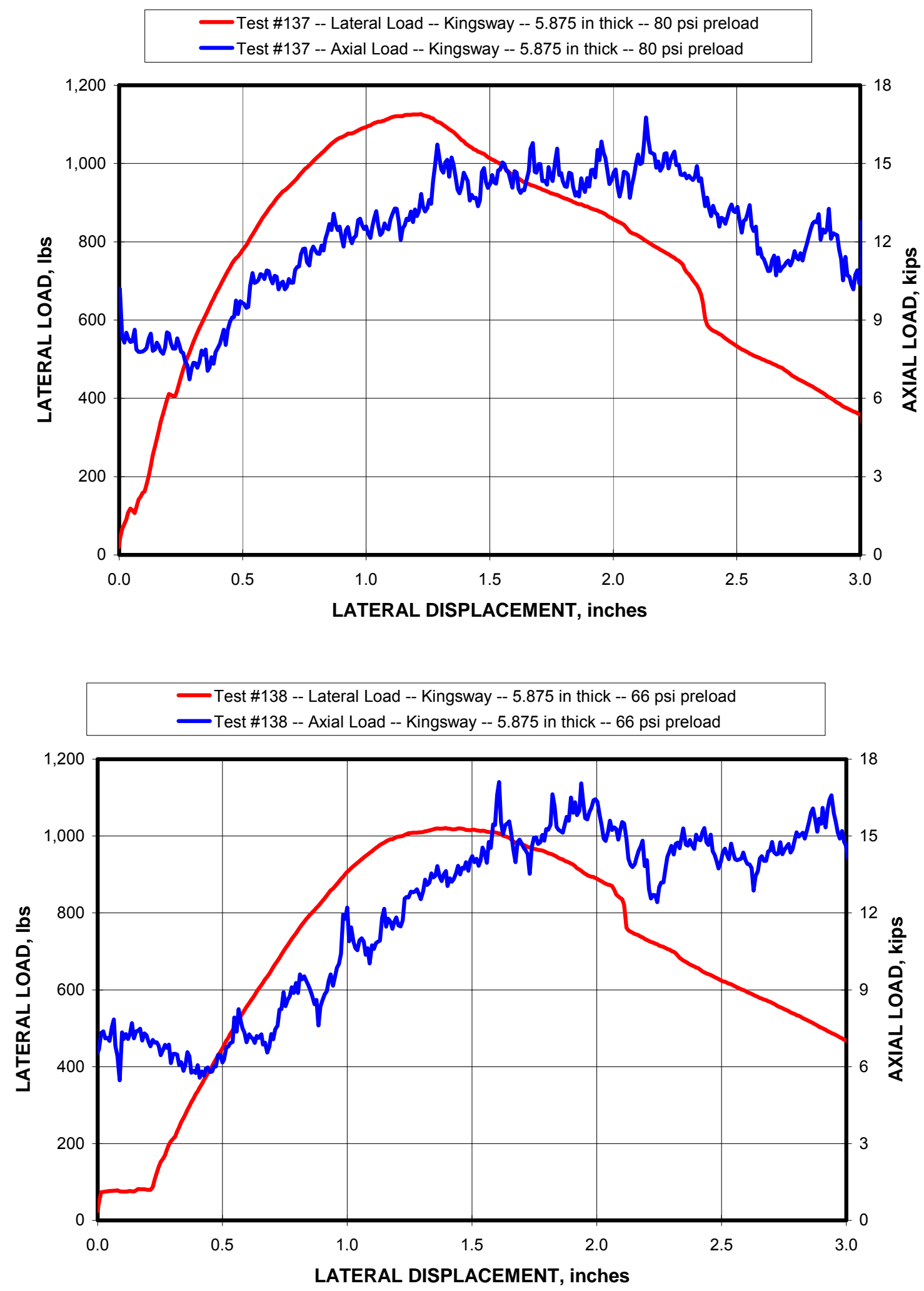

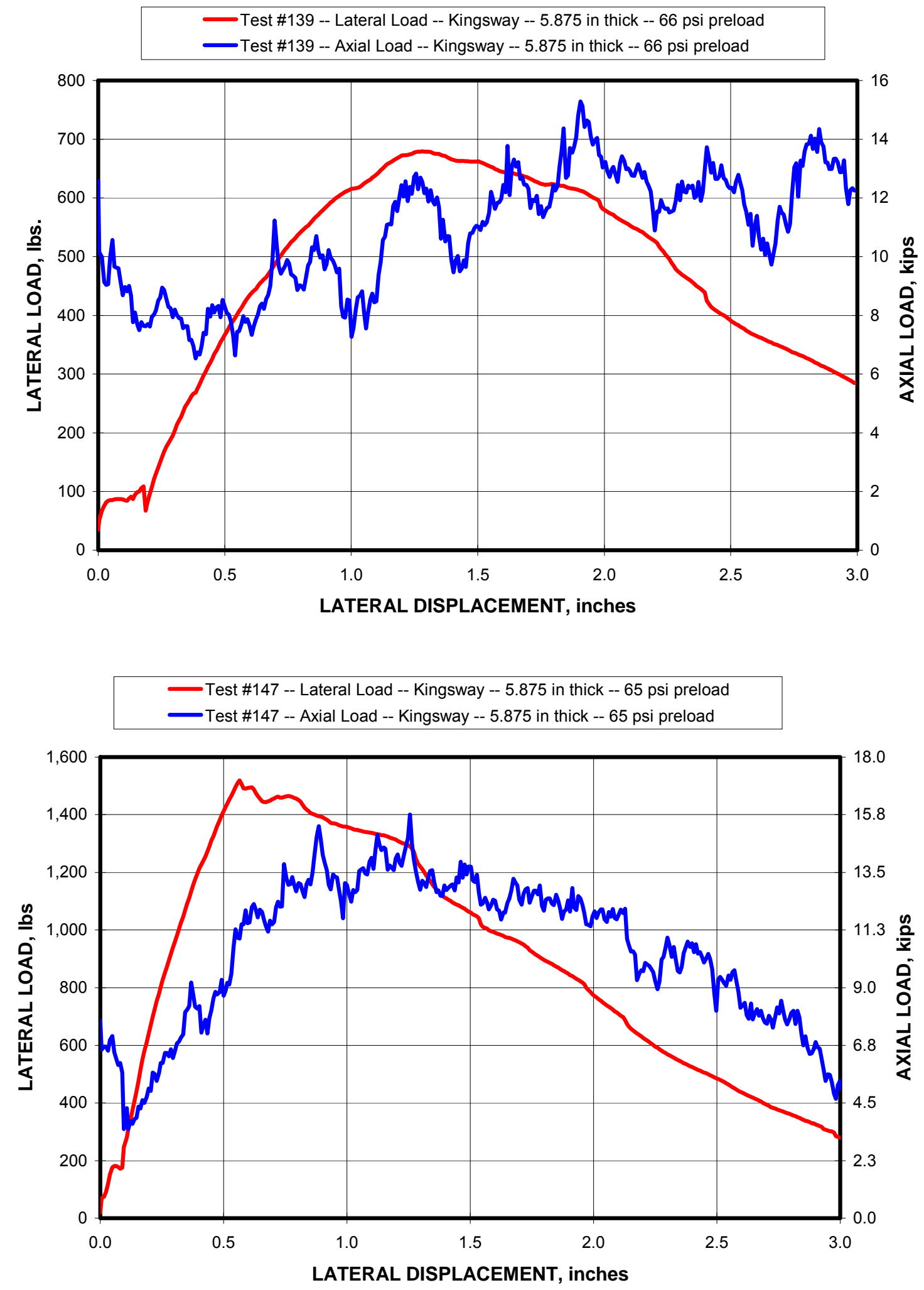

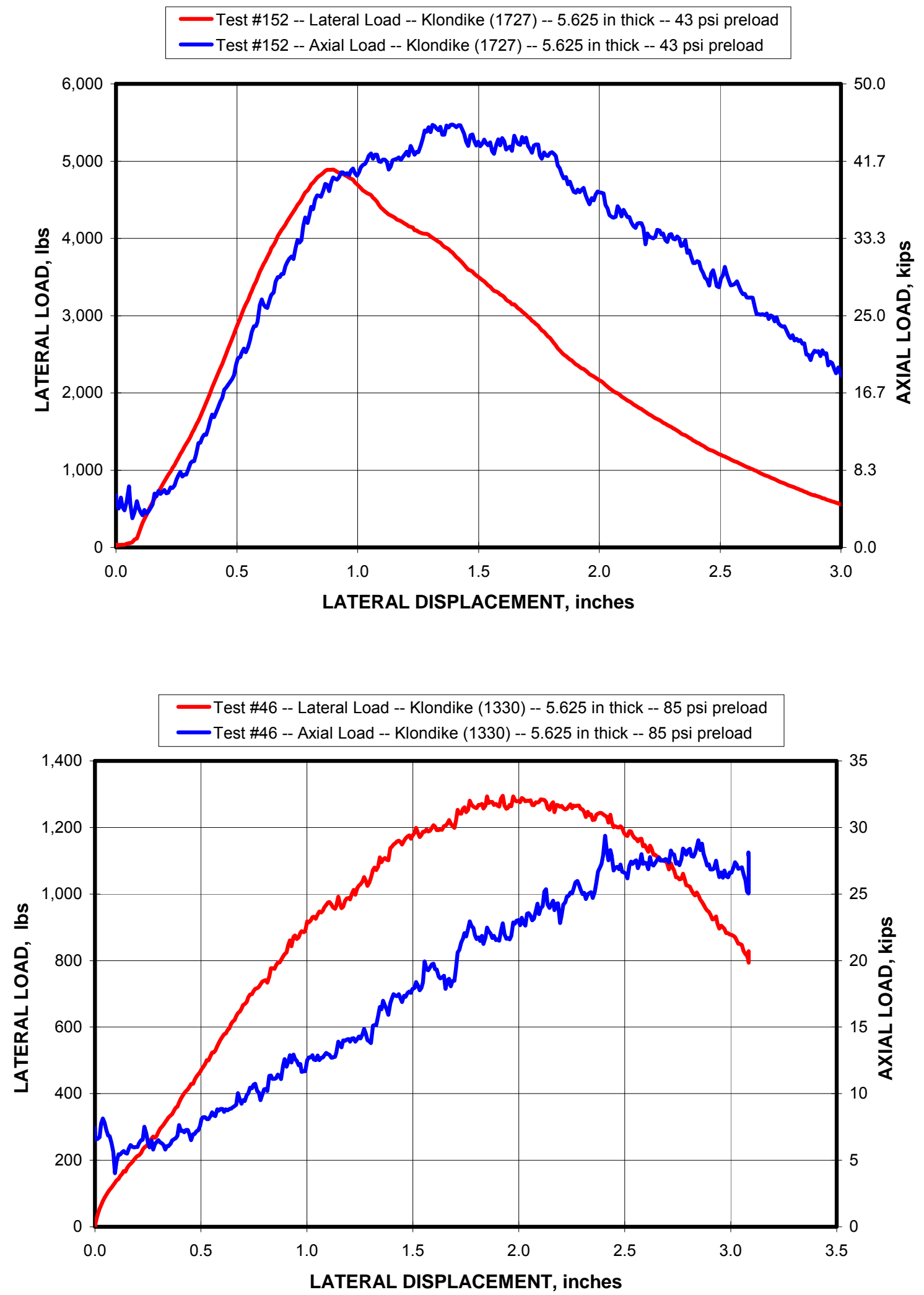

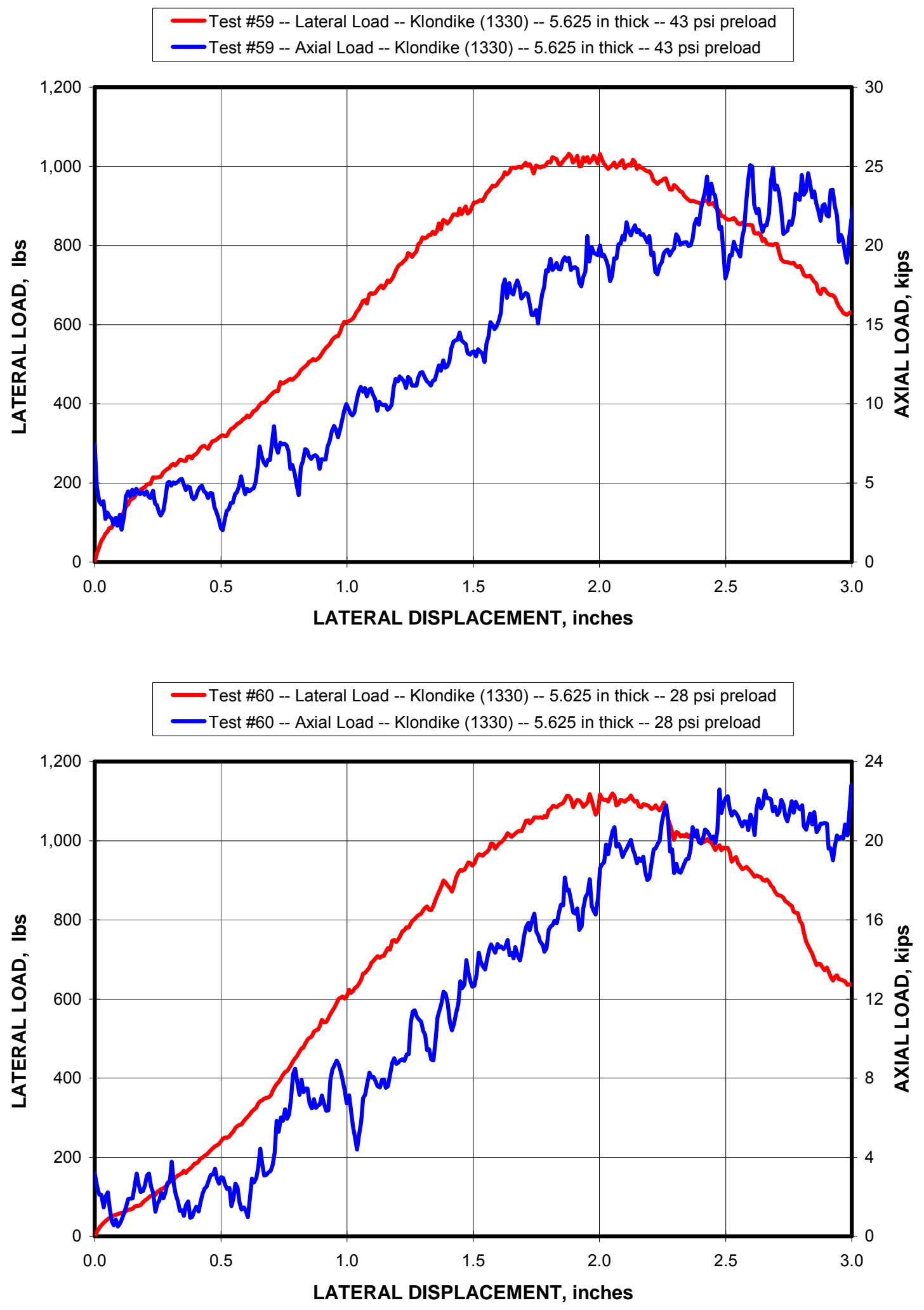

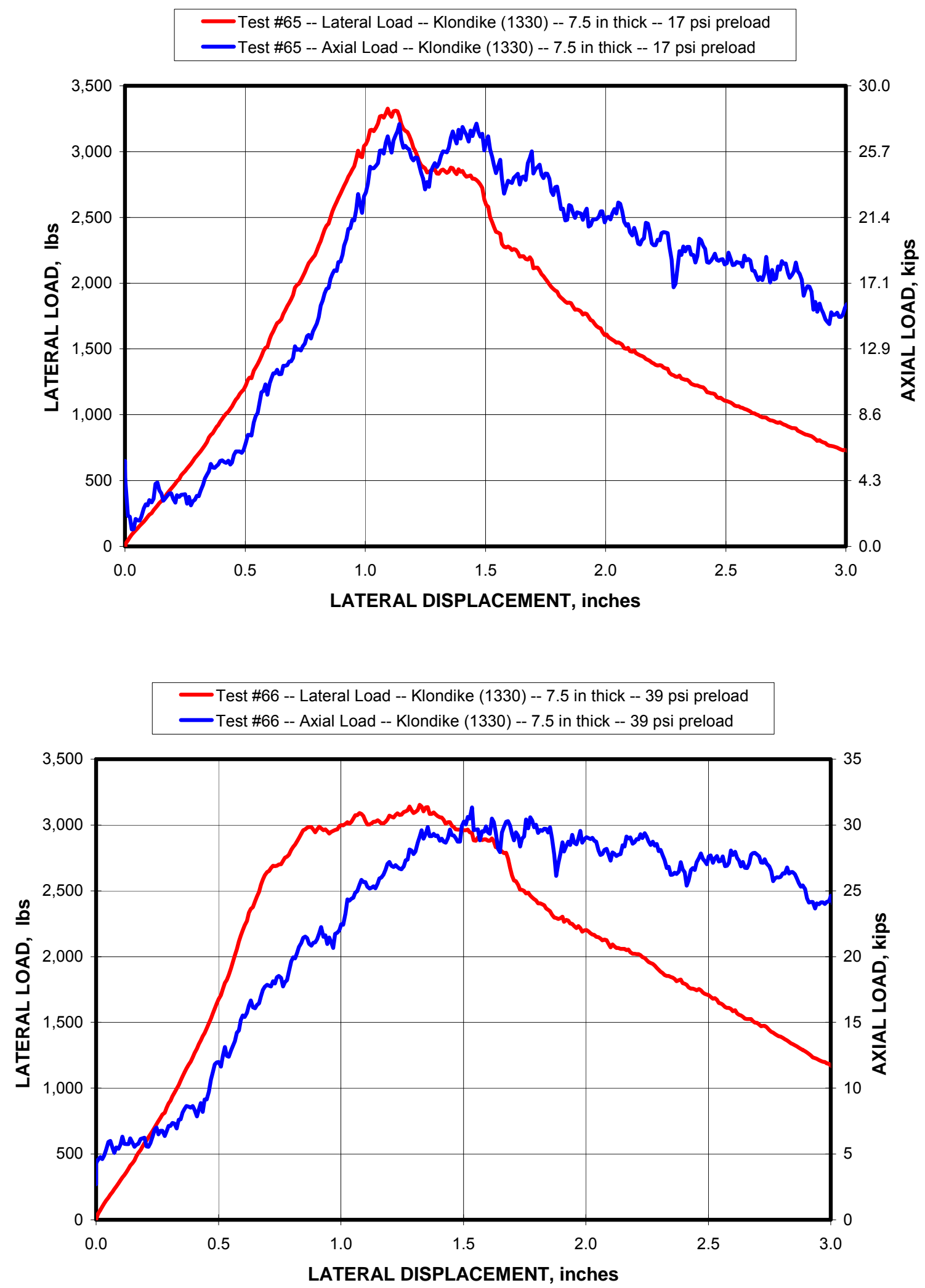

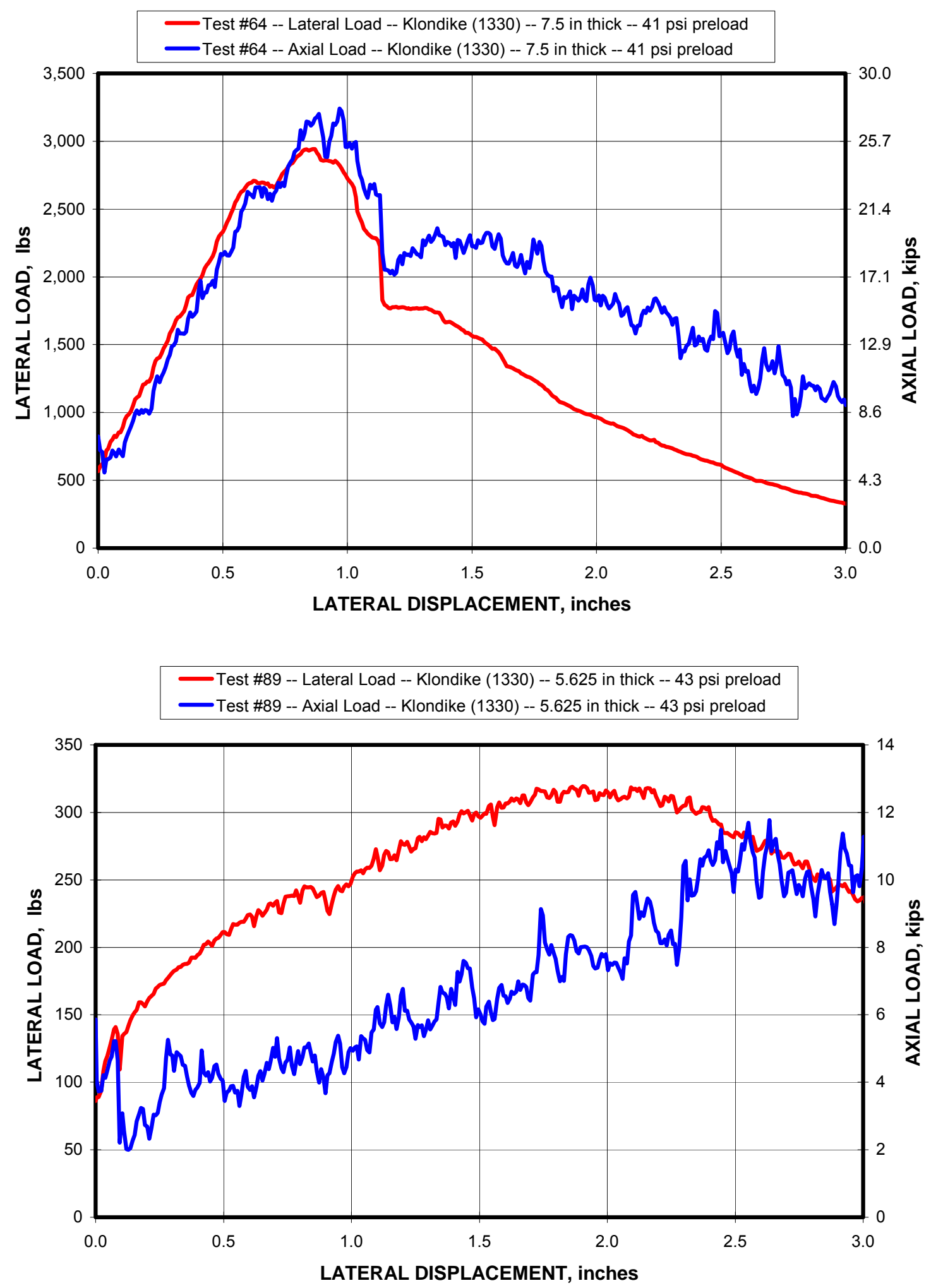

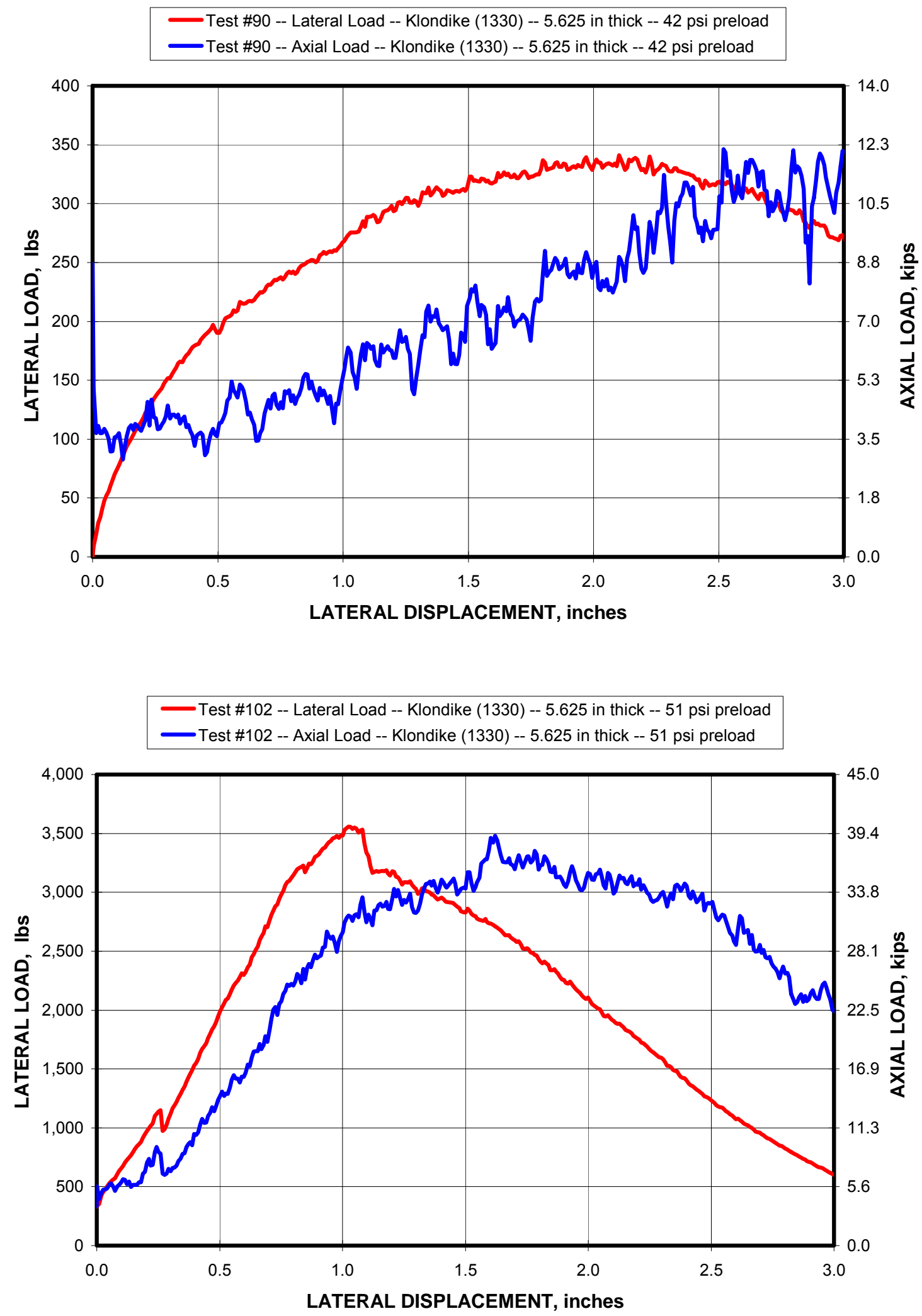

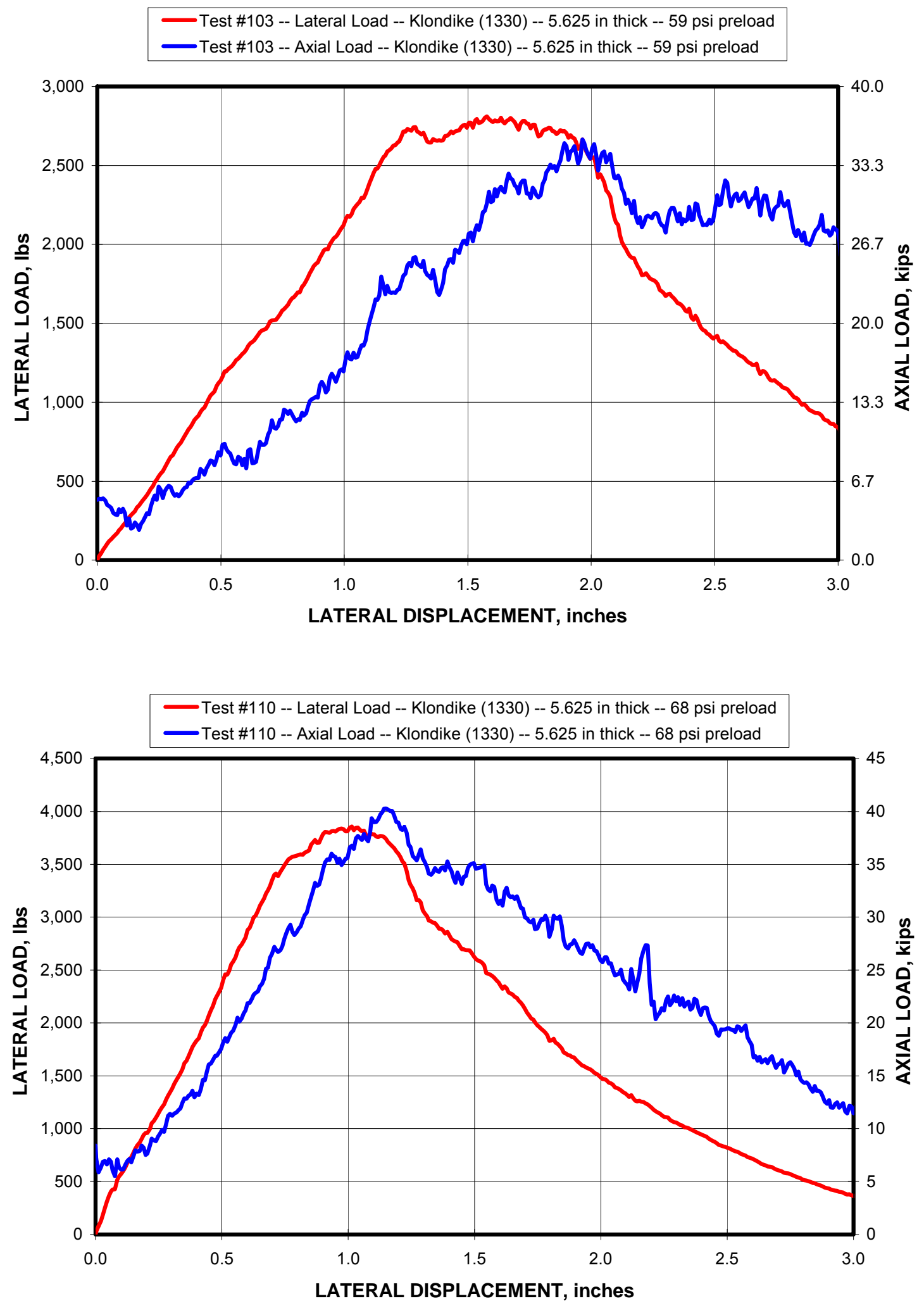

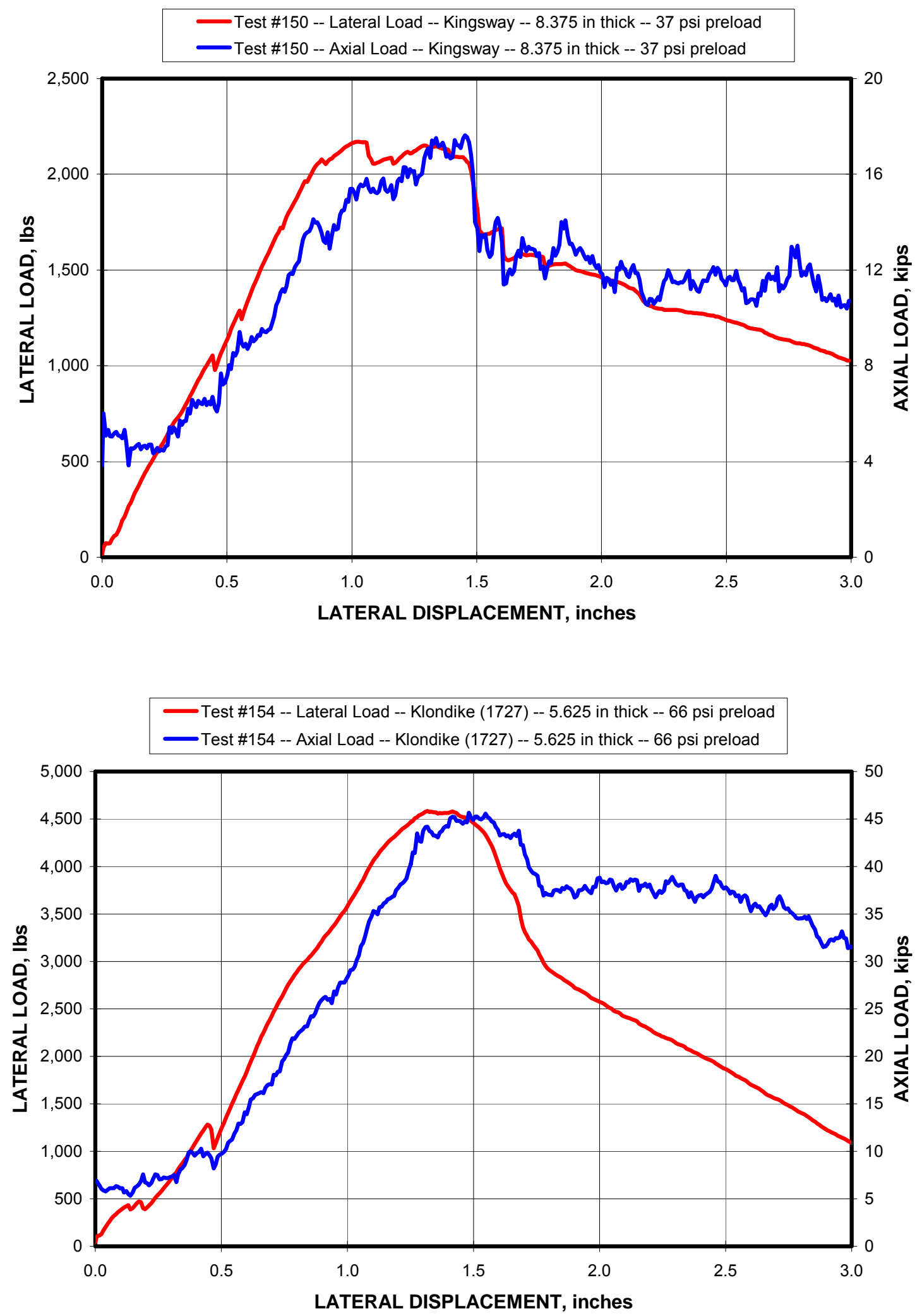


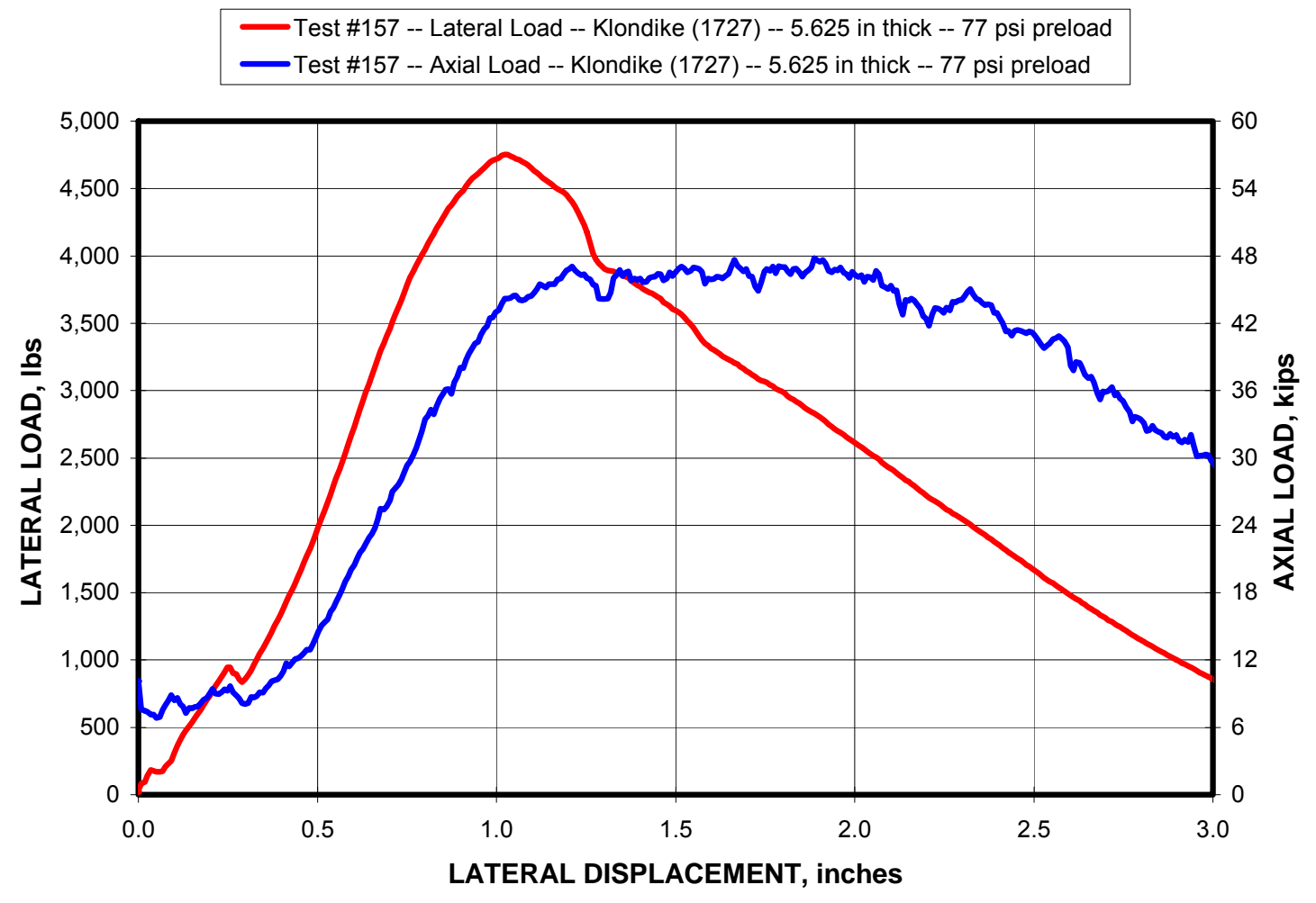




\section{APPENDIX D - DATA ACQUISITION SYSTEM FOR EVALUATION OF FULL- SCALE TESTING IN THE NIOSH EXPERIMENTAL COAL MINE}

The data acquisition system for the tests of ventilation stoppings in the NIOSH Experimental Coal Mine consisted of four channels, the pressure applied to the wall and three measurements of deflection of the specimen. The signals were amplified, digitized and recorded.

The air pressure applied to the stopping were measured using Sensotec Model Z pressure transducers. These devices were a Wheatstone bridge arrangement of strain gages to convert detected pressure to a proportional output voltage. Four pressure ranges from 0.5 psi to $50 \mathrm{psi}$ were available. The output from the sensor bridge was 1-2 millivolts per volt of excitation for the 0.5 psi unit and nominally 3.0 millivolts per volt of excitation or the others. The deflection of the stopping wall was measured at three points along the center vertical axis. The sensors were spring tensioned wire pull transducers, calibrated to 10 inches maximum displacement. The output voltage was proportional to the linear extension of the wire from the transducer mount. This voltage was measured at the center tap of a precision potentiometer with a constant current supply. The sensors were mounted on a vertical rail in close proximity to the surface of the stopping wall. The middle point was halfway between the roof and floor, and the quarter points were halfway between the middle height point and the roof or floor. The wire pull was attached to the stopping in a manner to prevent destruction of the sensors when the wall fails.

The conversion of physical measurement parameters to output voltage was accomplished using Honeywell Accudata Model 218 Bridge Amplifiers. These units were high precision signal conditioning instruments that provide a constant current supply and a variable gain amplifier. Optional settings permit the connection to many different types of sensors. The Wheatstone bridge and three-wire potentiometer configurations were utilized for these tests. Active filters eliminated noise from the output signal. Configuration of the amplifier for the sensor type, selection of the supply voltage to the transducer and the adjustment of the gain of the amplifier ensured the accuracy of the sensor measurements. The channel amplifier converted the final output 
for each transducer to a zero to a ten-volt full-scale value that was connected to the digital data acquisition system.

The output from each amplified sensor channel was converted to a digital value utilizing a National Instruments DAQPad-6020E. This device accepts the signal from the amplifier, converts the measured voltage to a digital value and transmits the digital signal through a Universal Serial Bus port (USB) to a laptop computer for recording. Eight differential inputs were available on the DAQPad. These inputs can also be utilized as sixteen single-ended channels. The differential mode provided improved accuracy of the measured voltage by rejecting common mode voltages induced on the signal lines by nearby electrical equipment. The range of each input channel was set using software on the laptop computer. The DAQPad contains a 12-bit analog-to-digital converter (ADC). The zero to ten-volt full-scale signal from the amplifier was converted to a digital value between 0 and 4,096 bits. This provided a measurement resolution of 2.44 millivolts per bit or better than 0.25 pct.

The DAQPad-6020E was controlled and data was transferred to a Dell Latitude laptop computer running National Instruments VI Logger software under the Microsoft Windows 2000 operating system. The Logger software controls communication with the DAQPad across the USB. The sampling rate, channel input ranges and zero offset values were all selectable in the software. The digital values can be stored representing the measured voltage or converted to engineering units with programmable equations. The data was displayed in real time and stored on the hard disk. Test data files were then converted to Microsoft Excel Workbook format for further processing and analysis. 